\title{
Biodiversity and Distribution of Benthic Foraminifera in Harrington Sound, Bermuda: The Effects of Physical and Geochemical Factors on Dominant Taxa
}

Nam Le

Follow this and additional works at: https://digitalcommons.colby.edu/honorstheses

Part of the Applied Statistics Commons, Categorical Data Analysis Commons, Climate Commons, Geochemistry Commons, Geology Commons, Multivariate Analysis Commons, Paleobiology Commons, Paleontology Commons, Probability Commons, and the Statistical Models Commons

Colby College theses are protected by copyright. They may be viewed or downloaded from this site for the purposes of research and scholarship. Reproduction or distribution for commercial purposes is prohibited without written permission of the author.

\section{Recommended Citation}

Le, Nam, "Biodiversity and Distribution of Benthic Foraminifera in Harrington Sound, Bermuda: The Effects of Physical and Geochemical Factors on Dominant Taxa" (2019). Honors Theses. Paper 920.

https://digitalcommons.colby.edu/honorstheses/920

This Honors Thesis (Open Access) is brought to you for free and open access by the Student Research at Digital Commons @ Colby. It has been accepted for inclusion in Honors Theses by an authorized administrator of Digital Commons@ Colby. 


\title{
BIODIVERSITY AND DISTRIBUTION OF BENTHIC FORAMINIFERA IN HARRINGTON SOUND, BERMUDA: THE EFFECTS OF PHYSICAL AND GEOCHEMICAL FACTORS ON DOMINANT TAXA
}

\section{Nam Le}

\begin{abstract}
A thesis submitted to the Faculty of the Department of Geology of Colby College in fulfillment of the requirements for Honors in Geology
\end{abstract}

Waterville, Maine

May 2019 


\section{BIODIVERSITY AND DISTRIBUTION OF BENTHIC FORAMINIFERA IN HARRINGTON SOUND, BERMUDA: THE EFFECTS OF PHYSICAL AND GEOCHEMICAL FACTORS ON DOMINANT TAXA}

Except where reference is made to the work of others, the work described in this thesis is my own or was completed in collaboration with my advisory committee

Nam Le

Certificate of Approval

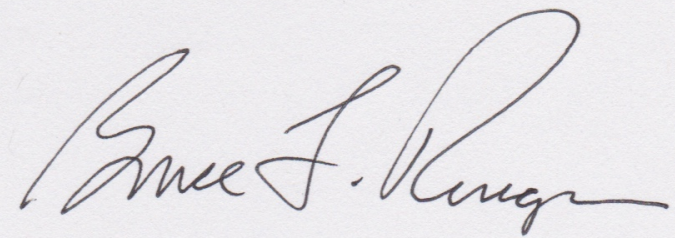

Dr. Bruce F. Rueger

Visiting Assistant Professor of Geology Colby College<smiles>CCCCOCC(C)(C)C(C)(C)C</smiles>

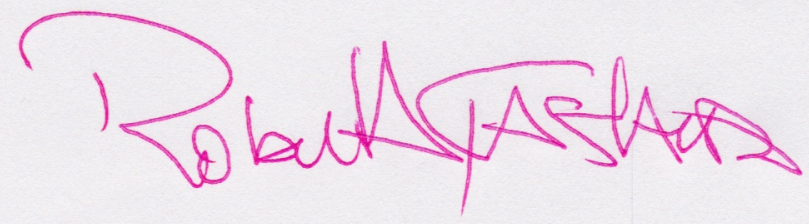

Dr. Robert A. Gastaldo Whipple-Coddington Professor of Geology Colby College

Dr. Struan R. Smith

Curator of the Natural History Museum Bermuda Aquarium Museum and Zoo 


\section{ABSTRACT}

Harrington Sound, Bermuda, is a nearly enclosed lagoon acting as a subtropical/tropical, carbonate-rich basin in which carbonate sediments, reef patches, and carbonate-producing organisms accumulate. Here, one of the most important calcareous groups is the Foraminifera. Analyses of common benthic orders, including miliolids (Quinqueloculina and Triloculina spp.) and rotaliids (Homotrema rubrum, Elphidium spp., and Ammonia beccarii), are essential in understanding past and present environmental conditions affecting the island's coastal environment. These taxa have been studied previously; however, factors explaining their individual patterns of abundance in the Sound are not well detailed. The goal of this study is to understand foraminiferal assemblage trends by assessing parameters including depth, test-size, temperature, salinity, dissolved oxygen, $\mathrm{pH}$, and variable interactions. The results of this study can serve as a baseline model for Neogene carbonate lagoons that accumulated Foraminifera under different climate conditions.

Benthic sediment samples were collected at depths ranging from $3 \mathrm{~m}$ to $24 \mathrm{~m}$ using an Ekman dredge, while water-quality data were collected using an In-Situ SMAR Troll MP probe. Due to the limited test-size of these taxa, four size fractions were examined $(1 / 2+\mathrm{mm}, 1 / 4-1 / 2$ $\mathrm{mm}, 1 / 8-1 / 4 \mathrm{~mm}, 1 / 16-1 / 8 \mathrm{~mm})$. Dead-test and fragment counts were performed after randomizing sample selections with a sediment splitter. Using RStudio and ArcMap, basic, advanced statistical, and geospatial analyses were conducted on each taxon (segregated by test and fragment size) to examine depth-profile and spatial relationships between count data, physical, and geochemical factors.

Results from multiple quantile regression, nonparametric MANOVA, and geospatial modeling show that each taxon, under identical depth and spatial constraints, can be accounted for by different functional combinations of variables. In testing multivariate response and 
factorial influence, initial relative effect estimations and subset algorithms from nonparametric MANOVA effectively quantify abundance patterns. Spatial maps of all test-size fractions of Quinqueloculina \& Triloculina spp. showed that these species were concentrated in deeper and colder regions in Harrington Sound, which disagreed with previously reported studies. Fragment counts of $H$. rubrum increased in shallower and warmer regions, with lower salinity, higher dissolved oxygen, and higher pH. Both Elphidium spp. and A. beccarii test counts were found be concentrated in areas with high salinity, although there were several anomalies across test-size fractions for both taxa.

Although there was a limited number of sampling points and measurements, the study's statistical methods proved to be effective in modeling the distribution of test-size concentrations within a complex system. Thus, as one of the few pioneering studies to cumulatively use predictive, multivariate, and geospatial models, this study urges future research studies to push beyond conventional, quantitative methodological boundaries regarding similar inquiries. 


\section{ACKNOWLEDGEMENTS}

I would like to acknowledge support from the Department of Geology endowment funds, specifically the Ed and Whitney Selover Family \& Barrett T. Dixon Geology Research and Internship Funds. I appreciate the collaboration and assistance of: Roger Hollis, Straun Smith, Trevor Rawson, and Nigel Pollard, Bermuda Aquarium Museum and Zoo, Bermuda, for equipment and sampling assistance; Merrill Read, Marcus Jones, David Miller, and Jill Clinton, Colby College, for field assistance; Dr. Robert Gastaldo, Colby College, for use of laboratory facilities; Dr. Whitney King, Colby College, for use of water chemistry equipment; Ralph J. Bunche Scholars, research grant funding; Buck Environment and Climate Change Lab, for funding our research trip to Bermuda. 


\section{INTRODUCTION}

Harrington Sound represents a $4.79 \mathrm{~km}^{2}$ nearly enclosed, marine lagoon located in the main island of Bermuda. This basin acts as a subtropical/tropical, carbonate-rich catchment in which carbonate sediments, deep Oculina spp. beds, and skeletal remains of other carbonateproducing organisms accumulate (Morris et al., 1977; Wefer, 1980). Its formation was thought to have occurred much later than the islands' origin, which began during the late Pleistocene. During the long-term growth of the island, a cap of eolian limestone-dune sediments began to develop approximately 1 to $1.6 \mathrm{Ma}$ ago and were highly susceptible to fresh-water dissolution in response to low stands of sea-level. When sea level was lower, fresh-water percolated through the sediments, resulting in dissolution in the limestone cap; when sea level rose, fresh-saltwater mixing further exacerbated dissolution (Vacher and Quinn, 2004). However, as marine biota colonized Bermuda, bioerosion became the islands' main driver of inshore expansion and development (Neumann, 1965).

One of the most important nearshore biological group in Bermuda is the Foraminifera, which are calcareous, amoeboid protists that produce calcite shells (tests). These protists grow in response to the prevailing physical/chemical conditions and are essential in understanding past and present environments. The study of Foraminifera goes back to the $5^{\text {th }}$ Century BC when the Greek Historian Herodotus noted that the limestones in the Egyptian pyramids possessed large benthic forams (Boudagher-Fadel, 2015). The group possesses a geological record ranging from Early Cambrian to present day; however, due to Bermuda's short geologic history, ranging from Pleistocene to Recent, Bermudan foraminiferal records are primarily of modern origin (Jauvaux and Scott, 2004).

Foraminifera are classified based on their life habit as benthic or planktonic, where benthic forams live on the sediment floor while planktonic forams float in the water column. 
Two benthic groups are found in Harrington Sound and make up the majority of the skeletal remains in the bottom sediment: Quinqueloculina \& Triloculina spp. (Miliolids), Homotrema rubrum (Rotaliid), Elphidium spp. (Rotaliids), and Ammonia beccarii (Rotaliid). These taxa have been identified in bottom sediments of Harrington Sound, but their ecology in Bermuda has not been fully explored. Therefore, this study will reference ecological parameters of the four taxa known from other Atlantic sites to infer their distribution in Harrington Sound.

Quinqueloculina \& Triloculina spp. are benthic genera in the Miliolid order, and characterized by imperforate, porcellanous calcite tests. They are distinctly coiled, with chambers alternating regularly in three planes (Triloculina) of coiling 120 degrees apart and five planes (Quinqueloculina) of coiling 72 degrees apart (Loeblich and Tappan, 1964). Many foram workers believe that these taxa appear in mass abundances at certain times of the year in other subtropical environments and end their life cycle after a few weeks to three months, where the number of living specimens drop from $100 \%$ to $5 \%$. Ecologically, miliolid foraminifera are generally more concentrated in high Mg-calcitic environments, for example in karst platforms and warmer climates (Neumann, 1965).

Homotrema rubrum is a benthic genus in the Order Rotaliid, and characterized by an encrusting, high Mg-calcite test that grows and develops in cracks, crevices, and overhanging shaded parts of reefs. The life habit of this genus is different from the other benthic genera because they are sessile and known to attach to corals and algae (Blackmon and Todd, 1959). Because they secrete mineralized skeletons, $H$. rubrum is very well-preserved in the fossil record, and are frequently used as a paleoproxy for past ecological or physical disturbances (Taylor and Wilson, 2003). The abundance of this taxon is strongly dependent on environmental conditions; however, their lifespan typically ranges from one to two years. Their unusual 
coloration is responsible for the famous Bermudan pink sand. It is difficult to collect complete tests of $H$. rubrum; hence, most analyses are performed on fragments, the distribution of which is highly dependent on high-wave energy environments (Machado and Moraes, 2002).

Elphidium spp. is a benthic genus in the Order Rotaliid, characterized by spiral-shaped, calcite or silica translucent tests, composition of which varies across order. These vary from biconvex test symmetry, multilocular chamber arrangements, and perforated structures, with a chiseled surface comprised of v-shaped chambers. Foraminifera scholars have found this genus to be very abundant across geographic localities and temperature ranges (Schweizer et al., 2011). Their lifespan tends to significantly vary by species and size; small individuals, less than one millimeter in size, generally live up to three months whereas those larger than one millimeter can live up to a year. Small benthic forams like Elphidium spp. are generally abundant in shallow marine environments with fluxes in salinity that mimic coastal/oceanic conditions (Roozpeykar and Moghaddam, 2015).

Ammonia beccarii is a benthic species in the Order Rotaliid with a biconvex, threedimensional, spiral calcite test. This species possesses ten angulated chambers near the umbilical side, a common chamber structure in rotaliids. Due to its geographically widespread abundance, Ammonia beccarii has been useful in understanding changes in foram paleoecology on a global scale. This is because the taxon is used to correlate microhabitat shifts with environmental change (Debenay et al., 1998). Like Quinqueloculina \& Triloculina spp., the taxon is believed to appear in mass abundances during selective parts of the year and die off after a few weeks to three months. Like Elphidium spp., Ammonia beccarii generally thrives in shallow marine environments with coastal/oceanic, salinity conditions; however, the taxon also is believed to be 
epiphytic on corals (Debenay et al., 1998) and tolerant of eutrophic conditions (Roozpeykar and Moghaddam, 2015).

\section{BACKGROUND}

Previous studies on Harrington Sound have primarily focused on its hydrology and cave geology, with few scholars having examined the paleoecology of Foraminifera. Prior to 2004, the only studies examining Harrington Sound's forams investigated stable isotopes of larger foraminifera in 1980, habitat and reproduction of specific species in 1980, and a core taken just south of Trunk Island in 1981. Wefer and Berger (1980) and Wefer et al. (1981) examined oxygen and carbon isotopes to understand differences in life histories and temperature changes over time. Lutze and Wefer (1980) investigated the habitat and asexual production stages of the larger foraminifera Cyclorbiculina compressa, understanding maturity life cycles both in the field and in the laboratory. Erkenkeuser et al. (1981) studied the environmental changes over the last 9000 years using benthic taxa. Over two decades later, Javaux and Scott (2004) attempted to catalogue most modern benthic foraminifera in the Bermuda Islands, resulting in the recognition of 300 species. Using this comprehensive catalogue, the initial stages of the current project were dedicated to correctly identifying and matching the forams in samples to their catalogue; this was the crucial step in identifying the dominant taxa. Using the foraminiferal data from these two studies in conjunction with preliminary data, this project focuses on identifying the multivariate relationships between environmental factors and test/fragment counts found in bottom sediments across a transect of Harrington Sound. Furthermore, this study aspires to explore innovative computational methods that analyze non-normal data and perform non-metric analysis in micropaleontology. 


\section{LOCALITY}

Harrington Sound is the largest enclosed body of marine water in Bermuda. It is located in east central Bermuda and is almost entirely surrounded by the Main Island. The Sound has one direct surface opening to the Atlantic Ocean through Flatt's Inlet, a 26.4-meter-wide channel situated almost exactly between two major municipalities, Hamilton and St. Georges. Water exchange into and out of the basin is also known to occur through underwater cave passageways, specifically in the Walsingham isthmus between the Sound and Castle Harbor. Morris et al. (1977) found that Harrington Sound acts like a pump, and is not in equilibrium, with a net inflow of $63 \%$ net through the inlet and a $35 \%$ net outflow through the island/cave passageways. This indicates that although efficient exchange with outside waters is limited, it is enough to sustain fully marine-water properties. Furthermore, because the Sound has limited connection to the Atlantic Ocean, sedimentation processes are limited. Neumann (1965) describes the Sound as the closest representation to a small-scale, closed system with minimal sediment exchange with outside sources. Therefore, Harrington Sound is known as a carbonate sediment trap, where the basin provides and collects sediment within itself (Neumann, 1965). The sediment composition of the Bermudan limestones and the inshore ocean floor is primarily calcareous skeletons, with a high proportion of Foraminifera tests and mollusk shells, and fragments that are often subjected to cementation, recrystallization, and mineral replacement (Friedman, 1964).

\section{HYPOTHESES}

The goal of this study is to understand assemblage trends in the four foraminiferal taxonomic groups by assessing the influence of environmental parameters including depth, temperature, dissolved oxygen, salinity, and $\mathrm{pH}$. There are two sets of questions being tested. The first concerns whether a relationship exists between functional combinations and 
interactions of environmental variables and Foraminifera test and fragment counts. If so, do they vary among the taxa? How do these results compare to data from previous literature? The second is whether there are geospatial relationships and factors we cannot constrain through data modeling. If so, what types of complex factors and relationships can we identify through spatial mapping? How do these results compare to data from previous literature?

The purpose of the first set of questions is to assess if physical and geochemical variables affect the changes in test and fragment counts and, if so, in what ways. To answer this first set of hypotheses, we conducted regression models, variance analyses, and ordination to numerically and categorically identify factors that are strongly associated with test and fragment counts. The second set of hypotheses calls into question the relationships, physical and geochemical factors, and errors that could not be answered in the first set of hypotheses. This is a vital component of the project because profile models are two-dimensional analyses that do not account for spatial and temporal distributions or trends. Hence, geospatial maps will provide the three-dimensional analysis for this project. Furthermore, the results of this study can serve as a baseline model for Paleogene to Neogene carbonate lagoons that accumulated Foraminifera under different climate conditions.

\section{METHODOLOGY}

\section{Sample Collection, Processing, and Analysis}

Bottom-sediment and water samples for chemical analysis were collected from 9 sample sites using an Ekman dredge and an In-Situ Inc. SMAR Troll MP probe, respectively. At every sampling site, the collection depth, water temperature, salinity, rugged dissolved oxygen (RDO), redox potential (ORP), and $\mathrm{pH}$ were recorded. While the sediment-grab sampler was dropped into the water, the water quality probe was lowered to the sediment-water interface at a rate of 
one meter per minute. The sampling procedure was changed and slowed to half a meter per minute after the probe entered the thermocline because it was necessary to accurately capture the distinct drop in temperature. Water chemistry data were saved in .csv file format for importation and analysis using Rstudio and Excel. The grab samples were left in the sun for 24 hours to settle and separate excess water from the sediment. Subsequently, sediment samples were dried and packaged for return to Colby College. The grab samples were further processed at Colby College where they were sieved through $<1 \mathrm{~mm},<0.500 \mathrm{~mm},<0.250 \mathrm{~mm},<0.125 \mathrm{~mm}$, and $<0.063 \mathrm{~mm}$ size fractions to remove excess fine-grained material. These samples were left to dry for 24 hours prior to microscopic analysis. During January 2019, we returned to Harrington Sound, Bermuda, to collect additional water-chemistry data to understand seasonal variations in aqueous geochemical properties; this was the conclusion to the field sampling component of the study. Sample Site Distribution

There are four major geologic features within the perimeter of Harrington Sound:

Patton's Basin, North Basin, Middle Ridge, and South Basin (Figure 1). Patton's Basin is located directly southwest of Trunk Island and directly northwest of Devil's Hole, with a depth range of 17-24 meters; North Basin is directly northeast of Trunk Island and directly west of Hall's Island, with a depth range of 15-20 meters; Middle Ridge is directly south of North Basin and directly southeast of Trunk Island, with a depth range of 10-15 meters; South Basin is directly southeast of Middle Ridge, directly west of Shark Hole, and northeast of Devil's Hole, with a depth range of 17-22 meters. According to the modified version of Bates (2017)'s bathymetric map, Harrington Sound's profile is highly complex, and it was important to account for steep depth-and-slope changes during the sample-collection process. As seen in Figure 2, sample sites 1, 3, and 5 were around Trunk Island, site 4 was east of Flatt's Inlet, site 6 was east of Green Bay Cave, site 7 was collected in the northern section of Patton's Basin, site 10 was collected in the 
southern section of Patton's Basin/neighboring the northern section of Devil's Hole, site 8 was collected in the northern section of South Basin/neighboring the southern section of Middle Ridge, and site 2 was collected offshore of the old post office and in Middle Ridge. Due to mechanical failures and collection difficulties, site 9 was omitted, lacking samples in North Basin. Thus, the sampling transect begins at site 4 in the southwest corner towards site 2 in the northeast (Figure 2). To address the uneven sampling distribution, spatial models were produced with caution and results were interpreted solely in the area possible for interpolation and regression smoothing.

Water-chemistry measurements around major cave openings and channel inlets were taken as a countermeasure for constraining freshwater/ocean water influx from cave systems and Flatt's Inlet. Samples were collected during June 2018 and January 2019 to capture seasonal variations in temperature, rugged dissolved oxygen, salinity, and $\mathrm{pH}$. During the summer, the water was measured at four locations during outgoing tide: (1) Green Bay Cave; (2) Flatt's Village manmade cave; (3) Shark's Hole; (4) Flatt's Inlet (Figure 3A). During the winter, an additional site by the Old Post Office was added to account for the major cave systems in Hamilton Parish on the eastern end of Harrington Sound (Figure 3B). At each site, two measurements were acquired, one at the direct cave opening and the other at approximately $6 \mathrm{~m}$ away, ultimately to record any evidence of geochemical localization effects. However, after preliminary analysis of the summer geochemical data, it was observed that these samples needed to be retaken during different tide cycles (incoming, outgoing, slack) in the winter to account for strong tidal currents that might have affected the values (Figure 3B). 


\section{Count Analysis}

The foraminiferal context of each sample was assessed using a 100-count analysis. This count total was selected because under a $5 \mathrm{~g}$ sample size, 100 counts of four taxa are statistically likely to represent the sample; a larger sample size of $10 \mathrm{~g}$ would require 200 or 300 counts (de Vernal et al., 1996). For each sample site, four 100-count analyses were performed to assess counts for each test/fragment size interval. The size-fractions were distinguished under the following four categories: (1) coarse (> 1/2 mm); (2) medium (1/4 - 1/2 mm); (3) fine (1/8 - 1/4 $\mathrm{mm})$; (4) very fine $(1 / 16-1 / 8 \mathrm{~mm})$. The counts were visually completed using an Olympus SZX12 stereoscope and conducted using a Gemmy Industrial Corp. Hand Tally Blood Cell Differential Counter to manually count the different four taxa: Quinqueloculina \& Triloculina spp., Homotrema rubrum fragments, Elphidium spp. and Ammonia beccarii. To minimize error rate and maintain count consistency, two trials of 100-counts for each site were performed. This is a necessary check because it allows for quantile regression models to estimate the $50^{\text {th }}$ quantile between the two trials' residual differences.

\section{Computational Analysis}

In preparation for computational analysis, 100-count data were merged with water chemistry data to produce a comprehensive dataset and was saved in a flat file (.csv) format to input and analyze in Rstudio and ArcGIS. Once the dataset was complete and in a flat-file format, statistical modeling, numerical optimization, and geospatial mapping were undertaken to visualize, model, and test the quantitative data across three components: Normality Testing, Profile Analysis, and Geospatial Analysis. Prior to conducting any form of statistical test or modeling tool, the distribution of the data needs to be fully understood and visualized. Parametric statistical analyses require that all data used are Gaussian in distribution. In contrast, 
non-parametric statistical analyses do not rely on theoretical distributions to conduct any test or modeling tool. To fulfill this step, Shapiro-Wilk Normality tests were run to determine whether each data set was normally distributed and, if so, could be analyzed using linear regression modeling, MANOVA, and principal component analysis (PCA). The standard null and alternative hypotheses are defined as following: $\mathrm{H}_{\mathrm{o}}$ - Population is normally distributed; $\mathrm{H}_{\mathrm{a}}-$ Population is not normally distributed. Under these assumptions, this study's normality procedure states that if at least $3 / 4^{\text {th }}$ of all variables fail to reject $\mathrm{H}_{\mathrm{o}}$, parametric statistics are appropriate. And if at least $1 / 4^{\text {th }}$ of all variables reject $\mathrm{H}_{0}$, non-parametric statistics can be used. For more details related to the theorems and notation of the Shapiro-Wilk Test, refer to Shapiro and Wilk (1965).

Geoscientists and paleontologists have dealt with this analytical issue for decades, where identifying the proper tools and functions to assess non-normal data have been a recurring problem. Fortunately, modern ecologists and paleontologists have found that quantile regression is an effective alternative to multiple linear regression, a technique that previously was utilized mainly in community ecology and econometrics. Hence, statisticians computationally modified many statistical packages and functions to perform the non-parametric functions (Cade and Noon, 2003). The adjusted profile-analytical techniques employed in the current study are quantile regression, non-parametric MANOVA (NPMANOVA), non-metric multidimensional scaling (nMDS), and analysis of similarity (ANOSIM). Using quantile regression and NPMANOVA, functional combinations of environmental factors can be examined to model which variables are most closely associated with test/fragment counts.

Quantile regression is a technique that estimates the conditional median or other quantiles of the response variable unlike linear regression, which estimates the conditional mean of the 
response variable. This is an important adjustment because conditional medians indicate the exact middle quantile of the data and are more robust to outliers than conditional means; the results of which illustrate a regression model that is not influenced by extreme values.

Often, geoscientists will use the classical least squares regression (CLR), under log, square root, and box-cox transformations, as the conventional approach to estimating unconditional mean parameters to estimate conditional mean functions. Here, the objective is to formulate an optimization problem encompassing both components; the generic CLR model is denoted as the following:

$$
y_{\mathrm{i}}=x_{\mathrm{i}} \beta+u_{\mathrm{i}} \quad(\text { Equation } 1)
$$

where $y_{\mathrm{i}}$ represents the response, $x_{\mathrm{i}} \beta$ represents the independent variable(s) and its coefficient, and $u_{\mathrm{i}}$ represents the y-intercept (Koenker and Hallock, 2001)

In contrast, quantile regression optimizes a piecewise linear objective function in the residual values to estimate conditional quantile functions; the generic $\mathrm{QR}$ model is denoted as the following:

$$
\varepsilon_{\mathrm{i}}(\tau)=x_{\mathrm{i}} \beta(\tau) \quad(\text { Equation } 2)
$$

where $\varepsilon_{i}(\tau)$ represents the response that is not identically distributed and is subjected to a specific quantile $(\tau)$ i.e. $25^{\text {th }}, 50^{\text {th }}, 75^{\text {th }}, x_{\mathrm{i}} \beta$ represents the explanatory variable and its coefficient, and the $\tau$ value represents the conditional quantile the model is estimating (Koenker, 2018).

In this current project, the $50^{\text {th }}$ quantile will be used to estimate the conditional median of the response variable, test and fragment counts. This is also known as median regression, which minimizes the sum of absolute residuals when each sampling location has two trials with two 
different sets of values (i.e., for each geochemical and physical parameter, there are two sets of corresponding count values).

The most important advantage to using quantile regression over linear regression is its robustness to distributional assumptions, which are always an important guideline in statistics. The optimized estimations and associated inference components are inherently distribution-free in character because quantile estimation is influenced by local behavior of the response variable's conditional distribution in the quantile region (Koenker, 2018). Despite these unique qualities, the model interpretation slightly differs from an CLR, specifically with coefficients. For each explanatory variable, the coefficient estimates are interpreted as the quantitative impact of a one-unit change of the variable on a specific quantile of the response variable, while holding other covariates fixed (Koenker and Hallock, 2001). In the predictive modeling silos of this study, each model uses combinations and interactions of depth, temperature, rugged dissolved oxygen, salinity, and $\mathrm{pH}$ to predict the median estimate variability of foraminiferal test/fragment counts of one taxon under one test/fragment-size fraction. Variable interaction indicates that the significance of one explanatory variable towards test count is dependent on the variability of another explanatory variable. Here, it is important to note that the predictive models do not account for multiple response variables, which are the different taxa coexisting in Harrington Sound. Therefore, the technique's observable limitation is its lack of scope for response covariance.

Similarly, NPMANOVA is the non-parametric alternative to Multivariate Analysis of Variance. This technique is used to determine the independent variables' effect on response variables, the relationships between the independent variables, and the relationships between the response variables. Unlike traditional MANOVA, NPMANOVA performs permutation tests on 
the original results to guarantee repeated randomization. The results of additional randomization assess the extent of statistical significance of the non-permuted results.

In a non-parametric multivariate model, there are two sets of hypotheses that are tested: Global Statistical Hypotheses (GSH) and Subset Algorithms (SA) (Burchett et al., 2017). GSHs are typically the following: Are the $a$ samples from the same population? Do the $a$ explanatory parameters have the same effect? In conventional notation, $\mathrm{H}_{\mathrm{o}}$ states that $\mathrm{F}_{1}=\ldots=\mathrm{F}_{\mathrm{a}}$, while $\mathrm{H}_{\mathrm{a}}$ states that at least one F is not the same. To run this model, GSHs require eight tests: four types, each under F approximation and permutation. The four tests used are ANOVA-type test, McKeon approximation for the Lawley-Hotelling Test, Muller approximation for the BartlettNanda-Pillai Test, and Wilks Lambda (Burchett et al., 2017). In cases with high-dimensional data, Wilks Lambda cannot be calculated and is replaced by the ANOVA-type test. Up until this point, NPMANOVA is the same as its parametric counterpart, but the next step introduces sample-size constraints using permutation and randomization tests. If sample size is less than 10 , the permutation test is run; if sample size is between 10 and 30, the randomization test is run with 10,000 randomly permutated tests; if sample size is greater than 30 , the F approximation is used (Burchett et al., 2017). Because there are nine sample sites, duplicated with two count trials each, the study's sample size is 18 . Hence, all NPMANOVA tests will undergo a randomization test with 10,000 permutations. Furthermore, if $\mathrm{H}_{\mathrm{o}}$ is rejected, the next steps are to investigate which environmental parameter(s) and its factor levels contribute to overall effects using SAs. This is a post-hoc test.

Subset Algorithms are two-stage, partition-hypotheses procedures that follow a global test rejection, where $a$ response variables are tested for difference and $a$ factor levels in each explanatory variable are tested for difference (Burchett et al., 2017). In context, this multi- 
faceted procedure asks two sets of questions: 1) Are there count differences among Quinqueloculina \& Triloculina spp., Homotrema rubrum, Elphidium spp., and Ammonia beccarii? At different test-size fractions? Which ones are different? 2) Are there differences among depths (Shallow Sandy Zone, Oculina Zone, Subthermocline Zone), temperature (Mixed Layer, Thermocline, Subthermocline), rugged dissolved oxygen (67-100\% Saturation, 44-67\% Saturation, 22-44\% Saturation, 0-22\% Saturation), salinity (Mesohaline, Polyhaline, Euhaline), and $\mathrm{pH}(8.0-8.2,7.8-8.0,7.6-7.8)$ ? At different test-size fractions? Which ones are different? And by testing these questions, one can comprehend the power of a MANOVA model under non-parametric assumptions.

In addition to the benefits of a multivariate model without the strict testing assumptions, NPMANOVA offers a numerical calculation called the Empirical Non-Parametric Relative Treatment Effects (RTE). These are numerical measures quantifying the tendencies of the data in probabilistic values. The interpretation of a RTE value is: the probability that a randomly chosen subject from treatment $k$ displays a higher response than a subject randomly chosen from any other treatment groups, including treatment $k$ (Burchett et al., 2017). In other words, it is the probability that a response variable is more likely to occur in a specific treatment group than in any other treatment group. For example, let's assume that the global hypothesis is rejected, and subset algorithms are statistically significant for the medium test-size fraction for all four taxa vs depth. If the RTE value in the medium size-fraction of Ammonia beccarii is 0.875 for the Oculina Zone, the proper interpretation is stated as following: the probability that there are more medium-fraction sized Ammonia beccarii test counts in the Oculina Zone compared to other depth zones is $87.5 \%$. Furthermore, this calculation is the missing piece in conventional 
multivariate models, allowing one to quantify physical and geochemical tendencies in this study's data.

An additional tool that geoscientists often use in data analysis is ordination, specifically Non-Metric Multidimensional Scaling (nMDS) is the process of low-dimensional mapping of dissimilarities and a non-parametric ordination technique (Legendre and Legendre, 1998). In a way, using nMDS, the regression and multivariate analyses are exported to a low-dimensional, ordinal space to further explore these relationships in a simpler, simulated environment. Unlike other ordination methods, which are analytical and result in a single set of solutions for each dataset, nMDS is a numerically optimizing technique that searches for a suitable solution and stops computation when that objective is fulfilled (Legendre and Legendre, 1998). This technique makes very few, if any, assumptions about linear relationships or any other parametric conditions, allowing analytical compatibility for non-normal datasets.

Unlike real-space analysis, which requires one comprehensive matrix with explanatory and response variables, nMDS in ordinal space requires two separate data matrices for explanatory and response variables that undergo pairwise distance calculations to produce symmetrical data matrices. In this study, the two data matrices are taxonomic (Quinqueloculina \& Triloculina spp., Homotrema rubrum, Elphidium spp., Ammonia beccarii) and environmental data (depth, temperature, RDO, salinity, $\mathrm{pH}$ ). Furthermore, these distances are regressed against the original matrix and predicted ordination distances for each sample pair (Legendre and Legendre, 1998). The resulting goodness-of-fit regression model is defined as stress, which abides by several modified functions, one of which is the following formula:

$$
\text { Stress }(1)=\left(\sum_{h, i}\left(d_{h i}-\hat{d}_{h i}\right)^{2} / \sum_{h, i} d_{h i}{ }^{2}\right) \quad(\text { Equation 3) }
$$


where $d_{h i}$ is the ordinated distance between samples $\mathrm{h}$ and $\mathrm{i}$, and $\hat{d}_{h i}$ is the predicted distance from regression (Legendre and Legendre, 1998).

Computationally, the objective of this model is to test and retest the distance matrix and regression to ultimately reach a specified tolerance value or optimally low values of stress (Oksanen, 2015).

After performing nMDS and calculating optimal stress values, the newly-scaled data matrix needs to be analyzed using Analysis of Similarities (ANOSIM), an ANOVA model for a distance matrix. In other words, unlike ANOVA, which only compares the means between groups in raw data, ANOSIM compares the mean of distances or dissimilarities between and within groups (Warton et al., 2012). In this model, the null and alternative hypotheses are stated as: $\mathrm{H}_{\mathrm{o}}$ - there is no dissimilarity between the groups, $\mathrm{H}_{\mathrm{a}}$ - there is dissimilarity between the groups (Clarke, 1993). Within this function, the most important value is the ANOSIM R-statistic, which is based on the difference of mean distances between groups and within groups and is the following formula:

$$
R=\left(\mathrm{r}_{B}-\mathrm{r}_{W}\right) /(\mathrm{N}(\mathrm{N}-1) / 4) \quad(\text { Equation } 4)
$$

where $\mathrm{r}_{B}$ represents means between groups and $\mathrm{r}_{W}$ represents mean distances within groups (Clarke, 1993).

To ensure the strength of the model outputs, we run 999 randomized permutation tests on the distance matrix. From there, we used the R-statistics to discern if there is dissimilarity between or within groups under the following guidelines: 1) R-values close to 1 suggest dissimilarity between groups; 2) R-values close to 0 suggest even distribution between and within groups; and 3) R-values below 0 suggest there is more dissimilarity within groups than between groups 
(Warton et al., 2012). In context, under reduced dimensions, R-values indicate if there is dissimilarity in the mean test/fragment counts among the different treatment groups (i.e., depth zones, RDO levels), and if there is dissimilarity in the mean test/fragment counts in each treatment group. Furthermore, these results help us identify multivariate relationships in reduced dimensions, which are compared to real-space dimensions produced by NPMANOVA.

\section{Geospatial Analysis}

Following the statistical and numerical techniques in profile analysis, we created and used geospatial maps and models to explore spatial and temporal relationships between environmental factors and test/fragment-count results. The two software programs are Rstudio and ArcGIS, where mapping is produced using Additive Quantile Regression Smoothing (AQR) and Triangulated Irregular Networks (TIN). Building on the profile perspective provided by statistical modeling, spatial statistics play a major role in revealing unconstrained factors and variables that might be responsible for data anomalies or outliers, ultimately providing a threedimensional component to the study's computation.

Originally introduced by Koenker and Mizera (2004), AQR smoothing is a nonparametric smoothing tool that uses triogram methods, which are function estimations that use piecewise, bivariate splines on an adaptively-constructed triangulation process (Hansen et al., 1998). AQR requires three components: 1) spatial constraints $x$ and $y$, which, in this case, are latitude and longitude values; 2 ) $\lambda$, the smoothing parameter (0.01 is used); and 3 ) ndum, the number of triangulated artificial vertices (100 is used) (Koenker, 2018). The advantage of AQR is to use quantile regression algorithms to fit non-parametric, bivariate contours, which is not possible to replicate in popular mapping platforms like ArcMap or QMap. After creating contour plots for each environmental parameter, we identified and sketched spatial vectors for each map, 
and oriented these observations on the published depth map from Bates (2017). However, the drawback of this technique is its computational inability to overlay multiple contours to analyze multiple variables in each model. Thus, these findings were extracted from Rstudio and further processed and examined in ArcGIS.

The two functions in ArcMap used in this study are Triangulated Irregular Networks (TIN) and Surface Contour, which collectively produces a multivariate spatial model. I decided to use TIN to create gradient maps for both environmental factors and foraminifera taxa. From there, Surface Contour is employed to stack additional contour layers to the base gradient and has the option to intersect contour lines of both layers together. The last step of geospatial analysis is creating a graduated symbol layer for geochemical and physical properties, such as salinity and $\mathrm{pH}$, attained from Flatt's Inlet and underwater cave passageways. This is a necessary process because it helps us understand and constrain for localized effects (i.e., fresh-water mixing, increased acidity, etc).

Overall, this study requires a large combination of computational modeling because each technique satisfies one part of the full picture. Regression modeling, NPMANOVA, and nMDS provide a profile analysis of the data while geospatial maps account for unexplained factors and errors statistical models miss (spatial and temporal distributions, fresh-water influx from caves, etc.).Thus, from dropping an Ekman dredge and lowering a water quality probe to computing statistical significance, the combined use of field sampling and advanced analytics are necessary to further understand the relationship between the distribution of Foraminifera tests/fragments and Harrington Sound's complicated environmental parameters. 


\section{RESULTS}

Physical and geochemical data distribution along sampling sites

Depth, temperature, salinity, dissolved oxygen, and $\mathrm{pH}$ were recorded at the sedimentwater interface of each sampling site and plotted alongside other sampling sites. Without referring to the spatial distribution of the sites seen in Figure 2, the measured depths illustrated three changes in trend: (1) a recognizable increasing trend moving from Flatts Inlet in the southwest corner all the way to site 10; (2) a decreasing trend from site 10 towards sample 1 along the transect; and (3) a significant increase in depth from site 1 towards Harrington Sound Road (Figure 4A). The overall scatter of different depths is evenly distributed, accounting for the three depth zones: (1) Shallow Sandy Zone (0-10 m); (2) Oculina Zone (10-18 m); (3) Subthermocline Zone (18-25 m). When considering spatial relationships, depths associated with samples 1, 3, and 5 ranged from 4-12 meters, consistent with the depths around Trunk Island; sample 2 was measured at 15 meters depth, consistent with the southern edge of Middle Ridge; sample 4 was measured at 7 meters depth, consistent with depths along Flatt's Village up to Flatt's Inlet; sample 6 was measured at 18 meters depth, accurately capturing the steep slope change at the northeast edge of Patton's Basin; sample 7 was measured at 19 meters depth, consistent with the depth gradient of Patton's Basin; sample 8 was measured at 17 meters depth, accurately depicting the boundary between Middle Ridge and South Basin; and sample 10 was measured at 24 meters depth, consistent with the depth ranges of southern portion of Patton's Basin going towards Devil's Hole (Figure 4A). Thus, based on a map published by Bates (2017), this study's depth measurements were consistent with previous efforts.

Consistent with the depth distribution in Figure 4A, the temperature distribution is correlative with water depth and temperature in an inland body of water. There is a significant drop in temperature around 18-19 meters depth, known as the thermocline (change is seen 
moving from sample 6 to 10 and from 1 to 8 ) (Figure 4B). To characterize the thermocline, water temperature at the sediment-water interface from 0-17 meters depth stays isoclinal at around $28^{\circ} \mathrm{C}$ during the summer; however, there is a significant drop from 28 to $24^{\circ} \mathrm{C}$ from depths of 17-19 meters. And within the vicinity of the deepest spot in the entire Bermuda system, the sediment floor was slightly below $23^{\circ} \mathrm{C}$, a 5 degree drop within a 5-meter depth drop (Figure 4B). When comparing measurements from this study with those taken from Neumann (1965)'s study, the temperature gradient is very similar, if not, identical. The exception is that the thermocline measured in July $1^{\text {st }}, 2018$, was 18 meters versus Neumann's record in 1965, which was approximately 17 meters.

Data complications arose in this study because salinity measurements lacked a discernable trend across sampling locations. Salinity values jump from 3 to 37.5 psu, indicating a drastic change from mesohaline, brackish waters to euhaline, ocean waters (Figure 4C). Thus, unlike Neumann (1965)'s data, which projected a halocline around the same depth as the thermocline, salinity variability in this study is not consistent with previous reports.

Consistent with both depth-temperature distributions, rugged dissolved oxygen experiences a decreasing trend from Flatt's Village to sampling site 10, with a significant drop in value between site 6 and site 10 (Figure 4D). From site 4 to site 10, dissolved oxygen content ranged from 0 to $5.5 \mathrm{mg} / \mathrm{L}$, illustrating a drastic drop in dissolved oxygen towards anoxic conditions (Figure 4D). From site 5 towards the northeast, the dissolved oxygen content ranged from 5.5 to $7.2 \mathrm{mg} / \mathrm{L}$, showing little variability and healthy levels of dissolved oxygen in Harrington Sound.

With less visual variability but similar trajectory to depth, temperature, dissolved oxygen, and $\mathrm{pH}$ show a decreasing trend from site 4 towards site 10 , with significant variability between 
site 6 and site 10 (Figure 4E). Unlike other variables, $\mathrm{pH}$ works in a logarithmic scale and, thus, a change from 8.2 (normal ocean waters) to 7.6 (southern Patton's Basin) equates to approximately $151 \%$ increase in aqueous acidity. The $\mathrm{pH}$ ranged from 8.1 to 7.7 , which equals an approximate $100 \%$ increase in aqueous acidity and, therefore, illustrating significant variability in $\mathrm{pH}$ across Harrington Sound (Figure 4E).

Physical and geochemical data distribution along cave openings and Flatt's Inlet

Physical and geochemical parameters at major cave openings and Flatt's Inlet were recorded as variability constraints. In the summer samples, recorded on July $1^{\text {st }}, 2018$, during outgoing tide, depth, temperature, RDO, salinity, and $\mathrm{pH}$ were measured in four locations. Across the four sites, depth ranged from 1.5 to $5.2 \mathrm{~m}$, illustrating minimal depth variability compared to sediment locations. Green Bay Cave, Manmade Cave, and Shark's Hole were shallow, ranging around 1.5 to 3 meters depth, while Flatt's Inlet was between 4 and 5.2 meters, depending on the proximity to the tidal channel (Figure 5A). Temperature, dissolved oxygen, and $\mathrm{pH}$ showed minimal to no variability across the four sampling locations, where temperature ranged from 28 to $28.9^{\circ} \mathrm{C}$ (Figure 5B), rugged dissolved oxygen ranged from 5.75 to $7.4 \mathrm{mg} / \mathrm{L}$ (Figure 5D), and pH ranged from 7.69 to 7.71 (Figure 5E). However, salinity followed similar trajectories as salinity measurements in sediment sampling, ranging from 3.1 to $37.6 \mathrm{psu}$, showing changes from mesohaline, brackish conditions to euhaline, ocean conditions (Figure $5 \mathrm{C})$.

To obtain an expanded dataset, winter samples were taken on January $18^{\text {th }}, 2019$. Here, physical and geochemical parameters at five sites over one full tidal cycle (incoming, slack, outgoing) were recorded (Figure 6A-E). Unlike summer samples, depth distribution showed more variability, ranging from 1 to 11 meters, from the shallowest at Manmade Cave to the 
deepest at Post Office Cave in Hamilton's Parish (Figure 6A). Similar to the summer samples, Green Bay Cave, Manmade Cave, and Shark's Hole were all shallow and remained in the range of 1 to 4 meters depth (Figure 6A). Temperature, salinity, dissolved oxygen, and pH illustrated little to no variability, where temperature ranged from 18.4 to $19.1{ }^{\circ} \mathrm{C}$ (Figure $6 \mathrm{~B}$ ), salinity ranged from 36.4 to 37.1 psu (Figure 6C), rugged dissolved oxygen ranged from 6.95 to 7.85 mg/L (Figure 6D), and $\mathrm{pH}$ ranged from 8.14 to 8.22 (Figure 6E). Despite these negligible changes, it is important to note that temperature values were significantly lower than those of the summer samples $\left(18.4-19.1{ }^{\circ} \mathrm{C}\right.$ vs $\left.28-28.9^{\circ} \mathrm{C}\right)$, salinity was euhaline and less variable than the summer samples (36.4-37.1 psu vs 3.1-37.6 psu), dissolved oxygen was higher and less variable than the summer samples $(6.95-7.85 \mathrm{mg} / \mathrm{L}$ vs 5.75-7.4 $\mathrm{mg} / \mathrm{L})$, and $\mathrm{pH}$ values were significantly higher than the summer samples (8.14-8.22 vs 7.69-7.71).

Mass percentage distribution

Prior to count data results, mass distribution for each test/fragment-size interval were measured and recorded to understand the relationships between sampling location and its parameters and test/fragment-size abundance. In the coarse test/fragment-size interval, there was a moderately increasing trend in mass percentage from northeast along the transect from sample site 4 to site 5 , then followed a significant drop in percentage until site 1 , then a dramatic spike from site 1 to site 8 , and concluded with a drop from site 8 to site 2 (Figure 7A). In the medium test/fragment-size interval, there is no discernable pattern of distribution, where sites 3 and 4 were distinctly higher in abundance and sites 1,2 , and 10 were distinctly lower in abundance than any other sites (Figure 7B). In the fine test/fragment-size interval, mass percentage showed little variability throughout the sites except for a large peak at site 1 (Figure 7C). In the very fine test/fragment-size interval, there was an increasing trend from site 4 to site 10 , but followed a 
drop in abundance from site 10 towards the northeast until site 2, which exhibited an increase in abundance (Figure 7D). However, it is important to note that mass percentages in the very fine test/fragment-size interval only reached a maximum abundance of $28.76 \%$ in any sampling site (Figure 7D). Overall, there seemed to be several trends in mass abundance in test/fragment-size intervals; however, these relationships collectively do not appear to be definitive.

When analyzing across test/fragment-size fractions, sampling sites 1 and 2 exhibited an increase in mass abundance as test/fragment-size fractions decreased, while sites $3,4,5,7$, and 8 exhibited a decrease in mass abundance (Figure 7). Sampling site 6 stayed consistent across all four test/fragment-size fractions, while site 10 illustrated a bimodal pattern, where mass abundance decreased from coarse to medium but increased from fine to very fine test/fragmentsize fractions (Figure 7). Based on the distribution of the sampling sites (Figure 2), there is no discernable spatial correlation in either clustering or environmental parameters. Thus, the mass distribution in each test/fragment-size interval at each sampling location lacked a recognizable, definitive relationship between and across sampling sites and test/fragment-size intervals.

\section{Count data distribution}

Count results for Quinqueloculina \& Triloculina spp. showed a broad range of variability in the larger test-size intervals (coarse and medium), while the smaller test-size intervals showed increasingly less and less variability (fine and very fine) (Figure 8). In the coarse test-size interval, there was a moderately increasing trend in test counts from site 4 to site 10 , then a decreasing trend towards the northeast until site 8 , ranging from 36 to 81 . In the medium test-size interval, there was a similar trend in counts as coarse test-size interval, ranging from 35 to 66 tests. In the fine test-size interval, there was neither an increasing nor decreasing trend in counts from site 1 to site 10, ranging from 33 to 45 tests. In the very fine test-size interval, there was 
similar lack of a trend in counts as the fine test-size interval, ranging from 20 to 31 tests (Figure 8). Based on the plotted data, it appeared that as test-sizes decreased, there were two important observations: (1) variability decreased; and (2) overall number of tests decreased (58.5 average $\rightarrow 50.5$ average $\rightarrow 39$ average $\rightarrow 25.5$ average). Thus, the distinct pattern seen in Quinqueloculina \& Triloculina spp. test counts was highly dependent on test-size intervals, both in variability and overall test abundance.

Count results for Homotrema rubrum fragments follow a pattern with Quinqueloculina \& Triloculina spp. tests, where larger fragment-size intervals showed greater variability, while smaller fragment-size intervals exhibited less and less variability in fragment counts (Figure 9). In the coarse fragment-size interval, there were three changes in trend direction, where counts decreased from site 4 to site 10 , increased from site 10 to site 1 , then decreased from site 1 to site 1, ranging from 19 to 63 fragments. In the medium fragment-size interval, there was a similar, moderately trend in counts as coarse fragment-size interval, ranging from 19 to 57 fragments. The fine fragment-size interval also exhibited the similar directional changes in trend, but was lower in count magnitude, ranging from 24 to 49 fragments. In the very fine fragment-size interval, there was a slight decrease in counts, ranging from 26 to 40 fragments (Figure 9). From these data, it appears that as fragment-sizes decreased, fragment count variability decreased. However, the overall fragment counts did not significantly decrease from decreasing fragmentsize intervals (41 average $\rightarrow 38$ average $\rightarrow 36.5 \rightarrow 33$ ) when compared to Quinqueloculina \& Triloculina spp. test counts (58.5 average $\rightarrow 50.5$ average $\rightarrow 39$ average $\rightarrow 25.5$ average). Thus, the distinct pattern seen in Homotrema rubrum fragment counts was highly dependent on fragment-size intervals. There is a significant difference due to variability and insignificantly in overall test abundance. 
Due to an absence of tests in the coarse test-size interval, Elphidium spp. count analysis was performed in medium, fine, and very fine test-size intervals. In contrast to Quinqueloculina \& Triloculina spp. test and Homotrema rubrum fragment counts, Elphidium spp. test-count results showed less variability in the medium test-size interval, while smaller test-size intervals (fine and very fine) exhibited greater variability (Figure 10). In the medium test-size interval, there was neither an increasing nor decreasing trend in counts, ranging from 4 to 11 tests. In the fine test-size interval, there was no trend in counts, similar to the coarse test-size interval, ranging from 3 to 14 tests. In the very fine test-size interval, there was an increasing trend in counts from site 4 to site 5 and from site 1 to site 2 , and a decreasing trend from site 5 to site 1 , ranging from 5 to 18 tests. From these data, it appears that as test-size interval decreased, variability and overall test counts increased (7.5 average $\rightarrow 8.5$ average $\rightarrow 11.5$ average). Thus, the pattern seen in Elphidium spp. test counts was moderately dependent on test-size intervals, both in variability and overall test counts.

Like Elphidium spp., no tests of Ammonia beccarii in the coarse test-size fraction were found and, therefore, count analyses were only performed in medium, fine, and very fine testsize intervals. Additionally, similar to Elphidium spp., Ammonia becarii test-count results showed less variability in the medium test-size interval, while greater variability in the smaller test-size intervals (fine and very fine) (Figure 11). In the medium test-size interval, there was neither an increasing nor decreasing trend in counts, ranging from 3 to 12 tests. In the fine testsize interval, there was also no trend in counts but ranged from 13 to 24 tests. In the very fine test-size interval, there was a slightly increasing trend in counts from site 5 to site 1 , ranging from 24 to 33 tests. From these plots, it appears that as test-size interval decreased, variability increased and overall test counts significantly increased ( 7.5 average $\rightarrow 18.5$ average $\rightarrow 28.5$ 
average). Thus, the pattern seen in Elphidium spp. test counts was highly dependent on test-size intervals, both in variability and overall test counts.

Normality testing

There were 14 Foraminifera test/fragment count intervals assessed for normality/nonnormality using the Shapiro-Wilk Normality Test. If at least $3 / 4^{\text {th }}$ of the taxa data fails to reject $\mathrm{H}_{\mathrm{o}}$ (not enough evidence to conclude that data is non-normal), parametric statistical methods were used. In this case, at least 11 taxa datasets need to fail to reject $\mathrm{H}_{\mathrm{o}}$ to proceed with parametric assumptions. Otherwise, non-parametric statistical methods were implemented with parametric methods laterally visualized for comparison.

In the Quinqueloculina \& Triloculina spp. count data, the medium test-size interval was the only one that rejected the null hypothesis, while the other three failed to see any nonnormality in the data (Table 1). On a similar note, in Homotrema rubrum count data, the very fine test-size interval was the only one rejecting $\mathrm{H}_{\mathrm{o}}$ while the other three intervals suggested a normal distribution. However, categories in both Elphidium spp. and Ammonia beccarii had a majority of hypothetical rejections, where four of the six normality tests rejected $\mathrm{H}_{\mathrm{o}}$ (very fine test-size fraction for Elphidium spp. and fine test-size fraction for Ammonia beccarii) (Table 1). It can also be noted that any Shapiro-Wilk test resulting in a W-statistic greater than 0.9 will result in an insignificant p-value greater than 0.05 (Table 1). Thus, because only 8 failed to reject $\mathrm{H}_{\mathrm{o}}$, which was not $3 / 4^{\text {th }}$ of the count data, profile and geospatial analyses were conducted under non-parametric assumptions and methodology. 


\section{Profile Analysis \\ $\underline{\text { Predictive Models }}$}

\section{Quinqueloculina \& Triloculina spp.}

Regression analysis is commonly known as one of the most helpful and insightful techniques in quantifying relationships, variable influences, and futuristic outputs. In this study, quantile regression was used as the primary tool; however, visualization was plotted alongside Poisson and linear regression models for comparison. All four test-size intervals of Quinqueloculina \& Triloculina spp. were plotted against depth (for visual), and modeled against a combination of depth, temperature, salinity, rugged dissolved oxygen, and $\mathrm{pH}$ (Figure 12). In the coarse test-size fraction Quinqueloculina \& Triloculina spp. plot, all three regression models (quantile, Poisson, linear) followed the same trajectory (Figure 12A). Stepwise optimization yielded the same inclusion-exclusion of variables, including depth, temperature, and salinity as significant variables to test count, while finding rugged dissolved oxygen and $\mathrm{pH}$ as insignificant variables related to test count (Table 2). Additionally, it can be observed that the modeling formulas included interaction terms (Depth:Temperature), which indicated that temperature's significance to test count variability depended on the variability of depth as an explanatory factor (Table 2). Thus, because the stepwise-regression process yielded similar, optimal models (with variable interaction) across parametric and non-parametric techniques, the visualization followed the same trend.

In the medium test-size fraction plot of Quinqueloculina \& Triloculina spp., quantile regression noticeably deviated from the other models, specifically around depths of 7 to 10 meters (Figure 12B). Here, the stepwise optimization varied across the three models, where quantile regression identified depth, temperature, and RDO as significant to test count, and excluded salinity and $\mathrm{pH}$ as insignificant factors. Meanwhile, Poisson and linear regressions both 
included depth, temperature, $\mathrm{RDO}$, and $\mathrm{pH}$, and only excluded salinity (Table 2). It is also important to note that because the quantile regression model excluded $\mathrm{pH}$, the model formula varied from the formulas of the other two techniques, where coefficients significantly varied in their direction and magnitude of influence (Table 2). Unlike coarse test-size fraction of Quinqueloculina \& Triloculina spp., quantile and linear models in this test-size fraction did not include the interaction between depth and temperature but, instead, included the interaction between depth and RDO as significant to test count results (Table 2). Here, quantile and linear regression showed that dissolved oxygen's significance is related to test-count variability dependent on depth. Thus, when the stepwise-regression process produced different inclusionexclusion combinations, visualization was different, and those differences illustrated changes in coefficient direction and magnitude towards test-count results.

In the plot of the fine test-size fraction of Quinqueloculina \& Triloculina spp., quantile and linear curves were indistinguishable, while the Poisson curve exhibited a positive, linear trend, deviating from the other two models at around 6 to 11 meters depth and 14 to 16 meters depth (Figure 12C). Stepwise optimization exhibited limited inclusion, where quantile and linear regressions both included depth, $\mathrm{RDO}$, and $\mathrm{pH}$ as significant related to test count, and identified temperature and salinity as insignificant to test count. In contrast, Poisson regression only identified depth as a significant factor and found the others as insignificant (Table 2). The model formulas and coefficients in quantile and linear regressions were almost identical in direction and magnitude, which continued to illustrate the relationship between inclusion-exclusion and visualized trajectory (Table 2). Unlike the previous two test-size intervals, there were no interaction terms included in this test-size fraction's stepwise optimization, suggesting no inter- 
variable correlation and dependence. Furthermore, this decrease in correlation appeared to decrease the number of variables included in the stepwise optimization process (Table 2).

Similar to the fine test-size fraction Quinqueloculina \& Triloculina spp. plot, quantile and linear models were almost indistinguishable in trajectory while the Poisson model followed a linear trend in the very fine test-size fraction plot of Quinqueloculina \& Triloculina spp. (Figure 12D). However, quantile and linear models deviated from one another around 12 to 15 meters depth, and Poisson and linear regressions were indistinguishable (Figure 12D). Here, stepwise optimization resulted in different combinations across all three models, where quantile regression included everything but salinity as being significant factors relative to test count, Poisson regression excluded all variables as significant to test count, and linear regression included depth, temperature, and RDO as significant. Poisson regression excluded salinity and $\mathrm{pH}$ as significant factors related to test count (Table 2). In terms of interaction, both quantile and linear models included the interaction between depth and temperature, exhibiting a similar combination of environmental parameters and interaction terms comparable to the coarse test-size interval (Table 2). Despite these interactive similarities, the inclusion of $\mathrm{pH}$ in the quantile model and exclusion of $\mathrm{pH}$ in the linear model continued to illustrate the correlation between variable inclusion-exclusion and visual differences.

Overall, there did not seem to be a discernable relationship between test-size interval and the inclusion-exclusion of environmental parameters. It is important to note that salinity did not seem to be a significant variable in any test-size interval except in the coarse test-size fraction (Table 2). When comparing parametric and non-parametric techniques against one another, there was a strong correlation between inclusion-exclusion and visual deviation. That is, the regression curves deviated from one another. Nevertheless, the quantile models and stepwise optimization 
for all test-size intervals of Quinqueloculina \& Triloculina spp. yielded inconsistent results in variable selection and combinations of environmental parameters.

\section{Homotrema rubrum}

Analyzing and modeling Homotrema rubrum was limited because fragments were counted instead of full tests. However, Figure 13 plots fragment counts against depth for visualization and are modeled against environmental parameters (depth, temperature, salinity, $\mathrm{RDO}, \mathrm{pH})$. In the coarse fragment-size fraction plot of Homotrema rubrum plot, quantile, Poisson, and linear curves were indistinguishable, both in trajectory and variability (Figure 13A). Here, stepwise optimization yielded identical inclusion-exclusion combinations of environmental parameters, where all three regressions included depth, temperature, salinity, and RDO, and excluded $\mathrm{pH}$ as significant factors related to fragment-count variability (Table 3 ). Interestingly, it can be observed that quantile and Poisson models excluded interaction terms from their optimization process, while the linear model included interactions between depth and temperature, and depth and RDO (Table 3). From a non-parametric standpoint, interaction terms did not significantly contribute to fragment-count variability, while parametric techniques including linear regression found them to be significant. Thus, despite the disparities between parametric and non-parametric assumptions, when inclusion-exclusion combinations of environmental factors were similar across each modeling technique, the visual trajectory of all three models was indistinguishable for $H$. rubrum fragments at the coarse fraction.

In the medium fragment-size fraction Homotrema rubrum plot, quantile regression completely deviated from Poisson and linear regression, both of which were visually indistinguishable (Figure 13B). All three models exhibited a similar shape, but the quantile curve departed from the common trajectory throughout the plot. The quantile curve intersects the other 
two curves at depths of 7 and 17 meters (Figure 13B). Here, stepwise optimization included all five environmental parameters across all three models as significant variables related to fragment-count variability (Table 3). However, the visual deviations seen in the plot could be explained by the inclusion-exclusion of interaction terms, where quantile regression excluded interaction terms, while Poisson and linear regressions included both interactions between depth and temperature, and depth and RDO (Table 3). Similar to the coarse fragment-size interval, the non-parametric technique did not identify interaction terms to significantly contribute to fragment-count variability, while parametric techniques found them as significant. Thus, despite all three models including the same individual, environmental parameters, the inclusionexclusion of interaction terms appears to be correlated with visual deviations in the plot.

Similar to the coarse fragment-size fraction H. rubrum plot, all three modeling techniques in the fine fragment-size fraction plot produced similar-to-indistinguishable curves, although the Poisson model slightly deviated from quantile and linear models at depths of 15 to 17 meters (Figure 13C). In stepwise optimization, quantile and linear models included all five parameters (depth, temperature, salinity, $\mathrm{RDO}, \mathrm{pH}$ ) as significant related to fragment counts, and the Poisson model included depth, $\mathrm{RDO}$, and $\mathrm{pH}$, and excluded temperature and salinity (Table 3). Linear regression was the only model that included the interaction between depth and temperature. However, it did not seem to offset its trajectory from the quantile model, which did not consider the interaction term as significant related to fragment-count variability (Table 3). Identical to the previous fragment-size fractions, the non-parametric technique in the fine fragment-size fraction did not identify inter-variable interaction as a significant factor related to fragment counts, but one of the parametric techniques (linear regression) did find them as significant. Overall, despite the lack of visual deviation among the three models' curvatures in the fine fragment-size 
fraction, their model formulas and inclusion-exclusion combinations (including interaction terms) were quite different. These results contradict the relationship between inclusion-exclusion and visual trajectories, seen in the previous fragment-size intervals of Homotema rubrum and Quinqueloculina \& Triloculina spp.

Similar to the coarse and fine fragment-size fractions of $H$. rubrum, the very fine fragment-size fraction plot illustrated similar-to-indistinguishable curves across all three models (quantile, Poisson, linear) (Figure 13D). Linear regression seemed to slightly deviate from the other two in the shallower depths from 2 to 5 meters, and Poisson slightly deviated from quantile and linear from the depths of 12 to 14 meters (Figure 13D). The stepwise optimization yielded different inclusion-exclusion combinations when compared to the plot in Figure 13D, where quantile and Poisson models included depth, temperature, and RDO as significant, and excluded salinity and $\mathrm{pH}$ as insignificant. However, the linear model found all five parameters to be significant (Table 3). In terms of interaction, all three regression methods found the interaction between depth and temperature as a significant term related to fragment counts, but linear regression additionally found the interaction between depth and RDO to influence fragmentcount variability (Table 3). Despite differences in inclusion-exclusion of both individual parameters and interaction terms, there is no visual deviation across these three models; this trend is similar to the fine fragment-size interval. Furthermore, this contradicts the relationship between inclusion-exclusion and visual trajectories as identified in the coarse and medium sizefractions of $H$. rubrum as well as Quinqueloculina \& Triloculina spp. test-size fractions.

Overall, there were two patterns identified as noteworthy. First, only the very fine fragment-size fraction of Homotrema rubrum found interaction terms to be significant contributors (quantile models). Second, compared to the test-size fractions of Quinqueloculina \& 
Triloculina spp., these modeling techniques consistently included more parameters and interaction terms in the fragment-size fractions of $H$. rubrum. Despite these patterns, there was still an absence of discernable relationships between inclusion-exclusion combinations and fragment-size intervals. Additionally, unlike test-size fractions of Quinqueloculina \& Triloculina spp., there was not a strong correlation between inclusion-exclusion and visual deviation. In other words, the regression curves did not significantly deviate from one another based on different parameter combinations. Nevertheless, the quantile models and stepwise optimization for all fragment-size intervals of Homotrema rubrum yielded inconsistent results in variable selection and its relationships to fragment-size intervals and visual trajectory.

\section{Elphidium spp.}

Only medium, fine, and very fine test-size fractions were assessed and modeled for Elphidium spp.. As a result, comparative analyses relating test-size fractions of Quinqueloculina \& Triloculina spp. and fragment-size fractions of Homotrema rubrum only involved these three categories. In the medium test-size fraction Elphidium spp. plot, all three models were distinguishable from one another, where quantile and Poisson models followed the similar shape and trajectory, although unaligned, and the linear model followed a completely different trend (Figure 14A). An additional observation was the visual accuracy of the models themselves. The curves began to deviate from the scattered data around 12 meters depth, which was not seen in the plots of test/fragment-size intervals of Quinqueloculina \& Triloculina spp. and Homotrema rubrum (Figure 14A). Stepwise optimization yielded predominantly exclusive results when compared to previous models, where quantile and Poisson regressions only included depth as a significant factor, while finding the remaining parameters as insignificant related to test count. In contrast, linear regression included depth, temperature, and salinity as significant factors, and 
identified $\mathrm{RDO}$ and $\mathrm{pH}$ as insignificant factors (Table 4). Unlike previous taxa, the medium testsize fraction of Elphidium spp. included a squared term (Depth $\left.{ }^{2}\right)$ as a significant contributor to test-count variability in addition to the individual parameter of depth (Table 4). In other words, because depth had a positive coefficient and depth ${ }^{2}$ had a negative coefficient across all three models, the effect of depth on test count variability decreased as depth increased in magnitude. Thus, overall, there were two patterns in this test-size fraction: (1) because quantile and Poisson regressions only included depth and depth ${ }^{2}$ as significant parameters, their visual trajectories were very similar; and (2) because inclusion-exclusion combinations were predominantly exclusive, deviation from scattered data was prevalent.

In the fine test-size fraction Elphidium spp. plot, all three model trends were similar or indistinguishable in trajectory and shape, and several deviations from the scattered data are identified. This is similar to that seen in the medium test-size fraction Elphidium spp. plot (Figure 14B). At depths of 7, 12, and 15 meters, the models were visually inaccurate and significantly deviated from the test-count values (Figure 14B). Stepwise optimization yielded similar model pairing between quantile and Poisson regressions, which both included depth, temperature, salinity, and $\mathrm{pH}$ as significant contributors, while only identifying RDO as an insignificant factor. In contrast, linear regression included all five parameters (Table 4). Here, all three models included the interaction between depth and temperature, finding that temperature's effect on test-count variability was dependent on the variability of depth. Additionally, unlike the medium test-size fraction of Elphidium spp., depth ${ }^{2}$ was not included in all three models. This indicates that depth had a linear effect on test count, rather than a quadratic effect throughout this test-size fraction (Table 4). In summary, because stepwise regression excluded $\mathrm{pH}$ from quantile 
and Poisson models and retained it in the linear model, results for fine test-size fraction Elphidium spp. showed no relationship between inclusion-exclusion and visual trajectory.

All three models were indistinguishable in shape in the very fine test-size fraction Elphidium spp. plot, but quantile regression significantly deviated from Poisson and linear regression at depth ranges of 4 to 7 meters, and 10 to 17 meters (Figure 14C). Similar to the fine test-size fraction, models in the very fine test-size fraction deviated from the scattered data at depths of 7 and 12 meters, indicating visual deviations at these depth ranges (Figure 14C). Stepwise optimization yielded predominantly exclusive combinations of parameters, where the quantile model included depth, temperature, salinity, and pH, and excluded RDO; meanwhile, Poisson and linear models included all five parameters (Table 4). Additionally, the interaction between depth and temperature was included across all three models, indicating a relationship between temperature's influence on test-count variability and depth's explanatory variability (Table 4). Similar to the fine test-size fraction, all three models in the very fine test-size fraction did not include depth ${ }^{2}$ as a significant squared term, signifying depth's linear effect on test count (Table 4). In an inconsistent manner, the plot and stepwise optimization of very fine test-size fraction of Elphidium spp. data reversed the relationship between variable inclusion and visual trajectory. This is because the similar inclusion-exclusion combinations across all three models resulted in visual deviation.

Overall, there were three patterns noteworthy. First, as the test-size fraction of Elphidium spp. decreased, parameter inclusion increased across all three models. Second, at the medium test-size fraction, depth appeared to have a quadratic effect on test-count variability and there was no inter-variable interaction. Third, temperature's effect on test-count variability was dependent on depth in both fine and very fine test-size intervals. Additionally, similar to size- 
fractions of Homotrema rubrum, there was not a strong correlation between inclusion-exclusion and visual deviation. That is, the regression curves did not significantly deviate from one another based on differing combinations of parameters. Unlike test-size fractions of Quinqueloculina \& Triloculina spp. and fragment-size fractions of Homotrema rubrum, the quantile models and stepwise optimization for all test-size intervals of Elphidium spp. yielded consistent results in variable selection and its relationship to test-size interval, but inconsistent results in visual trajectory.

\section{$\underline{\text { Ammonia beccarii }}$}

Models of Ammonia beccarii involved medium, fine, very fine test-size fractions. This constrained the analyses to other taxa with the same test/fragment-size intervals. In the medium test-size fraction Ammonia beccarii plot, all three models were similar in trajectory but distinguishable in visual deviation and variability (Figure 15A). From depths of 12 to 17 meters, the model curves significantly deviated from the scattered data, illustrating model inaccuracy at these deeper depths. Stepwise optimization yielded different inclusion-exclusion combinations across all three models, whereas quantile regression only included depth as a significant factor related to test-count variability. Stepwise optimization identified the remaining variables as insignificant. Poisson regression included depth, temperature, and $\mathrm{pH}$ as significant, and identified temperature as insignificant; linear regression included all five parameters (Table 5). Poisson and linear models both included the interaction between depth and temperature, while the quantile model did not include any interactive terms (Table 5). Here, quantile algorithms did not find temperature's effect on test-count variability dependent on depth. However, unlike the Poisson and linear models, the quantile model in the medium test-size fraction of Elphidium spp. included depth ${ }^{2}$ as a significant contributor to test count. In other words, because depth had a 
positive coefficient and depth ${ }^{2}$ had a negative coefficient, the effect of depth on test count decreased as depth increased in magnitude (Table 5). Thus, the visual deviation seen in Figure $15 \mathrm{~A}$ corresponds with the drastic differences in variable inclusion across all three models, which involved inclusion-exclusion of individual parameters, interaction terms, and squared terms.

In the fine test-size fraction plot of Ammonia beccarii, quantile and Poisson models were indistinguishable in trajectory and shape, while the linear model deviated from these two at depth ranges of 0 to 12 meters and 15 to 18 meters. An additional, noteworthy observation was that quantile and Poisson curves were both distinctly linear, while the linear curve appeared to be more quadratic in shape (Figure 15B). Stepwise optimization paired quantile and Poisson models with similar inclusion-exclusion combinations, where both regressions included depth as the only significant contributor to test-count variability and excluded the rest of the parameters (Table 5). Meanwhile, linear regression included all five parameters as significant when related to test count (Table 5). In terms of interaction, neither quantile nor Poisson models included interaction terms (i.e., depth and temperature). However, linear regression found temperature's influence on test-count variability dependent on depth (Table 5). Unlike the medium test-size fraction, the quantile model in the fine test-size fraction did not include depth ${ }^{2}$, but the linear model did. In this case, quantile regression found depth to have a linear effect on test count, rather than a quadratic effect. Thus, overall, the visual deviation between all three models (Figure 15B) corresponds with the quantile and Poisson models' similar inclusion-exclusion combinations, which drastically differ from the linear model.

Similar to the fine test-size fraction Ammonia beccarii plot, the plot of the very fine testsize fraction Ammonia beccarii exhibited similar, visual deviations, whereas quantile and Poisson curves were indistinguishable, and the linear curve deviated from the other two models 
at depth ranges of 0 to 10 meters and 16 to 17 meters (Figure 15C). Additionally, quantile and Poisson models display a linear trend, while the linear model illustrated a quadratic shape, which was similar to the previous test-size fraction (Figure 15C). Stepwise optimization paired quantile and Poisson regressions with similar inclusion-exclusion, only including depth as significant to test-count variability and excluding the rest of the parameters. Linear regression included all five parameters as significant contributors to test count (Table 5). Identical to the combinations listed in the fine test-size fraction, quantile and Poisson regressions did not include any interaction or squared terms. In contrast, linear regression included the interaction between depth and temperature (Table 5). Thus, from these results, there were two observations to be made. First, depth had a linear effect on test count, instead of a quadratic effect. Second, the visual deviations in Figure 15C correspond with quantile and Poisson models' similarities and their differences from the linear model.

In summary, two noteworthy patterns emerge from these data. First, there was no discernable relationship between test-size interval and variable inclusion. Second, the results of the quantile model were almost identical across all three test-size intervals, except the medium test-size fraction's inclusion of depth ${ }^{2}$. Additionally, similar to test-size fractions of Quinqueloculina \& Triloculina spp., there was a correlation between inclusion-exclusion and visual deviation. That is, the regression curves significantly deviated from one another based on differing parameter combinations. Similar to test-size fractions of Elphidium spp., all test-size fractions of Ammonia beccarii yielded consistent results in variable selection and their relationship to test-size intervals and, in this case, consistent results in visual trajectory, as well. 


\section{$\underline{\text { Multivariate Models }}$}

\section{Coarse test/fragment-size interval}

\section{Global Hypothesis}

The first step in the non-parametric Multivariate Analysis of Variance (npMANOVA) was to fulfill the Global Hypothesis, under the following assumptions: $\mathrm{H}_{\mathrm{o}}$ : All factor levels have the same effect on dead test and fragment counts; $\mathrm{H}_{\mathrm{a}}$ : At least one factor level has a different effect on dead test and fragment counts. The main differences between predictive modeling and multivariate modeling are the variables in focus. In predictive models, one response variable is investigated against multiple explanatory variables, while in multivariate models, multiple response variables are investigated against one explanatory variable. In this case, all four taxa at each test/fragment-size interval were modeled against one of the five environmental parameters.

At the coarse test/fragment-size interval, five Global Hypothesis ANOVA tests were performed on the five parameters (depth, temperature, salinity, $\mathrm{RDO}$, and $\mathrm{pH}$ ) using the ANOVA-Type Test, LH Test, BNP Test, and Wilks Lambda protocols. Because rank order values are singular and data in both coarse test-size fraction of Elphidium spp. and Ammonia beccarii is lacking, the only test that worked with singular-ranked data was the ANOVA-Type Test. The ANOVA-Type Test for depth, temperature, salinity, rugged dissolved oxygen, and $\mathrm{pH}$ were all statistically significant at less than a 0.05 level of significance (Table 6). The p-values and p-values derived from 1000 permutation tests for depth, salinity, RDO, and pH were highly significant (Table 6). The exception is temperature, where a highly significant p-value (0.034) was found, although it was insignificant under permutation (0.068), suggesting that any interpretation should be with caution (Table 6B). An important observation to note is the teststatistic values, which showed that test-statistics above the value of 6 would result in higher 
significance (Table 6). Thus, due to the statistical significance of the Global Hypotheses for all five parameters, the Subset Algorithm for test/fragment counts was implemented.

II. Subset Algorithm on coarse test/fragment counts

Following the Global Hypothesis, Subset Algorithms tested the following hypotheses: $\mathrm{H}_{\mathrm{o}}$ : Counts for all taxa are equal; $\mathrm{H}_{\mathrm{a}}$ : At least one taxon is not equal. In other words, this algorithm was used to differentiate taxa, if possible. According to Table 7, Homotrema rubrum was considered a $1^{\text {st }}$ order, statistically distinguishable taxon, and Quinqueloculina \& Triloculina spp. were considered a $2^{\text {nd }}$ order statistically distinguishable taxa, while Elphidium spp. and Ammonia beccarii were both statistically indistinguishable from one another at the coarse test/fragmentsize interval. The two orders of statistically distinguishable taxa are categorized based on the magnitude of statistical difference from one another. Hence, Homotrema rubrum was the first taxon determined by the algorithm as statistically different from the other three taxa, whereas Quinqueloculina \& Triloculina spp. was the second taxonomic category determined as different. Due to an absence of data, Elphidium spp. and Ammonia beccarii were indistinguishable from one another (Table 7). This pattern was identical across depth, salinity, RDO, and pH. In contrast, Subset Algorithms for temperature were unable to differentiate which taxa were statistically distinguishable (Table 7).

\section{Subset Algorithm on environmental parameters}

The second set of Subset Algorithms tested the effects of the factor levels within each environmental parameter, under the following assumptions: $\mathrm{H}_{\mathrm{o}}$ : All factor levels in environmental parameter contributed equally to significant differences in counts. $\mathrm{H}_{\mathrm{a}}$ : At least one factor level in the environmental parameters contributed to significant differences in counts. At the coarse test/fragment-size interval, the significant differences in taxa were modeled against 
three depth zones: (1) Shallow Sandy Zone (0-10 meters); (2) Oculina Zone (10-18 meters); (3) Subthermocline Zone (18-25 meters). The Subset Algorithm for depth found the Shallow Sandy Zone as the $1^{\text {st }}$ order, statistically distinguishable zone; the Subthermocline Zone as the $2^{\text {nd }}$ order, statistically distinguishable zone; and, by process of elimination, the Oculina Zone as the $3^{\text {rd }}$ order, statistically distinguishable zone (Table 8). Here, the Shallow Sandy Zone was the first depth factor-level to be identified as contributing differently to significant differences in test/fragment counts. The Subthermocline Zone was the second factor-level to be identified to contribute differently to significant differences in test/fragment counts and, by default, the Oculina Zone was the last to be ordered (Table 8).

When modeling the coarse test/fragment-size interval against temperature, the significant differences in taxa were tested against three temperature zones: (1) Mixed Layer $\left(26-28{ }^{\circ} \mathrm{C}\right)$; (2) Thermocline Layer $\left(24-26{ }^{\circ} \mathrm{C}\right)$; and (3) Subthermocline Layer $\left(22-24{ }^{\circ} \mathrm{C}\right)$ (Table 9). The Subset Algorithm for temperature found the Mixed Layer as the $1^{\text {st }}$ order, statistically distinguishable zone, but was unable to differentiate the Thermocline Layer from the Subthermocline Layer (Table 9). Here, the Mixed Layer was the first temperature factor-level to be identified as contributing to significant differences in test/fragment counts.

When modeling the coarse test/fragment-size interval against salinity, the significant differences in taxa were tested against three salinity zones: (1) Mesohaline (2-10 psu); (2) Polyhaline (10-35 psu); and (3) Euhaline (35+ psu) (Table 10). The Subset Algorithm for salinity found the Mesohaline zone as the $1^{\text {st }}$ order, statistically distinguishable zone, but was unable to differentiate the Polyhaline zone from the Euhaline zone (Table 10). Here, the Mesohaline zone was the first salinity factor-level to be identified as contributing to significant differences in test/fragment counts. 
When modeling the coarse test/fragment-size interval against RDO, the significant differences in taxa were tested against four RDO zones: (1) 0-2 mg/L; (2) 2-4 mg/L; (3) 4-6 mg/L; and (4) 6-9 mg/L (Table 11). The Subset Algorithm for RDO found the 4-6 mg/L and 6-9 $\mathrm{mg} / \mathrm{L}$ zones as $2^{\text {nd }}$ order, statistically distinguishable zones, but $0-2 \mathrm{mg} / \mathrm{L}$ and $2-4 \mathrm{mg} / \mathrm{L}$ zones are statistically indistinguishable zones (Table 11). Here, the 4-6 mg/L and 6-9 mg/L zones were the first RDO factor-levels to be identified as contributing to significant differences in test/fragment counts. However, the algorithms were unable to differentiate 4-6 mg/L and 6-9 mg/L from one another, despite being able to differentiate significance between the two zones.

When modeling the coarse test/fragment-size interval against $\mathrm{pH}$, the significant differences in taxa were tested against three pH zones: (1) 8.0-8.2; (2) 7.8-8.0; and (3) 7.6-7.8 (Table 12). The Subset Algorithm for $\mathrm{pH}$ found the $7.6-7.8$ zone as the $1^{\text {st }}$ order, statistically distinguishable zone, but was unable to differentiate the 8.0-8.2 and 7.8-8.0 zones (Table 12). Here, the 7.6-7.8 zone was the first $\mathrm{pH}$ factor-level to be identified as contributing to significant differences in test/fragment counts.

\section{Relative Probabilities}

Using the relative effects probability algorithms, the likelihood of test/fragment count abundance under a given factor-level for an environmental parameter were calculated for each taxon. These tests were done for each different test/fragment-size interval. Under the coarse test/fragment-size fraction, tested against depth zones, relative effect probabilities were calculated. The likelihood of finding the most Quinqueloculina \& Triloculina spp. tests in the Subthermocline Zone more than any other depth zone is 0.944 (Table 13A). The likelihood of finding the most Homotrema rubrum fragments in the Shallow Sandy Zone more than any other depth zone is 0.759 (Table 13A). The likelihood of finding the most Elphidium spp. tests in the 
Shallow Sandy Zone more than any other depth zone is 0.722 (Table 13A). The likelihood of finding the most Ammonia beccarii tests in any depth zone is 0.500 (Table 13A).

Under the coarse test/fragment-size fraction, tested against temperature zones, relative effect probabilities were calculated. The likelihood of finding the most Quinqueloculina \& Triloculina spp. tests in the Subthermocline Layer more than any other temperature zone is 0.944 (Table 13B). The likelihood of finding the most Homotrema rubrum fragments in the Mixed Layer more than any other temperature zone is 0.574 (Table 13B). The likelihood of finding the most Elphidium spp. tests in the Mixed Layer more than any temperature zone is 0.556 (Table 13B). The likelihood of finding the most Ammonia beccarii tests in any temperature zone is 0.500 (Table 13B).

Under the coarse test/fragment-size fraction, tested against salinity zones, relative effect probabilities were calculated. The likelihood of finding the most Quinqueloculina \& Triloculina spp. tests in the Euhaline Zone more than any other salinity zone is 0.944 (Table 13C). The likelihood of finding the most Homotrema rubrum fragments in the Mesohaline Zone more than any other salinity zone is 0.656 (Table 13C). The likelihood of finding the most Elphidium spp. tests in the Mesohaline Zone more than any salinity zone is 0.589 (Table 13C). The likelihood of finding the most Ammonia beccarii tests in any salinity zone is 0.500 (Table 13C).

Under the coarse test/fragment-size fraction, tested against RDO zones, relative effect probabilities were calculated. The likelihood of finding the most Quinqueloculina \& Triloculina spp. tests in the 0-2 mg/L zone more than any other RDO zone is 0.944 (Table 13D). The likelihood of finding the most Quinqueloculina \& Triloculina spp. tests in the $2-4 \mathrm{mg} / \mathrm{L}$ zone more than any other RDO zone is 0.833 (Table 13D). The likelihood of finding the most Homotrema rubrum fragments in the 6-9 $\mathrm{mg} / \mathrm{L}$ zone more than any other RDO zone is 0.694 
(Table 13D). The likelihood of finding the most Elphidium spp. tests in the 6-9 mg/L zone more than any RDO zone is 0.639 (Table 13D). The likelihood of finding the most Ammonia beccarii tests in any RDO zone is 0.500 (Table 13D).

Under the coarse test/fragment-size fraction, tested against $\mathrm{pH}$ zones, relative effect probabilities were calculated. The likelihood of finding the most Quinqueloculina \& Triloculina spp. tests in the 7.6-7.8 zone more than any other $\mathrm{pH}$ zone is 0.944 ; the likelihood of finding the most Quinqueloculina \& Triloculina spp. tests in the 7.8-8.0 zone more than any other $\mathrm{pH}$ zone is 0.546 (Table 13E). The likelihood of finding the most Homotrema rubrum fragments in the 8.0-8.2 zone more than any other $\mathrm{pH}$ zone is 0.617 (Table 13E). The likelihood of finding the most Elphidium spp. tests in the 8.0-8.2 zone more than any $\mathrm{pH}$ zone is 0.589 (Table $13 \mathrm{E}$ ). The likelihood of finding the most Ammonia beccarii tests in any $\mathrm{pH}$ zone is 0.500 (Table 13E).

\section{Medium test/fragment-size interval}

I. Global Hypothesis

At the medium test/fragment-size interval, five Global Hypothesis ANOVA tests were performed on the five parameters (depth, temperature, salinity, RDO, and $\mathrm{pH}$ ) using the ANOVA-Type Test, LH Test, BNP Test, and Wilks Lambda. The ANOVA-Type Test for depth, temperature, salinity, rugged dissolved oxygen, and $\mathrm{pH}$ were all statistically significant $(\mathrm{p}<0.05)$ (Table 14). The LH Test for depth, temperature, salinity, rugged dissolved oxygen, and $\mathrm{pH}$ were all statistically significant (Table 14). The BNP Test was only statistically significant for depth, salinity, and rugged dissolved oxygen (Table 14). The Wilks Lambda was statistically significant for depth, salinity, rugged dissolved oxygen, and $\mathrm{pH}$ (Table 14). An important observation to note was the test-statistic value of each test, which showed that ANOVA test-statistics above the value of 6 would result in higher significance, LT test-statistics above the value of 2.00 would 
result in higher significance, BNP test-statistics above 2.20 would result in higher significance, and Wilks Lambda test-statistics above 2.20 would result in higher significance (Table 14). Thus, due to the statistical significance of the Global Hypotheses for all five parameters, using at least one significant ANOVA test, the Subset Algorithm for test/fragment counts proceeded.

II. Subset Algorithm on medium test/fragment counts

Following the Global Hypothesis, Subset Algorithms testing the following hypotheses: $\mathrm{H}_{\mathrm{o}}$ : Counts for all taxa are equal; $\mathrm{H}_{\mathrm{a}}$ : At least one taxonomic group is not equal. In other words, this algorithm was used to differentiate one taxon from one another, if possible. According to Table 15, at the medium fragment-size interval, Homotrema rubrum was considered a $1^{\text {st }}$ order, statistically distinguishable taxon for depth and salinity, and $2^{\text {nd }}$ order statistically distinguishable taxon for temperature, RDO, and $\mathrm{pH}$. At the medium test-size interval, Quinqueloculina \& Triloculina spp. was considered a $2^{\text {nd }}$ order statistically distinguishable taxonomic group for all five parameters. At the medium test-size interval, Elphidium spp. was considered a $1^{\text {st }}$ order, statistically distinguishable taxon for temperature, statistically similar to Ammonia beccarii for depth, salinity, and RDO, and statistically indistinguishable for $\mathrm{pH}$. At the medium test-size interval, Ammonia beccarii was statistically similar to Elphidium spp. for all five parameters (Table 15). The two orders of statistically distinguishable taxa are categorized based on the magnitude of statistical difference from one another. For example, Homotrema rubrum was the first taxon determined by the algorithm as statistically different from the other three taxa for depth and salinity, Quinqueloculina \& Triloculina spp. was the second group determined as different for all five parameters, and Elphidium spp. and Ammonia beccarii were not statistically distinguishable from one another for depth, salinity, and RDO (Table 15). 


\section{Subset Algorithm on environmental parameters}

The second set of Subset Algorithms tested the effects of the levels within each environmental parameter, under the following assumptions: $\mathrm{H}_{\mathrm{o}}$ : All factor levels in environmental parameter contributed equally to significant differences in counts. $\mathrm{H}_{\mathrm{a}}$ : At least one factor level in environmental parameter contributed differently to significant differences in counts. At the medium test/fragment-size interval, the significant differences in taxa were modeled against three depth zones: (1) Shallow Sandy Zone (0-10 m); (2) Oculina Zone (10-18 m); and (3) Subthermocline Zone (18-25 m). The Subset Algorithm for depth found the Shallow Sandy Zone as the $1^{\text {st }}$ order, statistically distinguishable zone, and Oculina and Subthermocline zones as statistically similar (Table 16). Here, the Shallow Sandy Zone was the first depth factorlevel to be identified as contributing differently to significant differences in test/fragment counts, and the Oculina and Subthermocline zones were identified as influencing significant differences in test/fragment counts (Table 16).

When modeling the medium test/fragment-size interval against temperature, the significant differences in taxa were tested against three temperature zones: (1) Mixed Layer $\left(26-28{ }^{\circ} \mathrm{C}\right)$; (2) Thermocline Layer $\left(24-26{ }^{\circ} \mathrm{C}\right)$; and (3) Subthermocline Layer $\left(22-24{ }^{\circ} \mathrm{C}\right)$ (Table 17). The Subset Algorithm for temperature found the Subthermocline Layer as the $1^{\text {st }}$ order, statistically distinguishable zone, but was unable to differentiate the Mixed Layer from the Thermocline Layer (Table 17). Here, the Subthermocline Layer was the first temperature factor-level to be identified as contributing to significant differences in test/fragment counts.

When modeling the medium test/fragment-size interval against salinity, the significant differences in taxa were tested against three salinity zones: (1) Mesohaline (2-10 psu); (2) Polyhaline (10-35 psu); and (3) Euhaline (35+ psu) (Table 18). The Subset Algorithm for salinity 
found the Mesohaline zone as the $1^{\text {st }}$ order, statistically distinguishable zone, but the Polyhaline zone and the Euhaline zone are statistically indistinguishable (Table 18). Here, the Mesohaline zone was the first salinity factor-level to be identified as contributing to significant differences in test/fragment counts.

When modeling the medium test/fragment-size interval against RDO, the significant differences in taxa were tested against four RDO zones: (1) 0-2 mg/L; (2) 2-4 mg/L; (3) 4-6 mg/L; and (4) 6-9 mg/L (Table 19). The Subset Algorithm for RDO found the 4-6 mg/L zone as $1^{\text {st }}$ order, statistically distinguishable zone, $6-9 \mathrm{mg} / \mathrm{L}$ zone as the $2^{\text {nd }}$ order, statistically distinguishable zone, and 0-2 mg/L and 2-4 mg/L zones as statistically similar zones (Table 19). Hence, the 4-6 mg/L zone was the first RDO factor-levels to be identified as contributing to significant differences in test/fragment counts and the 6-9 $\mathrm{mg} / \mathrm{L}$ zone was the second RDO factor-levels to be identified as contributing to significant differences in test/fragment counts.

When modeling the medium test/fragment-size interval against $\mathrm{pH}$, the significant differences in taxa were tested against three pH zones: (1) 8.0-8.2; (2) 7.8-8.0; and (3) 7.6-7.8 (Table 20). The Subset Algorithm for $\mathrm{pH}$ found the 7.6-7.8 zone as the $1^{\text {st }}$ order, statistically distinguishable zone, the but was unable to differentiate the 8.0-8.2 and 7.8-8.0 zones (Table 20). Hence, the 7.6-7.8 zone was the first $\mathrm{pH}$ factor-level to be identified as contributing to significant differences in test/fragment counts.

\section{Relative Probabilities}

Using the relative effects probability algorithms, the likelihood of test/fragment count abundance under a given factor-level for an environmental parameter was calculated for each taxon, under different test/fragment-size intervals. Under the medium test/fragment-size fraction, tested against depth zones, relative effect probabilities were calculated. The likelihood of finding the most Quinqueloculina \& Triloculina spp. tests in the Subthermocline Zone more than any 
other depth zone is 0.889 ; the likelihood of finding the most Quinqueloculina \& Triloculina spp. tests in the Oculina Zone more than any other depth zone is 0.622 (Table 21A). The likelihood of finding the most Homotrema rubrum fragments in the Shallow Sandy Zone more than any other depth zone is 0.829 (Table 21A). The likelihood of finding the most Elphidium spp. tests in the Oculina Zone more than any other depth zone is 0.661 (Table 21A). The likelihood of finding the most Ammonia beccarii tests in the Oculina Zone more any other depth zone is 0.586 (Table 21A).

Under the medium test/fragment-size fraction, tested against temperature zones, relative effect probabilities were calculated. The likelihood of finding the most Quinqueloculina \& Triloculina spp. tests in the Subthermocline Layer more than any other temperature zone is 0.889; the likelihood of finding the most Quinqueloculina \& Triloculina spp. tests in the Thermocline Layer more than any other temperature zone is 0.556 (Table 21B); (2) the likelihood of finding the most Homotrema rubrum fragments in the Mixed Layer more than any other temperature zone is 0.565 (Table 21B). The likelihood of finding the most Elphidium spp. tests in the Mixed Layer more than any temperature zone is 0.565 (Table 21B). The likelihood of finding the most Ammonia beccarii tests in the Thermocline Layer more than any other temperature zone is 0.586 (Table 21B).

Under the medium test/fragment-size fraction, tested against salinity zones, relative effect probabilities were calculated. The likelihood of finding the most Quinqueloculina \& Triloculina spp. tests in the Euhaline Zone more than any other salinity zone is 0.750 ; the likelihood of finding the most Quinqueloculina \& Triloculina spp. tests in the Polyhaline Zone more than any other salinity zone is 0.694 (Table 21C). The likelihood of finding the most Homotrema rubrum fragments in the Mesohaline Zone more than any other salinity zone is 0.686 (Table 21C). The 
likelihood of finding the most Elphidium spp. tests in the Polyhaline Zone more than any salinity zone is 0.833 (Table 21C). The likelihood of finding the most Ammonia beccarii tests in the Polyhaline Zone more than any other salinity zone is 0.840 (Table 21C).

Under the medium test/fragment-size fraction, tested against RDO zones, relative effect probabilities were calculated. The likelihood of finding the most Quinqueloculina \& Triloculina spp. tests in the 0-2 mg/L zone more than any other RDO zone is 0.889 ; the likelihood of finding the most Quinqueloculina \& Triloculina spp. tests in the $2-4 \mathrm{mg} / \mathrm{L}$ zone more than any other RDO zone is 0.722 ; the likelihood of finding the most Quinqueloculina \& Triloculina spp. tests in the 4-6 mg/L zone more than any other RDO zone is 0.630 (Table 21D). The likelihood of finding the most Homotrema rubrum fragments in the $6-9 \mathrm{mg} / \mathrm{L}$ zone more than any other RDO zone is 0.747 (Table 21D). The likelihood of finding the most Elphidium spp. tests in the 4-6 $\mathrm{mg} / \mathrm{L}$ zone more than any RDO zone is 0.699 (Table 21D). The likelihood of finding the most Ammonia beccarii tests in the $6-9 \mathrm{mg} / \mathrm{L}$ zone more than any other $\mathrm{RDO}$ zone is 0.587 ; the likelihood of finding the most Ammonia beccarii tests in the 4-6 mg/L zone more than any other RDO zone is 0.551 (Table 21D).

Under the medium test/fragment-size fraction, tested against $\mathrm{pH}$ zones, relative effect probabilities were calculated. The likelihood of finding the most Quinqueloculina \& Triloculina spp. tests in the 7.6-7.8 zone more than any other $\mathrm{pH}$ zone is 0.889 ; the likelihood of finding the most Quinqueloculina \& Triloculina spp. tests in the 7.8-8.0 zone more than any other $\mathrm{pH}$ zone is 0.574 (Table 21E). The likelihood of finding the most Homotrema rubrum fragments in the 8.0-8.2 zone more than any other $\mathrm{pH}$ zone is 0.608 (Table 21E). The likelihood of finding the most Elphidium spp. tests in the $7.8-8.0$ zone more than any $\mathrm{pH}$ zone is 0.586 ; the likelihood of finding the most Elphidium spp. tests in the 8.0-8.2 zone more than any $\mathrm{pH}$ zone is 0.533 (Table 
21E). The likelihood of finding the most Ammonia beccarii tests in the 8.0-8.2 zone more than any other $\mathrm{pH}$ zone is 0.633 (Table $21 \mathrm{E}$ ).

\section{Fine test/fragment-size interval}

\section{Global Hypothesis}

At the fine test/fragment-size interval, four Global Hypothesis ANOVA tests were performed on the five parameters (depth, temperature, salinity, RDO, and $\mathrm{pH}$ ) using the ANOVA-Type Test, LH Test, BNP Test, and Wilks Lambda procedures. The ANOVA-Type Test for all five parameters was statistically significant ( $\mathrm{p}>0.05)$ (Table 22). The LH Test, BNP Test, and Wilks Lambda for depth, temperature, salinity, rugged dissolved oxygen also was statistically significant $(\mathrm{p}>0.05)$ (Table 22$)$. An important observation to note was that $\mathrm{pH}$ was the only environmental parameter that was not statistically significant for any tests except the ANOVAType Test (Table 22). Thus, due to the statistical significance of the Global Hypotheses for all five parameters, the Subset Algorithm for test/fragment counts was used.

\section{Subset Algorithm on fine test/fragment counts}

Following the Global Hypothesis, Subset Algorithms testing the following hypotheses: $\mathrm{H}_{0}$ : Counts for all taxa are equal; $\mathrm{H}_{\mathrm{a}}$ : At least one taxonomic group is not equal. In other words, this algorithm was used to differentiate taxa, if possible. According to Table 23, at the fine fragmentsize interval, Homotrema rubrum was considered a $1^{\text {st }}$ order, statistically distinguishable taxon for depth and salinity, and $2^{\text {nd }}$ order statistically distinguishable taxon for RDO and $\mathrm{pH}$. At the fine test-size interval, Quinqueloculina \& Triloculina spp. was considered a $2^{\text {nd }}$ order statistically distinguishable taxonomic group for depth, salinity, RDO, and $\mathrm{pH}$ (Table 23). At the fine testsize interval, Elphidium spp. was considered a $2^{\text {nd }}$ order, statistically distinguishable taxon for RDO, and statistically like Ammonia beccarii for depth, salinity, and $\mathrm{pH}$ (Table 23). At the fine test-size interval, Ammonia beccarii was $3^{\text {rd }}$ order, statistically distinguishable taxon for salinity, 
and statistically like Elphidium spp. for depth, RDO, and pH (Table 23). The two orders of statistically distinguishable taxa are categorized based on the magnitude of statistical difference from one another. For example, Homotrema rubrum was the first taxon determined by the algorithm as statistically different from the other three taxa for depth and salinity; Quinqueloculina \& Triloculina spp. was the second group determined as different for depth, salinity, RDO, and pH; Elphidium spp. was the second taxon determined as different for RDO, and Ammonia beccarii was the third taxon determined as different for salinity (Table 23).

III. Subset Algorithm on environmental parameters

The second set of Subset Algorithms tested the effects of the factor levels in each environmental parameter, under the following assumptions: $\mathrm{H}_{\mathrm{o}}$ : all factor levels in environmental parameter contributed equally to significant differences in counts. $\mathrm{H}_{\mathrm{a}}$ : at least one factor level in environmental parameter contributed differently to significant differences in counts. At the fine $\mathrm{mm}$ test/fragment-size interval, the significant differences in taxa were modeled against three depth zones: (1) Shallow Sandy Zone (0-10 m); (2) Oculina Zone (10-18 m); and (3) Subthermocline Zone (18-25 m). The Subset Algorithm for depth found the Shallow Sandy Zone as the $1^{\text {st }}$ order, statistically distinguishable zone, the Oculina Zone as the $2^{\text {nd }}$ order, statistically distinguishable zone, but found the Subthermocline zone as indistinguishable from the other two zones (Table 24). Here, the Shallow Sandy Zone was the first depth factor-level to be identified as contributing differently to significant differences in test/fragment counts. The Oculina Zone as the second factor-level to be identified as contributing to significant differences in test/fragment counts, and the Subthermocline zone was indistinguishable for contributing to significant differences in test/fragment counts (Table 24). 
When modeling the fine test/fragment-size interval against temperature, the significant differences in taxa were tested against three temperature zones: (1) Mixed Layer $\left(26-28{ }^{\circ} \mathrm{C}\right)$; (2) Thermocline Layer $\left(24-26{ }^{\circ} \mathrm{C}\right)$; and (3) Subthermocline Layer $\left(22-24{ }^{\circ} \mathrm{C}\right)($ Table 25$)$. However, the Subset Algorithm for temperature was only able to determine that at least one of these temperature zones contributed to significant differences in counts, but unable to identify which one (Table 25).

When modeling the fine test/fragment-size interval against salinity, the significant differences in taxa were tested against three salinity zones: (1) Mesohaline (2-10 psu); (2) Polyhaline (10-35 psu); and (3) Euhaline (35+ psu) (Table 26). The Subset Algorithm for salinity found the Polyhaline zone as the $1^{\text {st }}$ order, statistically distinguishable zone, but was unable to differentiate the Mesohaline zone from the Euhaline zone. Hence, the Polyhaline zone was the first salinity factor-level to be identified as contributing to significant differences in test/fragment counts.

When modeling fine test/fragment-size interval against RDO, the significant differences in taxa were tested against four RDO zones: (1) 0-2 mg/L; (2) 2-4 mg/L; (3) 4-6 mg/L; and (4) 6-9 mg/L (Table 27). The Subset Algorithm for RDO was only able to determine that at least one of these RDO zones contributed to significant differences in test/fragment counts, but unable to identify which one.

When modeling the fine fragment-size interval against $\mathrm{pH}$, the significant differences in taxa were tested against three pH zones: (1) 8.0-8.2; (2) 7.8-8.0; and (3) 7.6-7.8 (Table 28). The Subset Algorithm for $\mathrm{pH}$ found the 8.0-8.2 zone as the $1^{\text {st }}$ order, statistically distinguishable zone, but was unable to differentiate the 8.0-8.2 and 7.8-8.0 zones. Here, the 8.0-8.2 zone was 
the first $\mathrm{pH}$ factor-level to be identified as contributing to significant differences in test/fragment counts.

\section{Relative Probabilities}

Using the relative effects probability algorithms, the likelihood of test/fragment count abundance under a given factor-level for an environmental parameter was calculated for each taxon, under different test/fragment-size intervals. Under the fine test/fragment-size fraction, tested against depth zones, relative effect probabilities were calculated. The likelihood of finding the most Quinqueloculina \& Triloculina spp. tests in the Subthermocline Zone more than any other depth zone is 0.833; the likelihood of finding the most Quinqueloculina \& Triloculina spp. tests in the Oculina Zone more than any other depth zone is 0.594 (Table 29A). The likelihood of finding the most Homotrema rubrum fragments in the Shallow Sandy Zone more than any other depth zone is 0.815 (Table 29A). The likelihood of finding the most Elphidium spp. tests in the Oculina Zone more than any other depth zone is 0.597 (Table 29A). The likelihood of finding the most Ammonia beccarii tests in the Subthermocline Zone more any other depth zone is 0.944; the likelihood of finding the most Ammonia beccarii tests in the Oculina Zone more any other depth zone is 0.506 (Table 29A).

Under the fine test/fragment-size fraction, tested against temperature zones, relative effect probabilities were calculated. The likelihood of finding the most Quinqueloculina \& Triloculina spp. tests in the Subthermocline Layer more than any other temperature zone is 0.833 (Table 29B). The likelihood of finding the most Homotrema rubrum fragments in the Thermocline Layer more than any other temperature zone is 0.563 ; the likelihood of finding the most Homotrema rubrum fragments in the Mixed Layer more than any other temperature zone is 0.535 (Table 29B). The likelihood of finding the most Elphidium spp. tests in the Mixed Layer more than any temperature zone is 0.539 (Table 29B). The likelihood of finding the most 
Ammonia beccarii tests in the Subthermocline Layer more than any other temperature zone is 0.944; the likelihood of finding the most Ammonia beccarii tests in the Thermocline Layer more than any other temperature zone is 0.729 (Table 29B).

Under the fine test/fragment-size fraction, tested against salinity zones, relative effect probabilities were calculated. The likelihood of finding the most Quinqueloculina \& Triloculina spp. tests in the Polyhaline Zone more than any other salinity zone is 0.764 ; the likelihood of finding the most Quinqueloculina \& Triloculina spp. tests in the Euhaline Zone more than any other salinity zone is 0.736 (Table 29C). The likelihood of finding the most Homotrema rubrum fragments in the Mesohaline Zone more than any other salinity zone is 0.714 (Table 29C). The likelihood of finding the most Elphidium spp. tests in the Polyhaline Zone more than any salinity zone is 0.889 . The likelihood of finding the most Ammonia beccarii tests in the Euhaline Zone more than any other salinity zone is 0.799 .

Under the fine test/fragment-size fraction, tested against RDO zones, relative effect probabilities were calculated. The likelihood of finding the most Quinqueloculina \& Triloculina spp. tests in the $0-2 \mathrm{mg} / \mathrm{L}$ zone more than any other RDO zone is 0.833 ; the likelihood of finding the most Quinqueloculina \& Triloculina spp. tests in the 4-6 mg/L zone more than any other RDO zone is 0.662 (Table 29D). The likelihood of finding the most Homotrema rubrum fragments in the 6-9 $\mathrm{mg} / \mathrm{L}$ zone more than any other RDO zone is 0.681 ; the likelihood of finding the most Homotrema rubrum fragments in the 2-4 mg/L zone more than any other RDO zone is 0.528 . The likelihood of finding the most Elphidium spp. tests in the $4-6 \mathrm{mg} / \mathrm{L}$ zone more than any RDO zone is 0.579 . The likelihood of finding the most Ammonia beccarii tests in the 0$2 \mathrm{mg} / \mathrm{L}$ zone more than any other RDO zone is 0.944 ; the likelihood of finding the most Ammonia beccarii tests in the $2-4 \mathrm{mg} / \mathrm{L}$ zone more than any other $\mathrm{RDO}$ zone is 0.806 ; the 
likelihood of finding the most Ammonia beccarii tests in the 4-6 mg/L zone more than any other $\mathrm{RDO}$ zone is 0.537 .

Under the fine test/fragment-size fraction, tested against $\mathrm{pH}$ zones, relative effect probabilities were calculated. The likelihood of finding the most Quinqueloculina \& Triloculina spp. tests in the 7.6-7.8 zone more than any other pH zone is 0.833 (Table 29E). The likelihood of finding the most Homotrema rubrum fragments in the 8.0-8.2 zone more than any other $\mathrm{pH}$ zone is 0.556 ; the likelihood of finding the most Homotrema rubrum fragments in the 7.8-8.0 zone more than any other $\mathrm{pH}$ zone is 0.519 . The likelihood of finding the most Elphidium spp. tests in the 8.0-8.2 zone more than any $\mathrm{pH}$ zone is 0.567 . The likelihood of finding the most Ammonia beccarii tests in the 7.6-7.8 zone more than any other $\mathrm{pH}$ zone is 0.944 ; the likelihood of finding the most Ammonia beccarii tests in the 7.8-8.0 zone more than any other $\mathrm{pH}$ zone is 0.704 .

\section{Very fine test/fragment-size interval}

V. Global Hypothesis

At the very fine test/fragment-size interval, four Global Hypothesis ANOVA tests were performed on the five parameters (depth, temperature, salinity, $\mathrm{RDO}$, and $\mathrm{pH}$ ) using the ANOVA-Type Test, LH Test, BNP Test, and Wilks Lambda procedures. The ANOVA-Type Test for all five parameters was statistically significant ( $p>0.05)$ (Table 30). The ANOVA-Type Test, LH Test, BNP Test, and Wilks Lambda for depth, salinity, and pH also were statistically significant. An important observation to note was that temperature and $\mathrm{pH}$ were the only environmental parameters that were not statistically significant for any tests (Table 30). Thus, due to the statistical significance of the Global Hypotheses for all five parameters, using at least one significant test, the Subset Algorithm for test/fragment counts proceeded for depth, salinity, and $\mathrm{pH}$. 
VI. Subset Algorithm on very fine test/fragment counts

Following the Global Hypothesis, Subset Algorithms tested the following hypotheses: $\mathrm{H}_{\mathrm{o}}$ : Counts for all taxa are equal; $\mathrm{H}_{\mathrm{a}}$ : At least one taxonomic group is not equal. In other words, this algorithm was used to differentiate the taxa, if possible. According to Table 31, at the very fine fragment-size interval, Homotrema rubrum was considered a $1^{\text {st }}$ order, statistically distinguishable taxon for salinity, and statistically similar to Quinqueloculina \& Triloculina spp. for depth. At the very fine test-size interval, Quinqueloculina \& Triloculina spp. was considered a $2^{\text {nd }}$ order statistically distinguishable taxonomic group for salinity, and statistically similar to Homotrema rubrum for depth. At the very fine test-size interval, Elphidium spp. was considered a $1^{\text {st }}$ order, statistically distinguishable taxon for depth, and statistically like Ammonia beccarii for salinity. At the very fine test-size interval, Ammonia beccarii was $2^{\text {nd }}$ order, statistically distinguishable taxon for depth, and statistically similar to Elphidium spp. for salinity. The two orders of statistically distinguishable taxa are categorized based on the magnitude of statistical difference from one another. For example, Homotrema rubrum was the first taxon determined by the algorithm as statistically different from the other three taxa for salinity, Quinqueloculina \& Triloculina spp. was the second group determined as different for salinity, Elphidium spp. was the first taxon determined as different for depth, and Ammonia beccarii was the second taxon determined as different for depth. It was also important to note that the statistical distinguishing procedure was limited to depth and salinity because temperature and RDO were insignificant from the Global Hypothesis, and the algorithm was unable to determine statistical differences for pH (Table 31). 
VII. Subset Algorithm on environmental parameters

The second set of Subset Algorithms tested the effects of the factor levels in each environmental parameter, under the following assumptions: $\mathrm{H}_{\mathrm{o}}$ : All factor levels in environmental parameter contributed equally to significant differences in counts. $\mathrm{H}_{\mathrm{a}}$ : At least one factor level in environmental parameter contributed to significant differences in counts. At the very fine test/fragment-size interval, the significant differences in taxa were modeled against three depth zones: (1) Shallow Sandy Zone (0-10 m); (2) Oculina Zone (10-18 m); and (3) Subthermocline Zone (18-25 m). The Subset Algorithm for depth found the Shallow Sandy Zone as the $1^{\text {st }}$ order, statistically distinguishable zone, the Oculina Zone as the $2^{\text {nd }}$ order, statistically distinguishable zone, but found the Subthermocline zone as indistinguishable from the other two zones (Table 32). Hence, the Shallow Sandy Zone was the first depth factor-level to be identified as contributing differently to significant differences in test/fragment counts, the Oculina Zone as the second factor-level to be identified as contributing differently to significant differences in test/fragment counts, and the Subthermocline zone was indistinguishable for contributing to significant differences in test/fragment counts.

When modeling the very fine test/fragment-size interval against temperature, the significant differences in taxa were tested against three temperature zones: (1) Mixed Layer $\left(26-28^{\circ} \mathrm{C}\right)$; (2) Thermocline Layer $\left(24-26^{\circ} \mathrm{C}\right)$; (3) Subthermocline Layer $\left(22-24{ }^{\circ} \mathrm{C}\right)$ (Table 33). However, the Subset Algorithm for temperature was not performed because the Global Hypothesis was not statistically significant (Table 33).

When modeling the very fine test/fragment-size interval against salinity, the significant differences in taxa were tested against three salinity zones: (1) Mesohaline (2-10 psu); (2) Polyhaline (10-35 psu); and (3) Euhaline (35+ psu) (Table 34). The Subset Algorithm for salinity 
found the Mesohaline zone as the $1^{\text {st }}$ order, statistically distinguishable zone, the Polyhaline zone as the $2^{\text {nd }}$ order, statistically distinguishable zone, and the Euhaline zone as statistically indistinguishable from the other two zones (Table 34). Here, the Mesohaline zone was the first salinity factor-level to be identified as contributing to significant differences in test/fragment counts, while the Polyhaline zone was the second salinity factor-level to be identified as contributing to significant differences in test/fragment counts.

When modeling the very fine test/fragment-size interval against RDO, the significant differences in taxa were tested against four RDO zones: (1) 0-2 mg/L; (2) 2-4 mg/L; (3) 4-6 $\mathrm{mg} / \mathrm{L}$; and (4) 6-9 mg/L (Table 35). The Subset Algorithm for RDO was not performed because the Global Hypothesis was not statistically significant (Table 35).

When modeling the very fine test/fragment-size interval against $\mathrm{pH}$, the significant differences in taxa were tested against three $\mathrm{pH}$ zones: (1) 8.0-8.2; (2) 7.8-8.0; and (3) 7.6-7.8 (Table 36). The Subset Algorithm for $\mathrm{pH}$ was only able to determine that at least one $\mathrm{pH}$ zone contributed to significant differences in counts but was unable to determine which one.

VIII. Relative Probabilities

Using the relative effects probability algorithms, the likelihood of test/fragment count abundance under a given factor-level for an environmental parameter was calculated for each taxon, under different test/fragment-size intervals. Under the very fine test/fragment-size fraction, tested against depth zones, relative effect probabilities were calculated. The likelihood of finding the most Quinqueloculina \& Triloculina spp. tests in the Oculina Zone more than any other depth zone is 0.636 (Table 37A). The likelihood of finding the most Homotrema rubrum fragments in the Subthermocline Zone more than any other depth zone is 0.875 ; the likelihood of finding the most Homotrema rubrum fragments in the Shallow Sandy Zone more than any other depth zone is 0.597 . The likelihood of finding the most Elphidium spp. tests in the Oculina Zone 
more than any other depth zone is 0.669 ; the likelihood of finding the most Elphidium spp. tests in the Subthermocline Zone more than any other depth zone is 0.597 . The likelihood of finding the most Ammonia beccarii tests in the Shallow Sandy Zone more any other depth zone is 0.824 .

Under the very fine test/fragment-size fraction, tested against salinity zones, relative effect probabilities were calculated. The likelihood of finding the most Quinqueloculina \& Triloculina spp. tests in the Polyhaline Zone more than any other salinity zone is 0.875 (Table 37B). The likelihood of finding the most Homotrema rubrum fragments in the Euhaline Zone more than any other salinity zone is 0.799 ; the likelihood of finding the most Homotrema rubrum fragments in the Polyhaline Zone more than any other salinity zone is 0.536 . The likelihood of finding the most Elphidium spp. tests in the Polyhaline Zone more than any salinity zone is 0.847 ; the likelihood of finding the most Elphidium spp. tests in the Euhaline Zone more than any salinity zone is 0.535 . The likelihood of finding the most Ammonia beccarii tests in the Mesohaline Zone more than any other salinity zone is 0.636 .

Under the very fine test/fragment-size fraction, tested against $\mathrm{pH}$ zones, relative effect probabilities were calculated. The likelihood of finding the most Quinqueloculina \& Triloculina spp. tests in the 8.0-8.2 zone more than any other $\mathrm{pH}$ zone is 0.592 (Table 37C). The likelihood of finding the most Homotrema rubrum fragments in the 7.6-7.8 zone more than any other $\mathrm{pH}$ zone is 0.875 ; the likelihood of finding the most Homotrema rubrum fragments in the 7.8-8.0 zone more than any other $\mathrm{pH}$ zone is 0.537 . The likelihood of finding the most Elphidium spp. tests in the 7.6-7.8 zone more than any $\mathrm{pH}$ zone is 0.597 ; the likelihood of finding the most Elphidium spp. tests in the 7.8-8.0 zone more than any $\mathrm{pH}$ zone is 0.551 . The likelihood of finding the most Ammonia beccarii tests in the 8.0-8.2 zone more than any other $\mathrm{pH}$ zone is 0.633 . 


\section{$\underline{\text { Ordination }}$}

\section{Non-metric Multidimensional Scaling}

After performing 20 trials, the nMDS iterative algorithm produced a Shephard stress plot, illustrating the relationship between the actual dissimilarities of the taxa from the prenMDS matrix and the ordinal distances scaled by the Bray algorithm (Figure 16). Because these two variables were highly correlated, the nMDS underwent 20 trials and yielded two convergent solutions, under two dimensions, with a stress value of 0.017 (Table 38). Thus, with a stress value less than 0.05 , the nMDS produced a trustworthy dissimilarity matrix that can ultimately be plotted for interpretation.

When plotted in ordinal space, results were interpreted by relative distance, where data points ordinated closer together were interpreted as more similar (Figure 17). The ordinal diagram shows that all test/fragment-size fractions of Homotrema rubrum, Quinqueloculina \& Triloculina spp., and Elphidium spp. were moderately-to-highly concentrated around one another, logically grouping the different test/fragment-size intervals of each taxon together (Figure 17). Unlike these three taxa, test-size fractions of Ammonia beccarii were scattered far apart, where fine and very fine test-size fractions ordinated closely to fine and very fine fragment-size fractions of Homotrema rubrum, and the medium test-size fraction of Ammonia beccarii ordinated closely with all three test-size fractions of Elphidium spp (Figure 17). Thus, although most test/fragment-size fractions of each taxon were consistently ordinated around similar groupings, there is evidence of unusual dissimilarities.

\section{$\underline{\text { Analysis of Similarities (ANOSIM) }}$}

Five ANOSIM tests and diagrams were produced, each modeling the ordinal values of Quinqueloculina \& Triloculina spp., Homotrema rubrum, Elphidium spp., and Ammonia beccarii against all five environmental parameters (depth, temperature, salinity, RDO, $\mathrm{pH}$ ). The 
two things that are most important when assessing an ANOSIM diagram are the R-value and the p-value. R-values determine whether there is dissimilarity among the factor levels of environmental parameters, within the factor levels, or an even distribution of dissimilarity between and within ranks. P-values dictate the statistical significance of these R-values. R-values closer to 1 suggest dissimilarity between factor levels, R-values closer to 0 suggest an even distribution of dissimilarity between factor levels and within each factor level, and R-values below 0 suggest dissimilarity within each factor level.

In the ANOSIM diagram of depth, the R-value was 0.586 , which was statistically significant with a p-value of 0.001 , ultimately indicating moderate dissimilarity between the different depth zones (Figure 18A). In the boxplots, there were two noteworthy observations to be made: (1) there appeared to be a disparity of range among the different depth zones; and (2) the box representing dissimilarity between the zones ("Between") significantly differed from the remaining boxes (Figure 18A). Based on the moderate-to-moderately high R-value, the boxplot representing dissimilarity between the depth zones accurately correspond the dissimilarity magnitude.

In the ANOSIM diagram of temperature, the R-value was 0.122 , which was statistically insignificant with a p-value of 0.165 . Hence, there is no difference between the temperature zones (Figure 18B). Furthermore, due to the statistical insignificance, interpretive procedures were not continued and performed.

In the ANOSIM diagram of salinity, the R-value was 0.486 , which was statistically significant with a p-value of 0.001 , suggesting close-to-moderate dissimilarity among the different salinity zones (Figure 18C). To a lesser magnitude when compared to the ANOSIM diagram of depth, the ANOSIM diagram of salinity exhibited less disparity among the boxplot 
ranges, although, the box representing the dissimilarities between the salinity zones moderately differed from the other boxes (Figure 18C). Thus, based on the moderate R-value, the dissimilarity box's slight deviation from the other salinity zone boxes accurately captured the magnitude of dissimilarity.

In the ANOSIM diagram of RDO, the R-value was 0.471 , which was statistically significant with a p-value of 0.001 , suggesting close to moderate dissimilarity among the RDO zones. This result is similar to the ANOSIM diagram of depth (Figure 18D). There were two noteworthy observations: (1) the disparity between the boxplots of the RDO zones were drastic, with Hypoxic to Anoxic (0-2 mg/L) and 22-44\% (2-4 mg/L) being tiny boxplots with little-to-no variability; and (2) the displacement of the box representing dissimilarity between the RDO zones was less than what was seen in the ANOSIM diagrams of depth and salinity (Figure 18D). Thus, based on a moderate R-value, the dissimilarity magnitude agreed with the different array of boxes.

In the ANOSIM diagram of $\mathrm{pH}$, the R-value was 0.267 , which was statistically significant with a p-value of 0.017 , suggesting a lack of dissimilarity between the different $\mathrm{pH}$ zones (Figure 18E). Here, the drastic differences between the tiny box for the 7.6-7.8 zone and boxes of the other zones were noteworthy; however, the dissimilarity boxplot was not distinguishable from the 8.0-8.2 $\mathrm{pH}$ zone. Furthermore, despite the differences in boxplot shapes, the dissimilarity represented by the boxplot and the R-value indicated that there was strong similarity between $\mathrm{pH}$ zones and perhaps an increased level of dissimilarity within each $\mathrm{pH}$ zone.

\section{Geospatial Analysis}

\section{Additive Quantile Regression Smoothing}

Using a smoothing parameter of 0.01 and 100 artificial vertices, Figure 19 was produced with a color gradient with corresponding factor-level ranges. In the depth AQR map, there were 
three noteworthy observations. First, depth ranges between 19 and 25 meters were concentrated in the region between sampling sites 7 and 10, which resembled Patton's Basin, bordering the northern section of Devil's Hole. Second, shallower depth ranges from 0 and 10 meters were concentrated in the northern and eastern part of the spatial map, around Trunk Island and Middle Ridge. And third, there seems to be a depth gradient, where depth decreased as it fanned out from the sampling site 10 (Figure 19A). Following the trends in the depth AQR map, the temperature and RDO AQR maps illustrated similar trajectories, where both temperature and RDO decreased towards sampling site 10 and increased as it radiated out from that centerpoint (Figure 19B and Figure 19D). In contrast, AQR maps of salinity and $\mathrm{pH}$ differed from these patterns, where there appears to be salinity and $\mathrm{pH}$ zones (Figure 19C, Figure 19E). In the salinity AQR map, there were two contoured, salinity zones that ranged from 10 to 37.5 psu: (1) region around sampling sites $1,3,5$, and 7; and (2) the southern and eastern contoured regions that included sampling sites 2, 8, and 10 (Figure 19C). In the pH AQR map, higher pH (8.0-8.2) appears to be concentrated in the central portion of the map that included sampling sites 1,3 , and 7, and lower in $\mathrm{pH}$ as it radiaed out from the central portion (Figure 19E). Thus, from mapping the predictive results seen from quantile regression modeling, these spatial maps illustrated unique trajectories that profile analysis was not able to capture.

\section{ArcMap TIN and Surface Contour}

Using ArcMap's Triangulated Irregular Networks (TIN) tool, contoured trajectories of specific test/fragment-size fractions of the four taxa (Quinqueloculina \& Triloculina spp., Homotrema rubrum, Elphidium spp., and Ammonia beccarii) were plotted over spatial maps of the five environmental parameters (depth, temperature, salinity, $\mathrm{RDO}, \mathrm{pH}$ ). Unlike $\mathrm{AQR}$ smoothing seen in the previous section, ArcMap's TIN function used triangulated interpolation to produce the spatial maps of the parameters. This resulted in both similar and slight differences 
in patterns and trends. Additionally, the water-chemistry measurements around the major cave passageways and Flatt's Inlet taken on July $1^{\text {st }}, 2018$, were added to expand the interpolation process and spatial maps (Figure 20).

\section{Environmental parameters}

The depth TIN map appears to show similar-to-identical trajectories to the depth AQR map, where the region around sampling sites 7 and 10 was the deepest and depth decreased fanning out from that center point (Figure 20A). Correspondingly, the temperature and RDO TIN maps illustrate the same relationship between depth, temperature, and RDO as seen in the AQR maps. Lower temperature and RDO ranges were concentrated around sampling sites 7 and 10 and increased away from those sites (Figures 20B and 20C). In contrast, salinity and pH TIN maps are slightly more intricate with specific salinity and $\mathrm{pH}$ zones and patterns. There are three contoured zones in the salinity TIN map that ranged from 10 to $37.5 \mathrm{psu}$ : (1) the area surrounding sampling sites 5 and 6 ; (2) the southern portion of the map that included sampling site 10; and (3) the northeastern portion of the spatial map that included sampling site 2 (Figure 20C). Additionally, areas with significantly higher salinity levels include the western edge of the map, which was surrounding the Green Bay caves, and the southwestern portion, which characterized the Flatt's Inlet opening (Figure 20C). In the pH TIN map, there is a large, centralized area with higher $\mathrm{pH}$ ranges (8.0-8.2), which followed the scatter of every sampling site except sites 7 and 10 (Figure 20E). Besides this centralized area, the pH tended to decrease towards the margins of the basin (Figure 20E). Thus, despite the methodological differences between AQR and TIN mapping, the similarities in trajectory and trends assure data consistency and spatial-analytical compatibility. 


\section{Quintueloculina \& Triloculina spp.}

I. Coarse test-size interval

When the calculated contours of the coarse test-size fraction of Quintueloculina \&

Triloculina spp. are overlain on top of the environmental spatial maps, there were discernable observations among all five environmental parameters. The contour intervals showed that: (1) test counts increased south of sampling site 1 and east of sampling sites 4 and 6; and (2) higher test counts appeared to concentrate around sites 7, 8, and 10, which corresponded with increased depth, decreased temperature, decreased RDO, and decreased pH (Figure 20A, 20B, 20D, and 20E). In contrast, there does not appear to be a discernable, spatial relationship between salinity and test-counts of the coarse test-size fraction of Quintueloculina \& Triloculina spp., where high test counts were concentrated in both high and low salinity regions (Figure 20C).

\section{Medium test-size interval}

Similar to the coarse test-size fraction of Quintueloculina \& Triloculina spp., the medium test-size fraction of Quintueloculina \& Triloculina spp. illustrated similar-to-identical trajectories. Higher test counts are concentrated south of sampling site 1, east of sampling sites 4 and 6, and southwest of sampling site 8 (Figure 21B). Additionally, this distribution correlated with increased depth, decreased temperature, decreased RDO, and decreased $\mathrm{pH}$. The same pattern was seen in the coarse test-size interval results (Figure 21). Furthermore, there continues to be no recognizable relationship between test counts and salinity. Despite these similarities, the range of variability of these counts was different from the previous test-size fraction, where the coarse test-size fraction exhibited a range from 40 to 80 and the medium test-size fraction exhibited a range from 40 to 60 (Figure 21). 


\section{Fine test-size interval}

In the fine test-size fraction of Quintueloculina \& Triloculina spp., there were drastic changes in the number of calculated contour boundaries, range of variability in test counts, and contour patterns when compared to the previous two test-size fractions (Figure 22). The contoured trajectory shows that test counts increase towards the northeast, where moving northeast along the transect exhibits a constant increase in test counts. The highest test-count contour lines surrounded sampling site 2 . In environmental terms, high test-counts only correlated with increased depth, while there was an absence of any discernable relationship with temperature, salinity, RDO, and $\mathrm{pH}$. In addition to a lack of correlation, the number of contour boundaries (13 $\rightarrow 13 \rightarrow 10)$ and range of variability in the fine test-size fraction decreased (40$80 \rightarrow 40-60 \rightarrow 34-46)$ when compared to the previous two test-size fractions.

IV. Very fine test-size interval

The very fine test-size fraction of Quinqueloculina \& Triloculina spp. exhibited similar trends to the fine size-fraction, regarding the decrease in contour boundaries $(13 \rightarrow 13 \rightarrow 10 \rightarrow$ $5)$ and range of variability $(40-80 \rightarrow 40-60 \rightarrow 34-46 \rightarrow 22-30)$ (Figure 23$)$. The test counts linearly increased towards the northeast from sampling sites 4, 6 and 10 to sites 2 and 8 (Figure 23). Furthermore, due to a reduced number of contour boundaries, there is no discernable relationship between test counts and any of the five environmental parameters, unlike the previous larger test-size fractions (Figure 23).

\section{Homotrema rubrum}

I. Coarse fragment-size interval

In the coarse fragment-size fraction of Homotrema rubrum, the contour intervals showed that: (1) test counts increased towards sampling site 1 and sampling sites 4 and 6; and (2) higher test counts appears to concentrate around sites 1,3, and 5. These relationships correspond with 
decreased depth, increased temperature, decreased salinity, increased RDO, and increased $\mathrm{pH}$

(Figure 24). It appears that high-test count abundance of the coarse size-fraction is concentrated in areas where lower test-count concentrations of the coarse test-size fraction of Quinqueloculina \& Triloculina spp occur, and vice versa. Furthermore, due to these inverse relationships between test-count abundances of the coarse test-size fraction of Quinqueloculina \& Triloculina spp and fragment-count abundance of coarse size-fraction of $H$. rubrum, the relationship between count abundances and environmental trajectories also was found to be reversed between the two taxa.

II. Medium fragment-size interval

Similar to the coarse fragment-size fraction of H. rubrum, the medium fragment-size fraction shows a similar-to-identical trajectory. Higher test counts are concentrated towards sampling sites 1 and 4 (Figure 25). This trend corresponds with decreased depth, decreased temperature, decreased salinity, increased RDO, and increased $\mathrm{pH}$, and is similar to the coarse fragment-size interval results. Furthermore, the range of variability of these counts shows little differences from the previous fragment-size interval, where the coarse fragment-size fraction exhibited a range from 20 to 60 and the medium fragment-size fraction exhibited a range from 20 to 50 .

III. Fine fragment-size interval

There are changes in the patterns of contour boundaries and range of variability in fragment counts of the fine fragment-size fraction of H. rubrum, when compared to the previous two testsize fractions (Figure 26). The contours continue to parallel the trend seen in the previous two fragment-size intervals, where test counts increased towards sampling site 1 . However, there was differences in contour definition among certain regions in the spatial map. These include the southwestern portion around sites 4 and 6, where fewer interpolated contour lines occur when compared to the previous fragment-size intervals, and the northwestern portion surrounding sampling site 5, which became an isolated, fragment-count zone (Figure 26). In environmental 
terms, high test counts continued to correlate with decreased depth, increased temperature, decreased salinity, increased RDO, and increased $\mathrm{pH}$ (Figure 26). Additionally, the range of variability of these counts narrowed when compared to the previous fragment-size intervals (20$60 \rightarrow 20-50 \rightarrow 27-45)$.

IV. Very fine fragment-size interval

In the very fine fragment-size fraction of H. rubrum, there were fewer contour boundaries $(13 \rightarrow 13 \rightarrow 13 \rightarrow 8)$ and a change in the range of variability $(20-60 \rightarrow 20-50 \rightarrow 27-45 \rightarrow 30-$ 38) (Figure 27). The contour trajectory decreased in complexity and changed in orientation, where high test-counts concentrated around sampling sites $1,3,5,6$, and 7, and fragment counts generally increased towards the Sound's center from sampling sites 2 and 8 (Figure 27). In terms of complexity, contours were more widely spaced, which led to the overall high-test zonation and decreased complexity (Figure 27). Furthermore, despite the decreased complexity of count trajectory, there remained a weak but discernable relationship between test counts and several environmental parameters. High test-counts corresponded with increased depth, increased temperature, increased RDO, and increased $\mathrm{pH}$. Unlike the previous fragment-size fractions of H. rubrum, fragment counts of the very fine size-fraction demonstrate no relationship with salinity.

\section{Elphidium spp.}

I. Medium test-size interval

In the medium test-size fraction of Elphidium spp., contour trajectory, number of contours, and range of variability in test counts were significantly different from the other taxa. In terms of contour patterns, a concentration of contour boundaries occurs around sampling sites 1, 3, 5, 6, and 7, which also happened to be where high-test counts are concentrated (Figure 28). However, the range of variability remains very small, ranging from 5 to 10 test counts, unlike the 
test/fragment-size fractions of the other taxa. Furthermore, due to this small range of variability, ArcMap's TIN function was unable to interpolate contour boundaries in the area east of sampling sites $1,3,4$, and 7 . In terms of environmental trends, this pattern corresponded with increased temperature and increased RDO, but there is no relationship with depth, salinity, and $\mathrm{pH}$.

\section{Fine test-size interval}

Similar to the medium test-size fraction of Elphidium spp., the fine test-size fraction exhibits similar contour trajectories, where contour boundaries are concentrated around sampling sites 1 , 3, 5, 6, and 7, with major spacing in the center of the contour map (Figure 29). In addition to this contour zone, there appears to be an increasing test-count trend towards sampling site 2 . However, there was few contour boundaries showing the trend of increased test-counts. Again, the range of variability is very limited, with test counts ranging from 6 to 12 . In terms of environmental correlation, the only parameters that appears to correlate with the contour trajectories were increased temperature and RDO.

\section{Very fine test-size interval}

When compared to the previous two test-size fractions of Elphidium spp., the very fine testsize fraction exhibits dramatic differences in contour patterns and correlations with environmental factors (Figure 30). The contour trajectory shows that high-test counts are concentrated both around sampling sites $1,3,5,6$, and 7, and towards sampling sites 2, 8, and 10 in the eastern and southeastern portions of the map, respectively (Figure 30). Additionally, there are significantly fewer gaps in contours, indicating that ArcMap's TIN function was able to interpolate more contour boundaries due to the increased range of variability compared to the previous test-size fractions $(5-10 \rightarrow 6-12 \rightarrow 4-14)$. In term of correlation to environmental factors, high test-counts correlated with increased depth, increased temperature, increased salinity, increased RDO, while a moderate-to-strong relationship with $\mathrm{pH}$ is not found. Thus, it 
appears that as test-size intervals decreased, test-counts of Elphidium spp. increased in contour complexity, decreased contour gaps, increased range of variability, and increased correlation with environmental factors.

\section{$\underline{\text { Ammonia beccarii }}$}

I. Medium test-size interval

The contour trajectory in the medium test-size fraction of Ammonia beccarii is similar to the medium and fine test-size fractions of Elphidium spp.. Here, there is a concentration of high-test counts surrounding sampling sites 1,3,5,6, and 7. Major gaps of contour boundaries occur in the center of the contour map (Figure 31). Additionally, test counts increased towards sampling site 4 in the southwest and towards sampling site 2 in the northeast. Like the test-size fractions of Elphidium spp., the range of variability for the medium size-fraction of Ammonia beccarii was very limited, ranging from 5 to 10 test counts. When correlated with environmental parameters, high test-counts correspond with increased temperature, increased $\mathrm{RDO}$, and increased $\mathrm{pH}$, but no discernable relationship exists with depth and salinity. Thus, with a limited range of variability, there were major gaps in contour boundaries and an absence of correlation with certain parameters.

\section{Fine test-size interval}

Drastically different from the medium test-size fraction of $A$. beccarii, the fine test-size fraction shows a completely different contour pattern. There is a decreased number of contour boundaries $(14 \rightarrow 4)$, an increased range of variability $(5-10 \rightarrow 16-22)$, and increased correlation with environmental parameters (Figure 32). The contour trajectory shows that high test-counts are concentrated towards sampling site 10 in the south, unlike what was seen in the medium testsize fraction. High test-counts correlate with increased depth, decreased temperature, decreased $\mathrm{RDO}$, and decreased $\mathrm{pH}$, but there is no recognizable relationship with salinity (Figure 32). Thus, 
despite the decrease in contour boundaries, the range of variability in test counts significantly increased and spatial correlations to environmental parameters also strengthened.

\section{Very fine test-size interval}

Unlike either of the previous test-size fractions of $A$. beccarii, the very fine test-size fraction shows very different contour trajectories, increased distributive test-counts $(5-10 \rightarrow 16-22 \rightarrow 24-$ 30), and changes in environmental influences (Figure 33). The contour pattern is the opposite trend as seen in the fine test-size fraction, where test counts increased towards sampling sites 1 and 3. High test-counts correlate with decreased depth, increased temperature, decreased salinity, increased RDO, and increased $\mathrm{pH}$. Hence, there is a strong spatial correlation with all five environmental parameters. Furthermore, in addition to the changes in test-count trajectory and range of variability, test counts for the very fine test-size fraction shows increased correlation with environmental factors.

\section{DISCUSSION}

\section{Environmental parameters}

The depth distribution illustrated in Figure 4A demonstrates a strong, inverse correlation with temperature, rugged dissolved oxygen, and $\mathrm{pH}$. However, there is little-to-no relationship between depth and salinity. Sampling sites $2,5,6$, and 10 possessed distinctly higher salinityvalues in the polyhaline and euhaline ranges, illustrating the presence of a salt-water input or a connection to the open ocean. Due to the proximity to Green Bay Cave, which exhibited predominantly euhaline values at the opening in Harrington Sound (Figure 5C), sites 5 and 6 possess similar salinities (Figure 4B). These high salinity values indicate a significant exchange with marine waters, which is accurate because Green Bay Cave exchanges directly with openoceanic waters (Figure 2); (Stoffer and Iliffe, 2013). Due to the proximity to the isthmus between Harrington Sound and Castle Harbour, site 2 reflects a similar salinity as Castle Harbour (34-37 
psu) and its exchange with the Sound (Figure 2 and Figure 4C); (Stoffer and Iliffe, 2013). Unlike the previous two sites, sampling site 10 was not located near any connection to open marine waters; yet, it exhibits salinity values characteristic of oceanic waters. However, site 10 is near Devil's Hole, the deepest part of the Bermuda pedestal comprised of submerged caves. According to Stoffer and Iliffe (2013), salinity values at Devil's Hole vary between 34-35 psu. Therefore, the high salinity values seen at site 10 may also the result of saltwater input, which was seen in sites 2,5 , and 6 .

$\underline{\text { Environmental parameters along caves and Flatt's Inlet with seasonal differences }}$

Physical and geochemical measurements taken at the cave openings and Flatt's Inlet in the summer of 2018 and the winter of 2019 are similar in variability. However, the values reflect differences in seasonality (Figures 5 and 6). Due to the increased number of samples taken in January of 2019 versus those in July of 2018, depth measurements taken around Harrington Sound were above 10 meters, which contributed to the overall depth range, whereas the summer samples only had depths up to 6 meters (Figures 5A and 6A).

Temperature values showed little-to-no fluctuations across all sites, but the average temperatures in July vs January were drastically different $\left(25-30{ }^{\circ} \mathrm{C} \rightarrow 15-20{ }^{\circ} \mathrm{C}\right.$ ) (Figures $5 \mathrm{~B}$ and 6B). This dramatic drop could be explained by seasonality, where water temperatures logically drop during the winter and rise during the summer.

Salinity showed drastic changes both in variability and average values between the summer and winter samples. Summer measurements reflect a salt-water influence, with a range between mesohaline and euhaline psu values. Though, after reviewing these values against previous studies, there may have been an instrumental malfunction that might have inaccurately measured mesohaline values in Harrington Sound. Winter measurements show little-to-no 
variability and all were euhaline (Figures 5C and 6C). These changes could be explained by the increased likelihood of storms and inclement weather during the winter. These would increase and exacerbate both the exchange between Harrington Sound and fully marine waters, as well as water-column mixing, both of which would result in a more isoclinal range of values.

Both summer and winter RDO measurements show minimal variability in values, but the summer samples were lower on average $(5.5-7.5 \rightarrow 6.9-7.9)$ (Figures 5D and 6D). In the production of dissolved oxygen content, warmer waters generally promote increased organic matter and biogeochemical activity, which would result in lower RDO values. In contrast, colder waters generally possess less organic and biogeochemical inputs, resulting in higher RDO values (Pohlman, 2011). Additionally, due to the increased mixing in the winter water-column, RDO values were subjected to a similar mixing process. Nevertheless, due to decreased temperatures between July and January, the dissolved oxygen content decreased from summer to winter.

The variability of $\mathrm{pH}$ in both the July and the January samples were similar, where there was little-to-no change across the sampling sites. However, the average $\mathrm{pH}$ between the two timelines was dramatically different $(7.7 \rightarrow 8.2$ ) (Figures $5 \mathrm{E}$ and $6 \mathrm{E})$. Here, the trajectory was comparative to RDO, which has been correlative with $\mathrm{pH}$ in previous studies (Stoff and Iliffe, 2013). Hence, increased organic matter content and biogeochemical processes lowers RDO, and the hydrogen sulfide produces lower pH values, as well (Fairfield et al., 2009). Thus, correlative with dissolved oxygen trends, $\mathrm{pH}$ values in Harrington Sound were lower during periods of higher temperatures, increased organic matter, and lower RDO, and vice versa. 


\section{$\underline{\text { Test/fragment counts }}$}

\section{Quinqueloculina \& Triloculina spp.}

The relationships illustrated in the test/fragment count distributions show correlations between test/fragment-size fractions, overall test-count average, and range of variability. Figures 8-11 illustrate that Quinqueloculina \& Triloculina spp. test counts and Homotrema rubrum fragment counts decreased in both count averages and range of variability as test-size fraction decreased. In contrast, Elphidium spp. and Ammonia beccarii test counts increased in both testcount averages and range of variability. The patterns seen in the test-size fractions of Quinqueloculina \& Triloculina spp. could be explained by the sampling sites' proximity to karst platforms and nearshore locations, where miliolid foraminifera generally concentrate (Neumann, 1965). The peaks at sites 2, 7, and 10 support the pattern seen by Neumman (1965). Site 2 is located near the shore of Hamilton's Parish, site 7 is in Patton's Basin, while site 10 borders Devil's Hole's cave platforms (Figures 2 and 8). Here, all three of these test-count peaks reflect both the proximity to the shore and the proximity to karst formations, which are generally higher in Mg calcite composition (Neumann, 1965). Additionally, sites 2, 7, and 10 had a mix of salinity ranges, from mesohaline to euhaline values, indicating freshwater-saltwater mixing, an important process in karst formation (Figure 4C). Overall, the decreased range of variability under decreasing test-size conditions indicates that larger test-size fractions of Quinqueloculina \& Triloculina spp. are more abundant under higher-concentrated calcitic environments. In contrast, smaller test-size fractions of Quinqueloculina \& Triloculina spp. were consistent across all sampling sites (Neumann, 1965). 


\section{$\underline{\text { Homotrema rubrum }}$}

Despite a similar trend to Quinqueloculina \& Triloculina spp. test counts, Homotrema rubrum fragment counts remain more consistent across fragment-size fractions (Figure 9). Because the analysis was performed on fragment counts rather than test counts, implications for Homotrema rubrum fragment distribution were more qualitative rather than quantitative. In theory, locations with higher fragment counts in smaller fragment-size fractions indicate highenergy environments, undergoing physical weathering processes and deposition close to the source area (Machado and Moraes, 2002). However, areas with these characteristics (sampling site 4 near Flatt's Inlet) did not yield these patterns (Figure 9). Sampling sites 2, 6, 7, and 10 are locations where fine and very fine fragment-size fractions exceeded coarse and medium fragment-size fractions. Here, the spatial distribution of these locations did not indicate any strong influence of wave energy. However, according to Walker et al. (2011), H. rubrum is most common at depths around 15 meters, which correlates well with sampling site 2 , offshore of Hamilton Sound Road (Figure 2). Meanwhile, sites 6, 7, and 10 were much deeper (18-24 meters) and located near karst platforms and basins including Green Bay caves, Patton's Basin, and Devil's Hole. Potential reasons for these findings could involve unconstrained slope gradients affecting sediment deposition and transport processes, in addition to the hydrodynamic patterns of Harrington Sound across seasons. H. rubrum must grow attached to a substrate including rock surfaces, old shells, and coral skeletons, while the other taxa are free-living on the sediment and rock surfaces. Therefore, understanding substrate availability is an additional factor in understanding why the fragments are distributed in particular patterns. Thus, when consulting previous literature on the ecological trajectories and patterns of Homotrema rubrum and the 
distribution of its fragments, this study's data do not fully agree with Walker et al. (2011) and Machado and Moraes (2002).

\section{Elphidium spp.}

The patterns seen in test-count distribution of all test-size fractions of Elphidium spp. were limited in range of variability, as count averages never exceeded 20 per site. However, there were several ecological and biological factors that explain for the trajectory peaks at sampling sites 2, 5, and 10 (Figure 10). According to Roozpeykar and Moghaddam (2015), Elphidium spp. are generally abundant in smaller test-size fractions and dominate shallow marine environments that are exposed to salinity fluctuations (brackish to marine conditions). As such, sites 2,5 , and 10 were three out of the four sampling sites with polyhaline (10-35 psu) and euhaline $(35+\mathrm{psu})$ salinity values. These results are in agreement with similar species reported in the Zagros basin, Iran (Roozpeykar and Moghaddam, 2015) (Figure 4C). Among the three sites, there were no significant geospatial implications that could be made other than salinity measurements. Thus, like Quinqueloculina \& Triloculina spp. test counts and unlike H. rubrum fragment counts, Elphidium spp. test-count trajectories agree with previous literature reports and appear to be mostly affected by salinity variability. Hence, increased test counts correspond with both high salinity and decreased test-size fractions (Figure 4C).

\section{$\underline{\text { Ammonia beccarii }}$}

Similar to the trends seen in Elphidium spp., Ammonia beccarii test-count distribution also exhibits an increase in overall count average and range of variability as test-size decreases (Figure 11). The implications behind this pattern are related to the conditional tolerance and ecological habitat of the taxon, where the increased disparity between the medium size-fraction curve and the other two test-size fractions occurs between sampling sites 6 and 10 (Figure 11). 
Here, an increased predominance of tests may indicate the presence of eutrophication, where excessive algal blooms deplete dissolved oxygen content with depth (Roozpeykar and Moghaddam, 2015) (Figure 4D and Figure 11). Interestingly, adult tests can be ecologically epiphytic, living on alga such as Corallina officinalis and Gigartina acicularis. Both of these taxa grow in temperature-constrained and salinity-fluctuating conditions (Debenay et al., 1998). Studies have found that Ammonia beccarii tests concentrate in areas with high-salinity fluctuations. However, the current study did not find peak abundances around sites with salinity flux (Figure 11). Thus, despite the fact that temperature and salinity dependence has been identified with previous studies, Ammonia beccarii test-count distribution in Harrington Sound appears to be mostly affected by dissolved oxygen. Smaller test-size fractions continued to concentrate in eutrophic conditions.

$\underline{\text { Predictive models }}$

\section{Quinqueloculina \& Triloculina spp.}

The distribution of environmental parameters in Figure 4 doesn't support a spatial correlation between high test-counts of Quinqueloculina \& Triloculina spp. and high Mg-calcitic environments as identified in Figures 2 and 8. In contrast, the quantile regression models in Figure 12 and Table 2 supported this claim. In the coarse and medium test-size fractions, both model formulas included depth and temperature, where temperature had a positive correlation with test-counts (Table 2). In general, Mg-calcite is less soluble in higher temperatures and vice versa. Thus, the relationship between $\mathrm{Mg}$-calcitic production and temperature is positive, where increased temperature is associated with increased $\mathrm{Mg}$-calcitic production. Additionally, despite sites 2, 7, and 10 being located at deeper depths (15+ meters) (Figure 4) where temperature was generally lower, the quantile models found higher test-counts of larger Quinqueloculina \& 
Triloculina spp. to correlate with higher temperature values. These findings agree with those of Neumann (1965) (Table 2). However, fine and very fine test-size fractions yielded very different model formulas and their respective inclusion-exclusions and are not in agreement with results of the larger test-size fractions. The only explanation for these discrepancies was the limited range of variability in test counts. Low variation in count data logically limits the model algorithms from calculating co-variate relationships between parameters and test-counts of fine and very fine test-size fractions.

\section{$\underline{\text { Homotrema rubrum }}$}

Even though quantile regression models for fragment-size fractions of Homotrema rubrum included most of the environmental parameters as significant contributors to fragment counts, test fragmentation requires a different interpretive approach. According to Table 3, coarse and medium fragment-size fractions included depth in the optimization process as a negative influence. Hence, decreased depth correlates with increased fragment-counts. Shallower depths generally are more affected by hydrological processes including waves and currents because lower energy is required to affect a shallow water column. In a sedimentological sense, larger fragments are less likely to be re-suspended and transported from the shallow sites compared to the smaller size classes. Hence, Machado and Moraes (2002) claimed that fragmentcounts concentrate in high-wave environments with high-energy fluctuations, and the quantile models for the larger fragment-sizes of $H$. rubrum are in agreement with their findings. In contrast, the predictive models in Figure 13 do not identify fragment-count peaks around depths of 15 meters, which is believed to be the depth preference for the taxon. This finding contrasts with Walker et al. (2011). Additionally, change in coefficient direction (positive or negative) of depth in the fine (negative influence) and very fine (positive influence) fragment-size fractions 
was most likely due to the decreased range of variability in the fragment-counts of these two fractions and decreased number of significant variables in the optimization process (Table 3).

\section{Elphidium spp.}

As a small benthic foraminiferan, Elphidium spp. are strongly influenced by increased salinities, conditions that closely resemble coastal/marine properties. Thus, predictive model assessment focused on the inclusion of salinity and its direction of influence as a controlling parameter. The inclusion-exclusion optimization in Figure 10 shows that both increased range of variability and test-count averages are seen in smaller test-size. In this model, the medium testsize fraction had the least number of parameters (only depth) found to be influential while fine and very fine test-size fractions included every variable except RDO (Table 4). Salinity was included as a positive contributor to test-counts in the fine and very fine test-size fractions. As such, increased salinity correlated with increased test-counts (Table 4). Furthermore, the coefficient direction conforms with the data reported by Roozpeykar and Moghaddam (2015), confirming the belief the observation that the abundance of small benthic forams like Elphidium spp. is largely impacted by salinity.

\section{$\underline{\text { Ammonia beccarii }}$}

Unlike the previous taxa, quantile models for test-size fractions of Ammonia beccarii were extremely limited and did not include any parameter except depth. Thus, model results disagree with both the test-count distribution in Figure 11 and those of Roozpeykar and Moghaddam (2015). Even though dissolved oxygen and temperature are both correlated with depth, the exclusion of these parameters was largely due to the small range of variability in testcount data and their linear scatter (Figure 15). Quantile regression was not able to predict testcount variability because there was minimal variability in counts when compared to the 
variability of the environmental parameters. Thus, distinct from the other taxa evaluated in this study, Ammonia beccarii test-variability was not successfully captured and modeled by quantile regression. Furthermore, these models did not agree with the plotted test-count distribution among sampling sites nor with previous literature.

$\underline{\text { Multivariate models }}$

\section{Coarse test/fragment-size interval}

Based on the npMANOVA results, important implications arise from the Subset Algorithms on test/fragment counts for the four taxa, the Subset Algorithm on environmental factor levels's contribution to test/fragment counts, and relative effect probabilities. In the coarse test/fragment-size fraction, the absence of data for Elphidium spp. and Ammonia beccarii impacted their subset results and relative effect probabilities (Table 15). This makes sense because both rotaliida taxa are known to be smaller than the other taxa.

In terms of depth, the Shallow Sandy Zone (0-10 meters) was the $1^{\text {st }}$ order statistically, distinguishable depth interval that contributed to the separation of Homotrema rubrum from the other taxa (Table 7 and Table 8). In other words, H. rubrum fragment counts were significantly different from Quinqueloculina \& Triloculina spp. test counts in the depth ranges of 0-10 m. The implications here correlate with the results of the predictive models and reports from previous literature. A high concentration of fragments correlates with shallower environments (Table 13A) that are susceptible to high-wave intensity, as reported by Machado and Moraes (2002). However, the depths were much shallower than what Walker et al. (2011) reported, where $H$. rubrum source areas concentrate around $15 \mathrm{~m}$. Correlative with the predictive models for Quinqueloculina \& Triloculina spp. test counts, it appears that the Subthermocline Zone (18-25 $\mathrm{m}$ ) was the $2^{\text {nd }}$ order statistically, distinguishable zone that contributed to the distinction between 
Quinqueloculina \& Triloculina spp., and Homotrema rubrum and the other taxa (Table 7 and Table 8). Here, the multivariate model parallels the trend in the predictive models, where the coarse test-size fraction of Quinqueloculina \& Triloculina spp. tests are concentrated in the Subthermocline Zone (Table 13A). This is true even though Quinqueloculina \& Triloculina spp. tests generally concentrate in high Mg-calcitic conditions (Neumann, 1965). In terms of temperature, the Mixed Layer $\left(26-28^{\circ} \mathrm{C}\right)$ was the $1^{\text {st }}$ order statistically, distinguishable zone that contributed to the interpretation that at least one taxon had statistically different counts (Table 7 and Table 9). Thus, both the predictive and multivariate models do not agree with the relationships identified between temperature and Quinqueloculina \& Triloculina spp. test abundances reported in Neumann (1965). However, the theoretical implications behind depth range and hydrological fragmentation of $H$. rubrum correspond with the results from both the predictive and multivariate models.

Aside from depth and temperature, statistical distinctions were made for the other parameters at the coarse test/fragment-size fraction. Here, Homotrema rubrum was $1^{\text {st }}$ order statistically, distinguishable taxon while Quinqueloculina \& Triloculina spp. was the $2^{\text {nd }}$ order statistically, distinguishable group for salinity, $\mathrm{RDO}$, and $\mathrm{pH}$. These relationships have not been documented previously. Relative effect probabilities were not appropriate to interpret and discuss due to the uneven number test/fragment counts across all four taxa.

\section{Medium test/fragment-size interval}

The trend documented in the coarse test/fragment-size fraction is also found in the medium size-fraction with regard to depth and salinity. However, due to higher test counts for Elphidium spp. and A. beccarii, more physical relationships could be evaluated against previous literature. With respect to temperature, the Subset Algorithms were able to discern statistical 
distinctions among the taxa, where Elphidium spp. was the $1^{\text {st }}$ order statistically, distinguishable taxon while Quinqueloculina \& Triloculina spp. and Homotrema rubrum were $2^{\text {nd }}$ order statistically, distinguishable taxa (Table 15). Here, the Subthermocline Layer $\left(22-24{ }^{\circ} \mathrm{C}\right)$ contributed to the statistical distinction of Elphidium spp. from the other taxa (Table 15 and Table 17). The relative effect probabilities found that Elphidium spp. tests were most likely to be abundant in temperatures of the Mixed Layer (56.5\%) (Table 21B). These agree with reports of Roozpeykar and Moghaddam (2015), if salinity and temperature possess a positive relationship in Harrington Sound. In terms of salinity, the statistical similarity between Elphidium spp. and A. beccarii could be explained by the fact that the polyhaline and euhaline salinity zones are indistinguishable (Table 15 and Table 18). In other words, Elphidium spp. and A. beccarii test counts were indistinguishable but were largely affected by increased salinity (10+ psu), which also is illustrated by their relative effect probabilities (Table 21C). Here, the medium test-size fraction of both Elphidium spp. and A. beccarii test counts were calculated to be most abundant in the Polyhaline Zone (83.3\% and $84.0 \%$ ), salinities that mimic coastal/marine settings (Table 21C). For all five parameters, the Subset Algorithms were unable to distinguish A. beccarii test counts from the other taxa, due to a limited range of variability (Table 15). However, relative effects probabilities for the medium test-size fraction of Ammonia beccarii found that abundance was concentrated in environments with higher dissolved oxygen content. This observation is inconsistent with the taxon's eutrophic preferences (Roozpeykar and Moghaddam, 2015) (Table 21D).

\section{Fine test/fragment-size interval}

Consistent with the findings for the previous test/fragment-size fractions, Homotrema rubrum and Quinqueloculina \& Triloculina spp. were the $1^{\text {st }}$ and $2^{\text {nd }}$ order statistically, 
distinguishable taxa for depth and salinity in the fine fraction size (Table 23). In terms of depth, differences in fragment counts for Homotrema rubrum were distinctive in the Shallow Sandy Zone, while differences in test counts for Quinqueloculina \& Triloculina spp. were subsequently distinctive in the Oculina Zone. Collectively, these results are consistent with data from Neumann (1965) and Machado and Moraes (2002) (Table 23 and Table 24). Here, sampling sites 2, 5, 6, and 8 were locations in the Oculina depth range (10-18 meters) (Figure 2 and Figure 12C), as well as collection sites close to karst in Patton's Basin, Green Bay caves, South Basin, and Hamilton's Parish caves (Figure 2 and Figure 3). With preference to intermediate depths (Table 29A) and proximity to karst platforms, the multivariate model for the fine test-size fraction of Quinqueloculina \& Triloculina spp. agrees with Neumann (1965). Because shallower depths appeared to influence $H$. rubrum fragment counts more than at other depth zones (Table 29A), the multivariate model for the fine fragment-size fraction of this taxon agreed with reports by Machado and Moraes (2002).

Unlike previous npMANOVA Subset Algorithms, Subset Algorithms for salinity distinguished three out of the four taxa as statistically distinctive from one another in the fine test/fragment-size fraction (Table 23). As small benthic foraminifera, Elphidium spp. and A. beccarii were expected to be influenced by salinity. However, only A. beccarii appears to be influenced by salinity at this fraction size, specifically by the statistical congruence between the mesohaline and euhaline zones (Figure 23 and Table 26). This was quite unusual when only considering the Subset Algorithms, but the relative effect probabilities provided numerical insight that ultimately agreed with data from Roozpeykar and Moghaddam (2015). Here, the fine test-size fraction of Elphidium spp. and Ammonia beccarii was most abundant in polyhaline and euhaline conditions (Table 29C). Thus, in models where Subset Algorithm results did not 
completely agree with relative effect probabilities, this situation was most likely due to the limitations of the iterative optimization of Subset Algorithms, in which relative effects probabilities serve as the ad-hoc compliment.

\section{Very fine test/fragment-size interval}

Depth and salinity were the only environmental parameters that yielded statistically, distinguishable taxa in the very fine test/fragment-size fraction of the Subset Algorithms on test/fragment counts. Here, Elphidium spp. and Ammonia beccarii were the $1^{\text {st }}$ and $2^{\text {nd }}$ order statistically distinct taxa (Table 31). The parameters that weren't significant, or were not able to statistically distinguish taxa at the very fine test/fragment-size, were, in part, subjected to a limited range of variability in counts. Both rotaliids agreed with the data provided by

Roozpeykar and Moghaddam (2015), regarding shallow depth preferences and epiphytic ecology of these taxa with coral species. As the $1^{\text {st }}$ order statistically, distinguishable taxon, Elphidium spp. test counts were significantly affected by the Shallow Sandy Zone (Table 32), confirming ecological patterns surrounding shallow marine environments. As the $2^{\text {nd }}$ order statistically, distinguishable taxon, $A$. beccarii test counts were significantly affected by the Oculina Zone (Table 32), the depth range in which coral species are most abundant. In contrast, the relative effects probabilities identified Elphidium spp. as most abundant in the Oculina Zone and Subthermocline Zone, while Ammonia beccarii were most abundant in the Shallow Sandy Zone (Table 37). In terms of salinity, Homotrema rubrum and Quinqueloculina \& Triloculina spp. were the $1^{\text {st }}$ and $2^{\text {nd }}$ order statistically, distinguishable taxa, where Homotrema rubrum was most affected by mesohaline salinity values and Quinqueloculina \& Triloculina spp. was most affected by polyhaline salinity values (Table 34). The relative effect probabilities identified that the very fine size-fraction of Homotrema rubrum fragments was most abundant in euhaline and 
polyhaline zones. In contrast, the very fine size-fraction of Quinqueloculina \& Triloculina spp. tests was most abundant in polyhaline conditions, exhibiting some disagreement with the Subset Algorithms results (Table 37B).

\section{$\underline{\text { Ordination }}$}

The only ordinal model that illustrated significant visualization and statistical significant results among the taxa was the ordinal plot produced by nMDS using the Bray Distance scale metric (Figure 17). All test/fragment-size fractions for Quinqueloculina \& Triloculina spp., $H$. rubrum, and Elphidium spp. were found to be dissimilar, where each size-fraction of each taxon was spatially clustered around other fraction sizes of its respectable taxon. However, the distribution of $A$. beccarii in the nMDS plot shows some inconsistencies. The medium test-size fraction plots closer to test-size fractions of Elphidium spp. than the other two test-size fractions of Ammonia beccarii (Figure 17). A possible explanation for this trend could be explained by the patterns seen in the multivariate models at the medium test/fragment-size fraction (Table 15), where the Subset Algorithms for test/fragment-counts found Elphidium spp. and Ammonia beccarii statistically similar to one another for three out of the five parameters. Meanwhile, the Subset Algorithms for counts for the fine and very fine fraction sizes were more statistically distinct (Table 15, Table 23, and Table 31). The iterative patterns seen in both npMANOVA and nMDS could be explained by some inconsistent variability in the original dataset, where the medium test-size fraction of $A$. beccarii had an unusual count spread.

\section{$\underline{\text { Geospatial models }}$}

\section{Environmental parameters}

Despite the differences between the triogram smoothing method in AQR (Koenker, 2018) and the triangular interpolation process in TIN, the spatial trajectories were similar-to-identical 
in both models. This indicates that non-parametric distinctions were prevalent in profile analysis, while they were negligible in geospatial analysis. Thus, both AQR and TIN can be considered viable options for research studies requiring geospatial analysis. TIN has the primary spatialization tool in recent years. Nevertheless, TIN was more compatible for overlaying contour boundaries for test/fragment-counts.

\section{Quinqueloculina \& Triloculina spp.}

In all four test-size fractions of Quinqueloculina \& Triloculina spp. (Figures 21-24), test counts increased as water depth increased, temperature decreased, and RDO decreased. Test counts also increased in areas near karst formations (e.g., Devil's Hole, Green Bay caves, Hamilton's Parish caves). This trend also was seen across predictive and multivariate models, where test concentration in lower temperature zones was in contrast with Neumann (1965). This observation applies to miliolid foraminifera's abundance preferences toward high Mg-calcitic environments. Furthermore, the decrease in range of test-count variability across profile and geospatial analyses affirmed model consistencies based on the original datasets. Thus, spatial maps of all test-size fractions of Quinqueloculina \& Triloculina spp. illustrated the trends, patterns, and trajectories seen across the different model results, where Quinqueloculina \& Triloculina spp., irrespective of test-size fractions, concentrated in deeper and colder regions in Harrington Sound.

\section{Homotrema rubrum}

Fragmentation results are interpreted differently than test counts. Thus, for spatial correlations, fragment concentration was assessed based on proximity to shallower depths and tidal channels. In all four fragment-size fractions of H. rubrum (Figures 25-28), fragment counts increased towards areas with shallower depths, higher temperatures, lower salinity, higher 
dissolved oxygen, and higher $\mathrm{pH}$. When compare to data of Machado and Moraes (2002), the TINs for all fragment-size fractions of H. rubrum are concentrated in shallower depths. In contrast, the results from TINs did not agree with the data from Walker et al. (2011), who claimed that $H$. rubrum is generally abundant around depths of 15 meters. Sampling sites 2 and 5 were the only sites in that depth range, and ultimately did not have an abundance of fragment counts (Figure 9). Thus, spatial maps of all fragment-size fractions illustrated the trends, patterns, and trajectories seen across the different model results. $H$. rubrum, regardless of fragment-size fractions, concentrated in shallower locations in Harrington Sound.

\section{Elphidium spp.}

The analytical approach for test-counts of Elphidium spp. was consistent with previous modeling technique. An assessment of the TINs focused on the spatial relationships between depth, salinity, and test counts to determine if the data of the current study are consistent with those of Roozpeykar and Moghaddam (2015). The medium and fine test-size fractions yielded contour trajectories that do not correlate with depth and salinity, while the very fine test-size fraction correspond with increased depth and salinity (Figures 29-31). The fact that there is a limited correlation to depth and salinity in the larger test-size fractions is largely due to the limited range of variability and overall test-count averages for this taxon, both of which would create difficulty to interpolate spatial relationships (Figure 10). The very fine test-size fraction displayed a strong correlation with depth and salinity due to the increased range of variability and test-count averages (Figure 10). Furthermore, test-counts are concentrated in high salinity zones, which resemble coastal/marine conditions, agreeing with conclusions of Roozpeykar and Moghaddam (2015). Thus, unlike Quinqueloculina \& Triloculina spp. and H. rubrum, only results of the smallest test-size fraction were in agreement with previous literature. 


\section{$\underline{\text { Ammonia beccarii }}$}

Roozpeykar and Moghaddam (2015) and Debenay et al. (1998) identified depth and RDO as the physical factors influencing Ammonia beccarii test counts. Yet, none of the test-size fractions of this taxon had similar contour trajectories/boundaries (Figures 32-34), which also were captured in the predictive models (Table 5). Similar to Elphidium spp., the inconsistent correlations with parameters in test-size fractions of Ammonia beccarii were largely due to the differing range of variability and overall test-count averages for this taxon. In the medium fraction size, high test-counts did not correlate with depth but correlated with increased RDO. This relationship is unusual due to the taxon's eutrophic preferences (Roozpeykar and Moghaddam, 2015). In the fine fraction size, high test-counts are concentrated in deeper depth ranges and lower RDO, affirming the preference for eutrophic conditions and depth ranges most suitable for coral species on which Ammonia beccarii live (Debenay et al., 1998). The very fine fraction size correlated with both depth and RDO, where high test-counts correspond with shallower depths but higher RDO. This finding is similar to the medium fraction size and, therefore, in contrast with Roozpeykar and Moghaddam (2015). Furthermore, similar to the small benthic foram Elphidium spp., Ammonia beccarii is also affected by salinity. But, the very fine test-size fraction was found to correlate with lower salinity values, ultimately a finding that is inconsistent with previous literature.

\section{$\underline{\text { Model limitations and prospects }}$}

Developing and determining the most effective quantitative modeling approach is a theoretical process based on the frequent non-normality of scientific data. However, modeling limitations come with every technique. The predictive models utilized quantile regression, which estimates the conditional median of the data, to model and predict response. In theory, this is an 
excellent approach to constrain for non-normality, but optimal effectivity and efficiency requires large numbers of observations and bigger datasets. Therefore, conducting predictive modeling on 18 trials, athough still very effective, resulted in weaker predictive confidence. For the purpose of this study, these models served to identify significant physical-chemical parameters and their direction of influence. Thus, it serves as the first phase of the modeling process.

The multivariate models incorporating MANOVA under a rigorous randomization process was used to determine what factor level of a specific parameter contributed to highest test/fragment abundance. In theory, this was the perfect modeling technique when considering the co-existence of all four taxa in a predictive procedure. However, the model's biggest limitation was the singular explanatory variable constraint. Four taxa only can be modeled against one environmental at a time, which altered the npMANOVA analysis due to the nonparametric assumptions of the dataset. Thus, as a second step in the modeling, npMANOVA, complimented the quantile regression protocol by specifying variable zonation and its relationship to test/fragment count abundances.

The geospatial models utilized AQR and TIN functions to draw spatial maps of environmental parameters and contour boundaries for test/fragment counts, which served to provide the three-dimensional aspect of the modeling phases. That is, the previous two modeling phases focused on changes in a depth profile but neglected the spatial and temporal relationships in Harrington Sound. Thus, in theory and in many applications, spatial mapping is an incredibly powerful and useful tool to provide a new dimension to statistical modeling. The model's biggest drawbacks were the lack of sampling points and measurements throughout Harrington Sound. Due to our inability to collect samples in the North Basin, the spatial maps only analyzed and interpreted approximately $50 \%$ of the overall area of Harrington Sound. Thus, as a common 
problem seen in many research studies, obtaining sufficient data was a limitation for modeling procedures. Moving forward, power analyses could also be used to estimate required sampling efforts to improve the sensitivity of the models.

This study's methodology and results proved to be extremely effective in predicting ecological distribution of test-size concentrations in a complex system, despite the study's limitations. Thus, as one of the very few studies utilizing quantile regression and npMANOVA to create predictive, multivariate, and geospatial models, this study urges the increased usage of these techniques in future research focused on similar interests and inquiries. 


\section{REFERENCES}

Bates, N., 2017: Twenty Years of Marine Carbon Cycle Observations at Devils Hole Bermuda Provide Insights into Seasonal Hypoxia, Coral Reef Calcification, and Ocean Acidification. Frontiers in Marine Science, v.4, no. 36.

Blackmon P.D., and R. Todd, 1959: Mineralogy of some foraminifera as related to their classification and ecology. Journal of Paleontology, v. 33, p. 1-15.

Boudagher-Fadel, M.K., 2015: Biostratigraphic and Geological Significance of Planktonic Foraminifera. 2, Revised ed., UCL Press.

Burchett, W.W., A. R. Ellis, S.W. Harrar, and A. C. Bathke, 2017: Nonparametric Inference for Multivariate Data: The R Package npmv. Journal of Statistical Software, v. 76, no. 4, p. $1-18$.

Cade, B.S., and B.R. Noon, 2003: A Gentle Introduction to Quantile Regression for Ecologists. Frontiers in Ecology and the Environment, v. 1, no. 8, p. 412-420.

Clarke, K. R., 1993: Non-parametric multivariate analysis of changes in community structure. Australian Journal of Ecology, no.18, 117-143.

de Vernal, A., and H. M. Bilodeau, 1996: Techniques de préparation et d'analyse en micropaléontologie. Les cahiers du GEOTOP, no. 3, p. 16-27.

Debenay, J.-P., Bénéteau, E., Zhang, J., Stouff, V., Geslin, E., Redois, F., \& M. FernandezGonzalez, 1998: Ammonia beccarii and Ammonia tepida (Foraminifera): morphofunctional arguments for their distinction. Marine Micropaleontology, v. 34, p. 235-244.

Erlenkeuser, H., R. Dawson, D. Futterer, H. Heinrich, G. Leibezeit, D. Meischner, P. Muller, and G. Wefer, 1981: Environmental changes during the last 9000 years as reflected in a sediment core from Harrington Sound, Bermuda. The Harrington Sound Project, Kiel University, Bermuda Biological Station for Research, Special Publication No. 19, p. 2360.

Fairfield, N., G., Kantor, D., Jonak, and D. Wettergreen, 2009: Autonomous exploration and mapping of flooded sinkholes, The International Journal of Robotics Research, v. 29, p. 748.

Friedman, G.M., 1964: Early diagenesis and lithification in carbonate sediments. Journal of Sedimentary Petrology, v. 34, p. 777-813.

Hansen, M., Kooperberg, C., and S. Sardy, 1998: Triogram Models: Journal of the American 
Statistical Association, v. 93, p. 101-119.

Javaux, E. J., and D. B. Scott, 2003: Illustration of Modern Benthic Foraminifera from Bermuda and Remarks on Distribution in Other Subtropical/tropical Areas. Palaeontologia Electronica v. 6, no. 4.

Koenker, R., 2018: Quantile regression in R: A vignette. https://cran.rproject.org/web/packages/quantreg/vignettes/rq.pdf

Koenker, R., and K.F. Hallock, 2001, Quantile Regression: Journal of Economic Perspectives, v. 15 , p. $143-156$.

Koenker, R., and I. Mizera, 2004: Penalized Triograms: Total Variation Regularization for Bivariate Smoothing. Journal of the Royal Statistical Society. Series B (Statistical Methodology), v. 66, no. 1, pp. 145-163.

Legendre, P., and L. Legendre, 1998: Numerical Ecology. Elsevier Science, Amsterdam, v. 24.

Loeblich, A.R., and H. Tappan, 1964: Treatise on Invertebrate Paleontology, Part C: Protista 2, Sarcodina, chiefly "Thecamoebians" and Foraminiferida. Geological Society of America and University of Kanzas Press, Lawrence.

Lutze, G.F., and G. Wefer, 1980, Habitat and asexual reproduction of Cyclorbicula compressa (Orbigny), Soritidae: The Journal of Foraminiferal Research, v. 10, p. 251-260.

Machado, A. J., and S. S. Moraes, 2002: A note on the occurrence of the encrusting foraminifera Homotrema rubrum in reef sediments from two distinctive hydrodynamic settings. Anais da Academia Brasileira de Ciências, v. 74, no. 4, p. 727-735.

Morris, B., Barnes, J., Brown, F., and J. Markham, 1977: The Bermuda Marine Environment, A report of the Bermuda inshore waters investigations, Bermuda Biological Station, Bermuda.

Neumann, A.C., 1965: Processes of Recent Carbonate Sedimentation in Harrington Sound, Bermuda, Bulletin of Marine Science, v. 15, p. 987-1035.

Oksanen, J., 2015: Multivariate Analysis of Ecological Communities in R: vegan tutorial. $R$ Package Version 1.

Parsons, R.J., Nelson, C.E., Carlson, C.A., Denman, C.C., Andersson, A.J., Kledzik, A.L., Vergin, K.L., Mcnally, S.P., Treusch, A.H., and S.J. Giovannoni, 2014: Marine bacterioplankton community turnover within seasonally hypoxic waters of a subtropical sound: Devils Hole, Bermuda: Environmental Microbiology, v. 17, p. 3481-3499. 
Pohlman, J.W., 2011: The biochemistry of anchialine caves: progress and possibilities, Hydrobiologia, v. 677, no.1, p. 33-51.

Roozpeykar, A., and I.M., Moghaddam, 2015: Benthic foraminifera as biostratigraphical and paleoecological indicators: An example from Oligo-Miocene deposits in the SW of Zagros basin, Iran. Geoscience Frontiers, v. 7, no. 1, p. 125-140.

Schweizer, M., Polovodova, I., Nikulina, A., and J. Schönfeld, 2011: Molecular identification of Ammonia and Elphidium species (Foraminifera, Rotaliida) from the Kiel Fjord (SW Baltic Sea) with rDNA sequences. Helgoland Marine Research, v. 65, p. 1-10.

Shapiro, S. S., and M. B. Wilk, 1965: “An Analysis of Variance Test for Normality (Complete Samples).” Biometrika, vol. 52, no. 3/4, p. 591-611.

Stoffer, J.L., and T.M. Iliffe, 2013: A Hydrological Model of Harrington Sound, Bermuda and its Surrounding Cave Systems [M.S. Thesis]: Texas A\&M University, 109 p.

Taylor, P.D. and M.A. Wilson, 2003: Palaeoecology and evolution of marine hard substrate communities: Earth Sci. Rev., v. 62, p. 1-103.

Vacher, H.L. and T.M. Quinn, 2004: Geology and hydrogeology of carbonate islands, first ed. Elsevier Science, Amsterdam.

Walker, S. E., Parsons-Hubbard, K., Powell, E.N., and C.E. Brett, 2011: Alpha and beta diversity of encrusting foraminifera that recruit to long-term experiments along a carbonate platform-to-slope gradient: Paleoecological and paleoenvironmental implications. Paleoecology, v. 312, no. 3-4, p. 325-349.

Wefer, G., Killingley, J.S., and G.F. Lutze, 1981, Stable isotopes in recent larger foraminifera: Palaeogeography, Palaeoclimatology, Palaeoecology, v. 33, p. 253-270.

Wefer, G., and W.H. Berger, 1980, Stable Isotopes in Benthic Foraminifera: Seasonal Variation in Large Tropical Species: Science, v. 209, p. 803-805.

Warton, D.I., Wright, T.W., and Y. Wang, 2012: Distance-based multivariate analyses confound location and dispersion effects. Methods in Ecology and Evolution, v. 3, p. 89-101. 


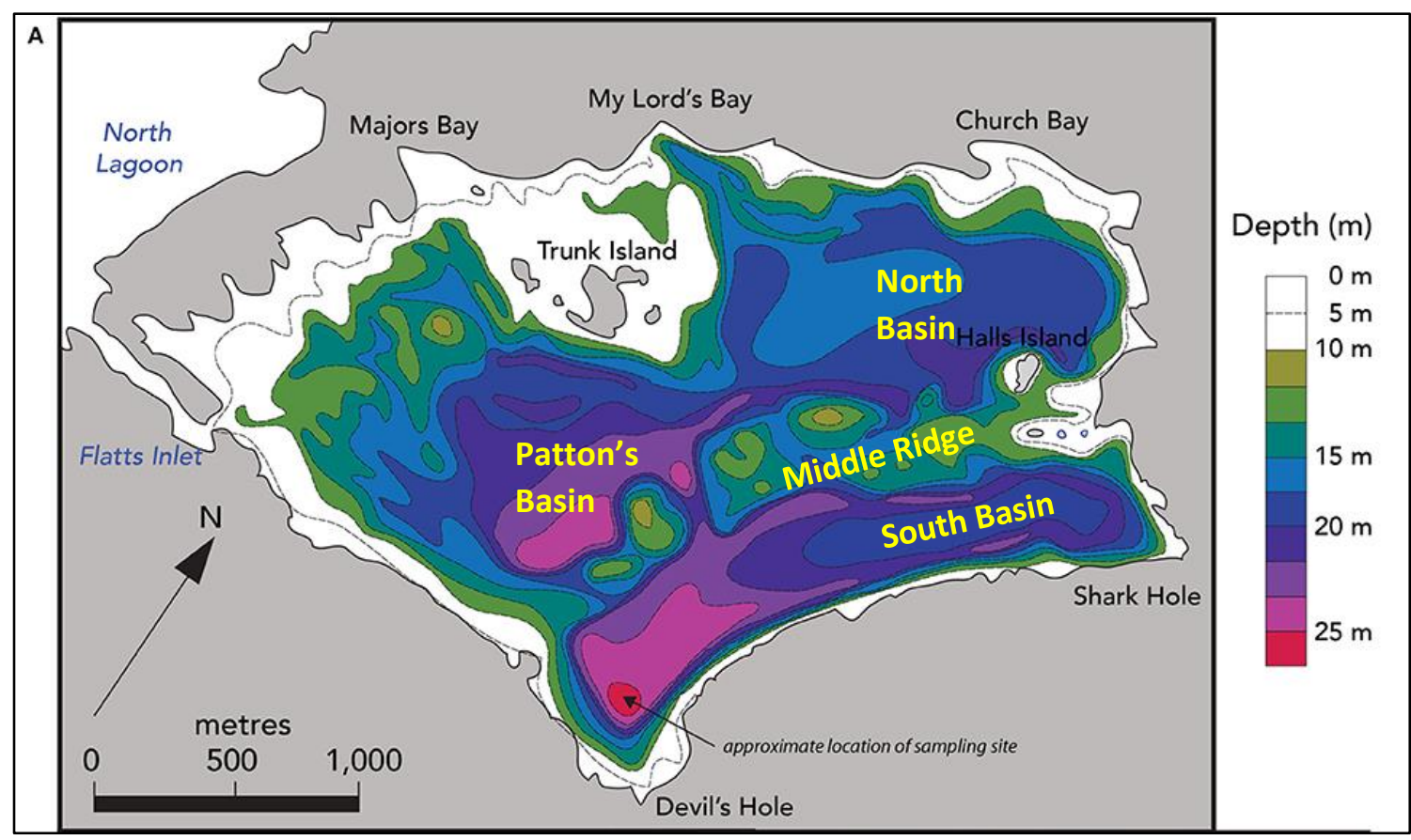

Figure 1. Depth Map of Harrington Sound with geologic features labeled (yellow) after Bates, 2017. 


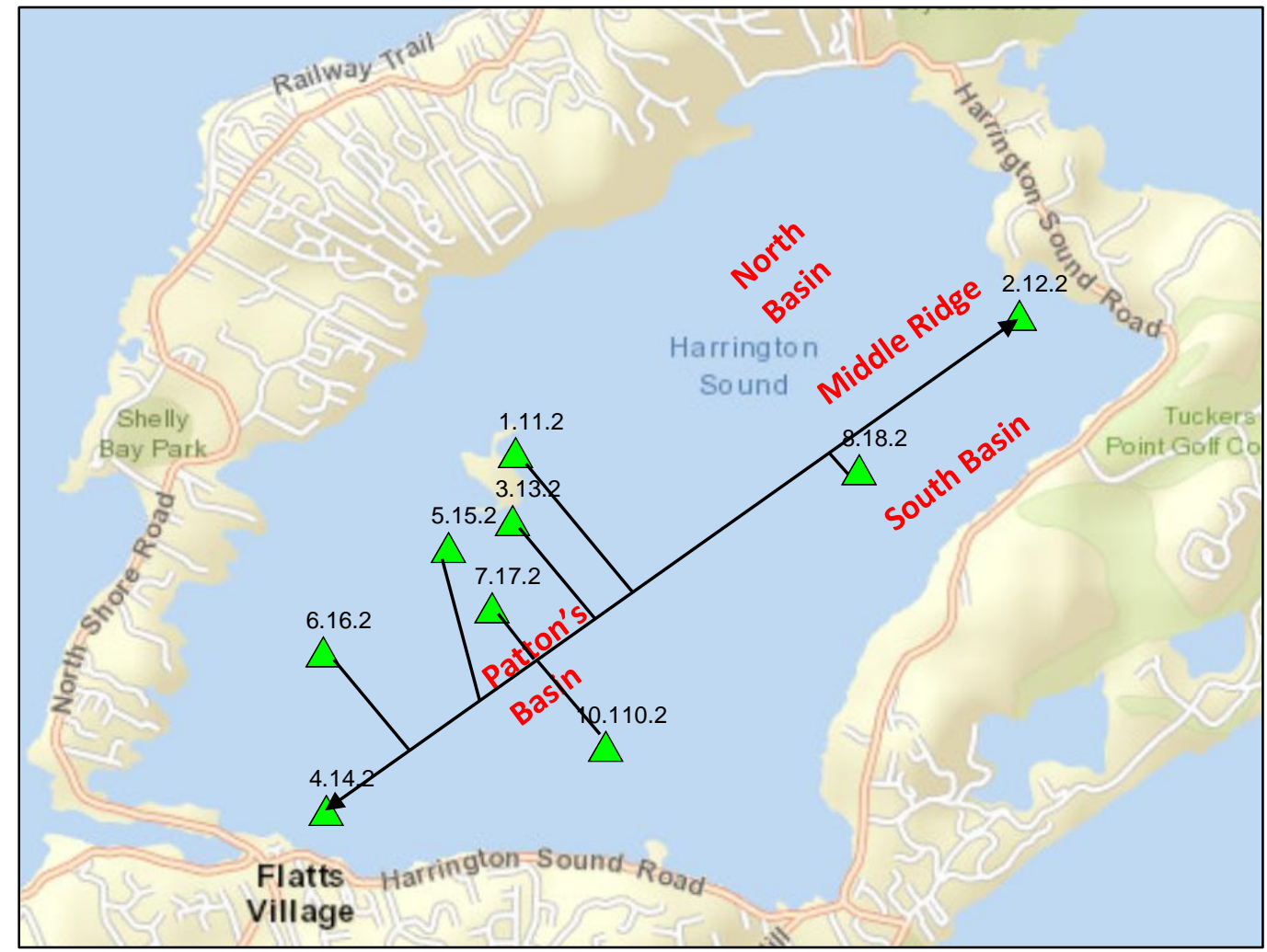

Figure 2. Sediment Sample Sites with geologic features labeled (red) and transect sketched (black). 


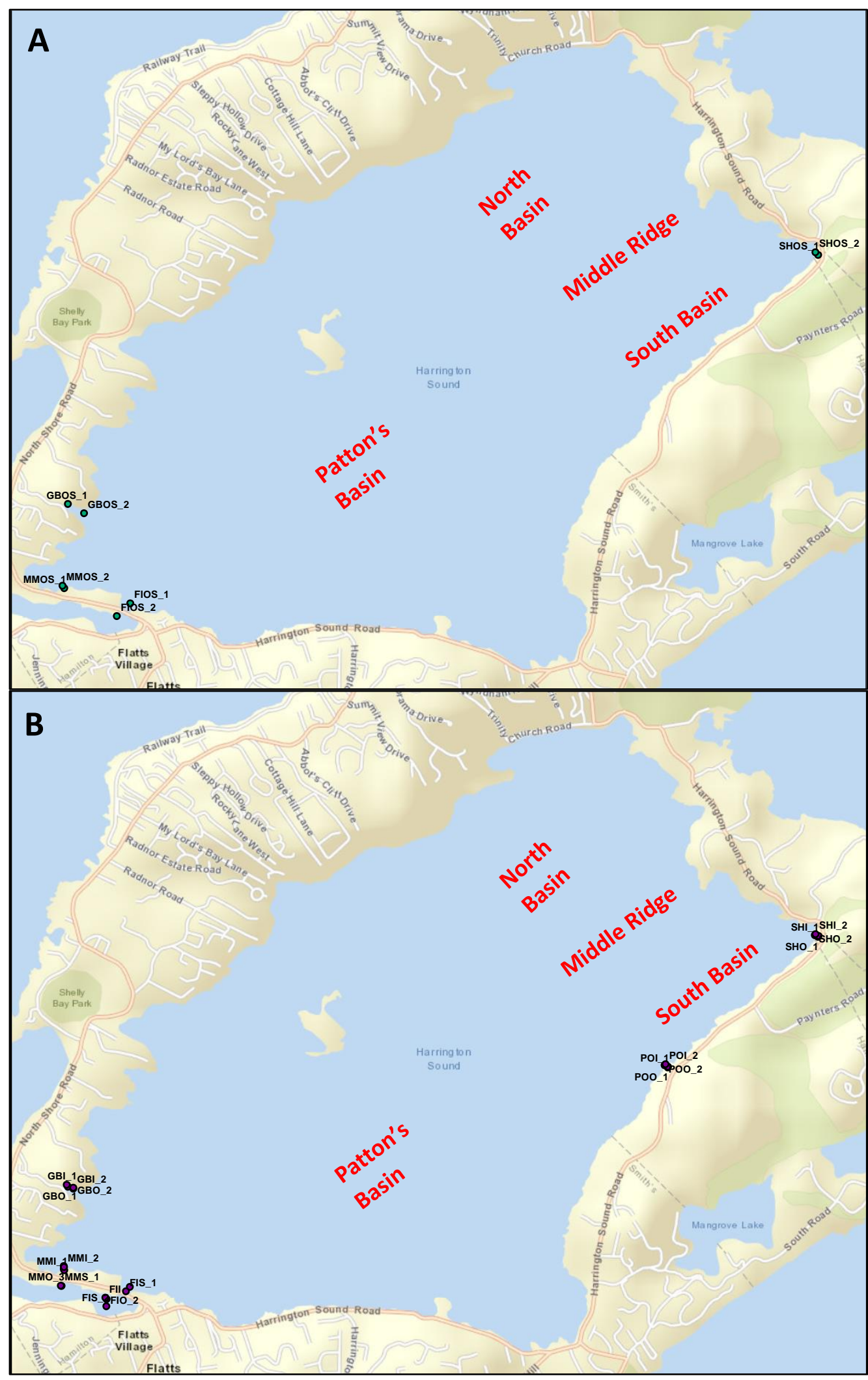

Figure 3. Water Chemistry

Sampling Sites in

Harrington

Sound. (A)

Summer samples

from July 1, 2018.

(B) Winter

samples from

January 18, 2019.

GBO - Green Bay

Caves during

outgoing tide;

GBI - Green Bay

caves during

incoming tide;

MMO -Manmade

Cave during

outgoing tide;

MMI - Manmade

Cave during

incoming tide;

MMS - Manmade

Cave during slack

tide; FIO - Flatt's

Inlet during

outgoing tide; FII

- Flatt's Inlet

during incoming

tide; FIS - Flatt's

Inlet during slack

tide; SHO - Shark

Hole during

outgoing tide; SHI

- Shark Hole

during incoming

tide; POO - Post

Office Cave

during outgoing

tide; POI - Post

Office Cave

during incoming

tide. 
A

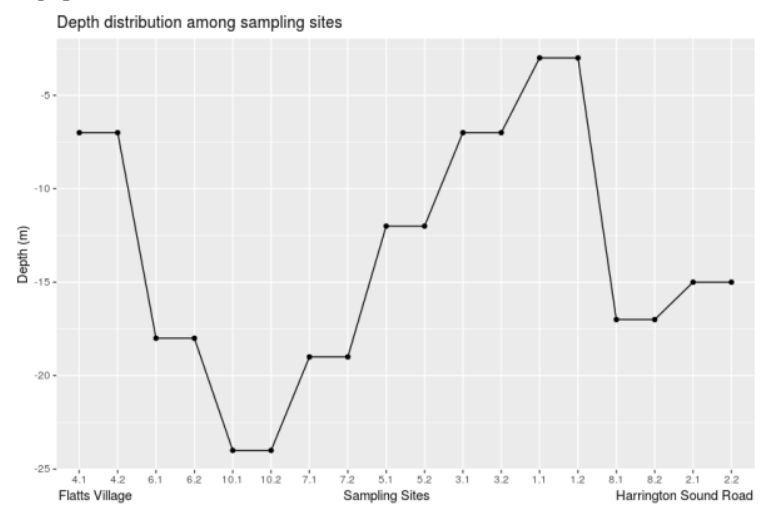

C

Salinity distribution among sampling sites

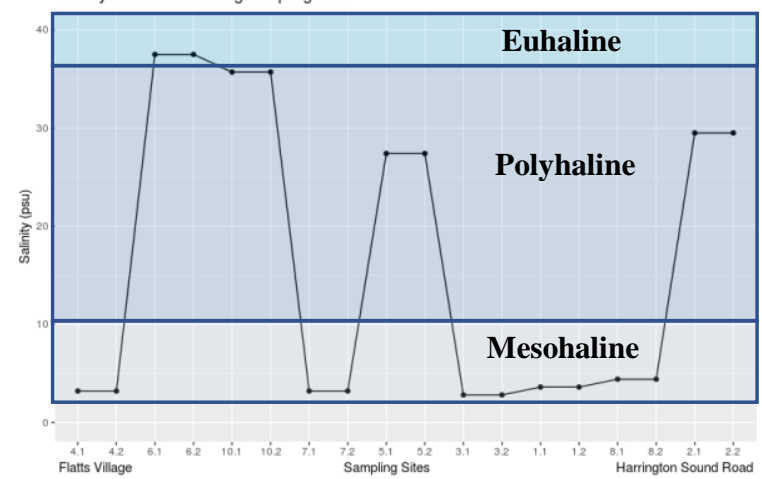

E

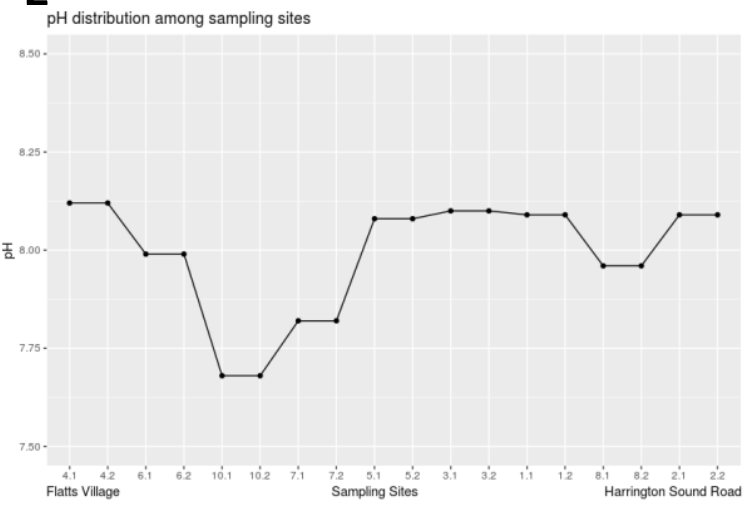

B

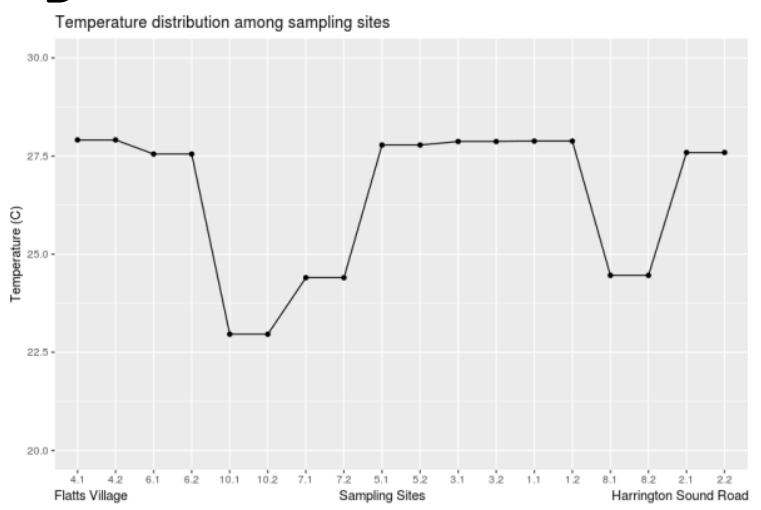

D

RDO distribution among sampling sites

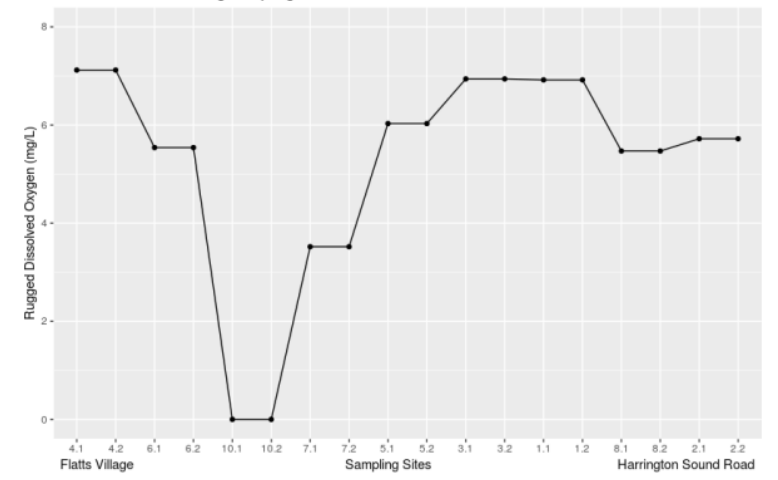

Figure 4. Physical and geochemical distribution under each sampling location. (A) Depth (m); (B) Temperature (C); (C) Salinity (psu); (D) RDO (mg/L); (E) pH. 
A

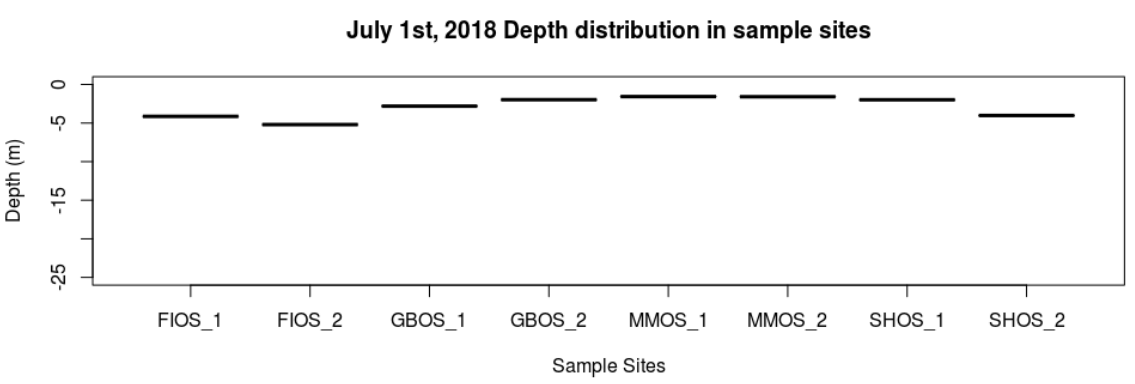

B

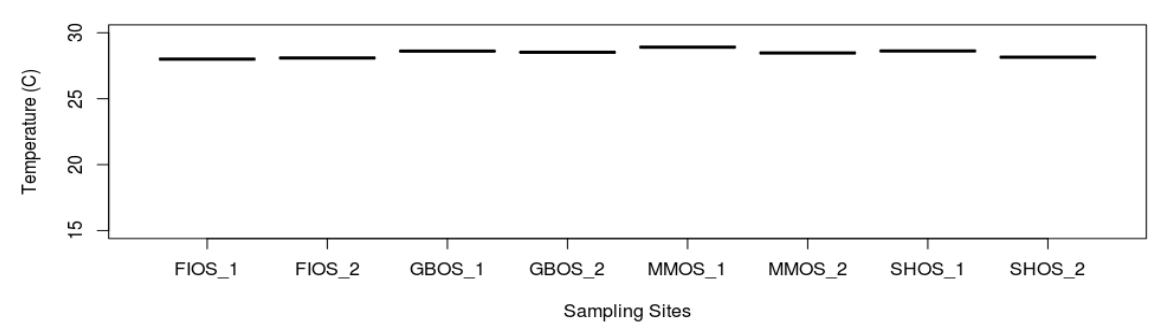

C

July 1st, 2018 Salinity distribution in sampling sites

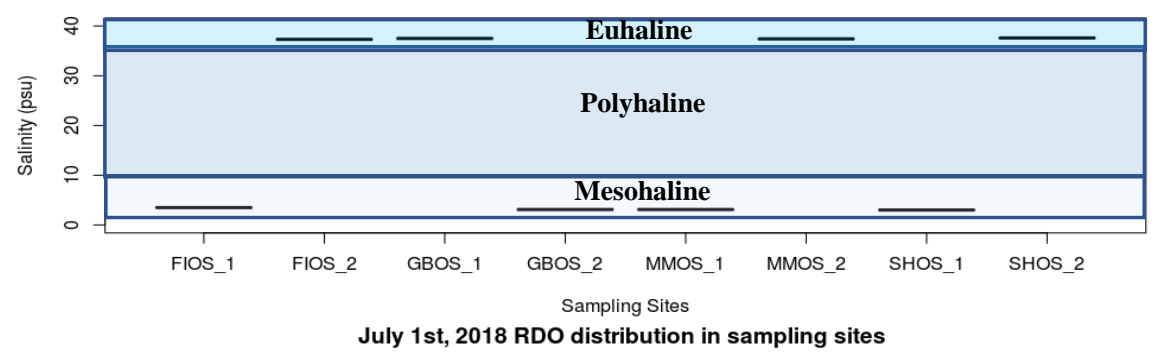

D

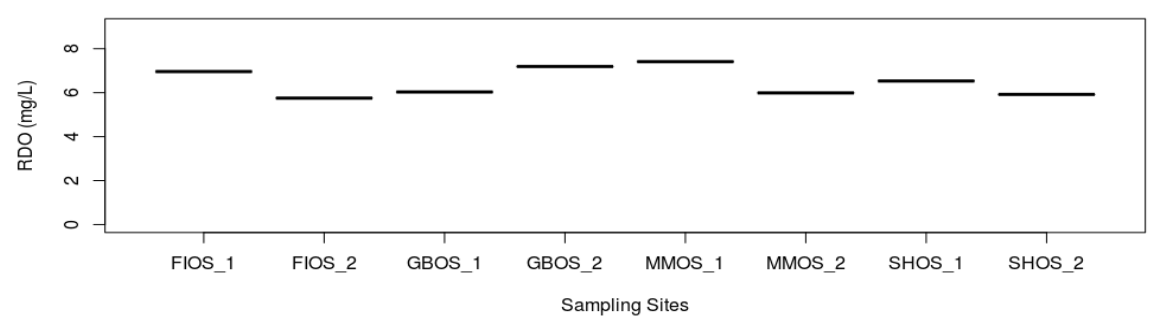

E

July 1st, 2018 pH distribution in sampling sites

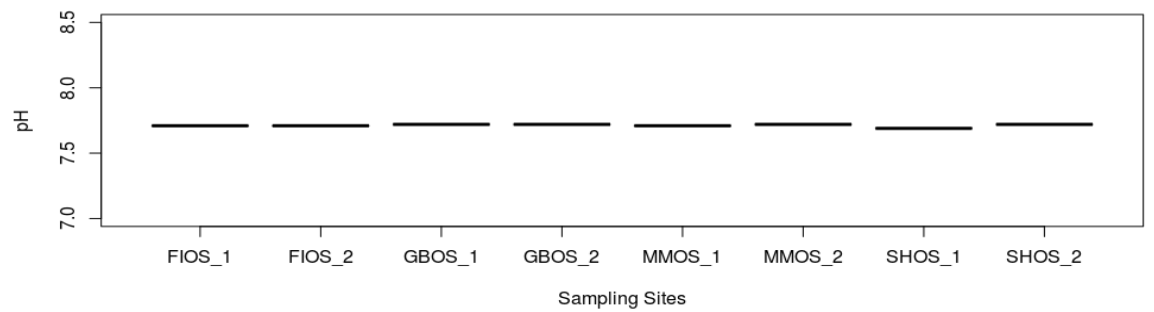

Figure 5. Physical and geochemical distribution of cave systems and Flatt's Inlet for July $\mathbf{1}^{\text {st }}, 2018$ under each sampling location. (A) Depth (m); (B) Temperature (C); (C) Salinity (psu); (D) RDO (mg/L); (E) pH. GBO Green Bay Caves during outgoing tide; MMO - Manmade Cave during outgoing tide; FIO - Flatt's Inlet during outgoing tide; SHO - Shark Hole during outgoing tide. 
A

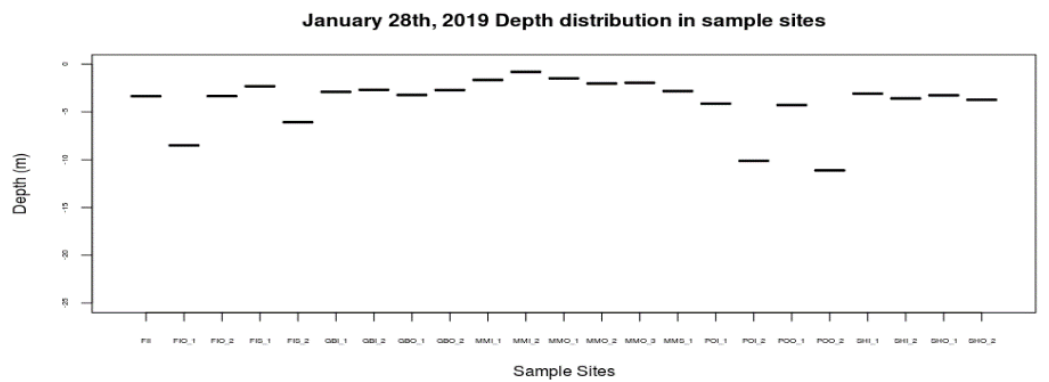

B

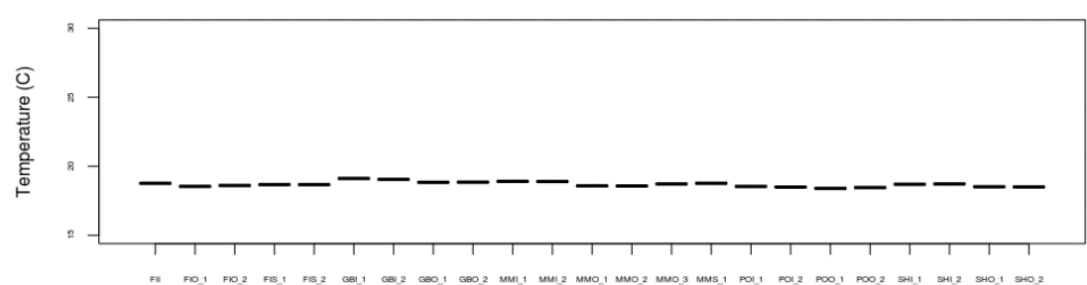

Sampling Sites

C

January 18th, 2019 Salinity distribution in sampling sites

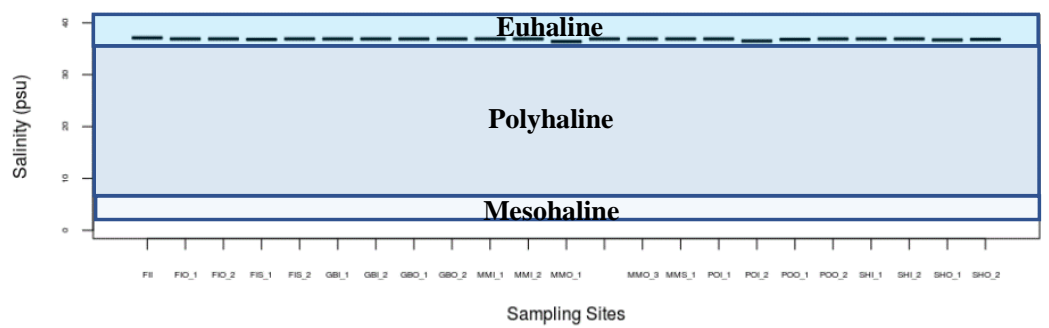

D

January 18th, 2019 RDO distribution in sampling sites

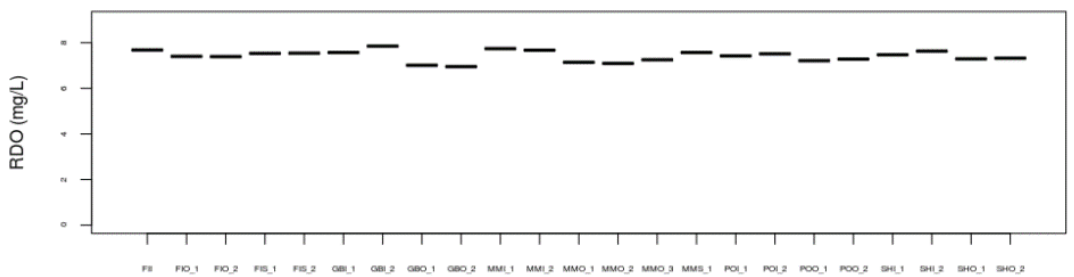

Sampling Sites

E

January 18 th, $2019 \mathrm{pH}$ distribution in sampling sites

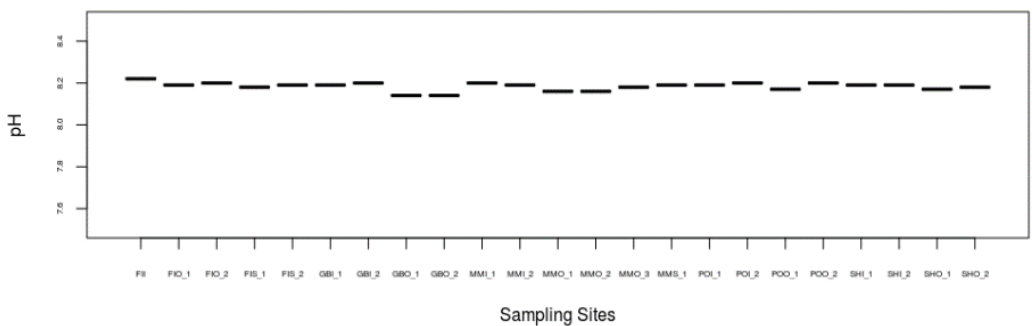

Figure 6. Physical and geochemical distribution of cave systems and Flatt's Inlet for January $18^{\text {th }}, 2019$ under each sampling location. (A) Depth (m); (B) Temperature (Celsius); (C) Salinity (psu); (D) RDO (mg/L); (E) pH. GBO - Green Bay Caves during outgoing tide; GBI - Green Bay caves during incoming tide; MMO - Manmade Cave during outgoing tide; MMI - Manmade Cave during incoming tide; MMS - Manmade Cave during slack tide; FIO - Flatt's Inlet during outgoing tide; FII - Flatt's Inlet during incoming tide; FIS - Flatt's Inlet during slack tide; SHO - Shark Hole during outgoing tide; SHI - Shark Hole during incoming tide; POO - Post Office Cave during outgoing tide; POI - Post Office Cave during incoming tide. 
A

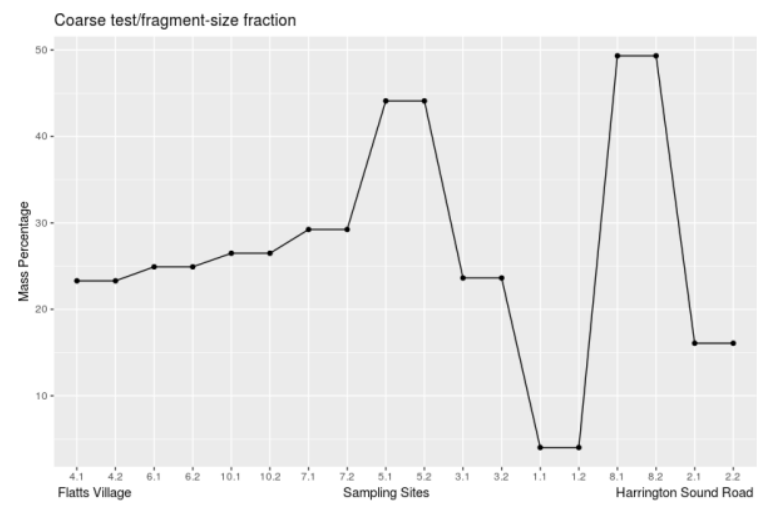

C

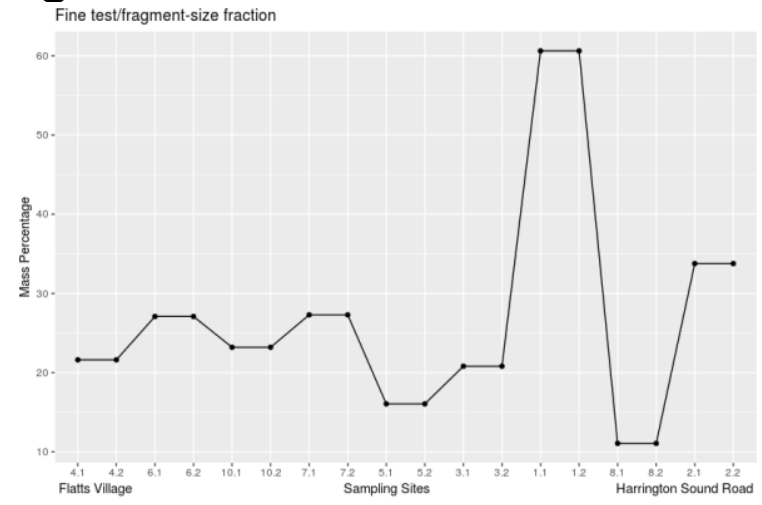

B

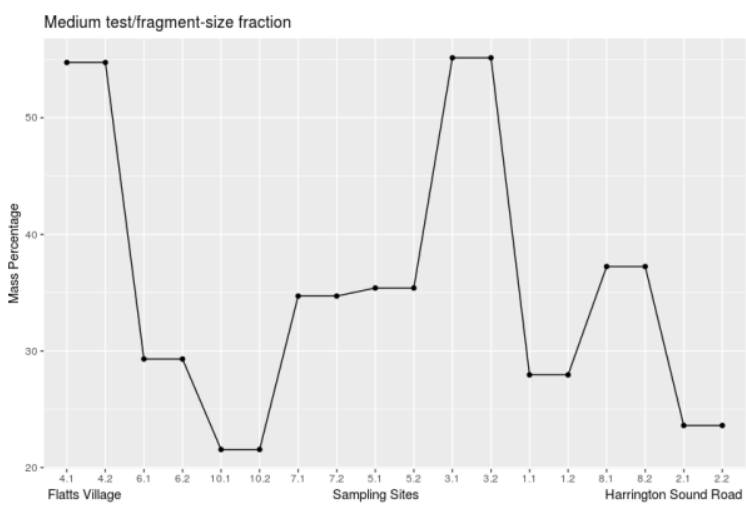

D

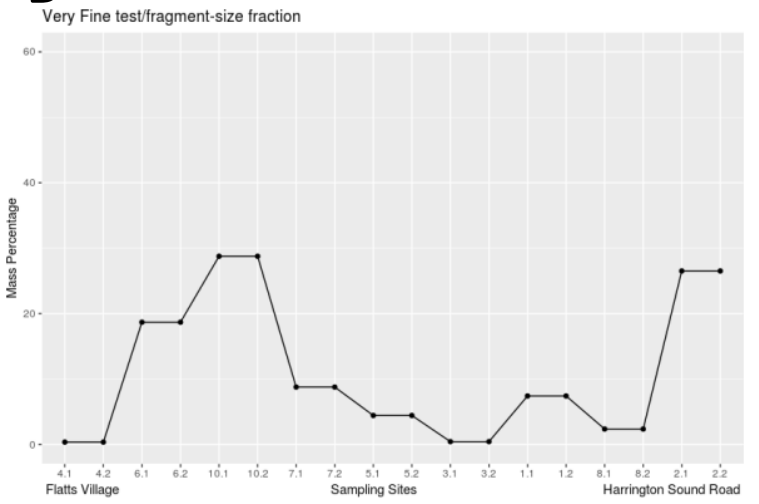

Figure 7. Mass distribution in each test/fragment-size interval under each sampling location. (A) Coarse test/fragment-size interval; (B) Medium test/fragment-size interval; (C) Fine test/fragment-size interval; (D) Very fine test/fragment-size interval. 
Quinqueloculina \& Triloculina spp. distribution among sample sites

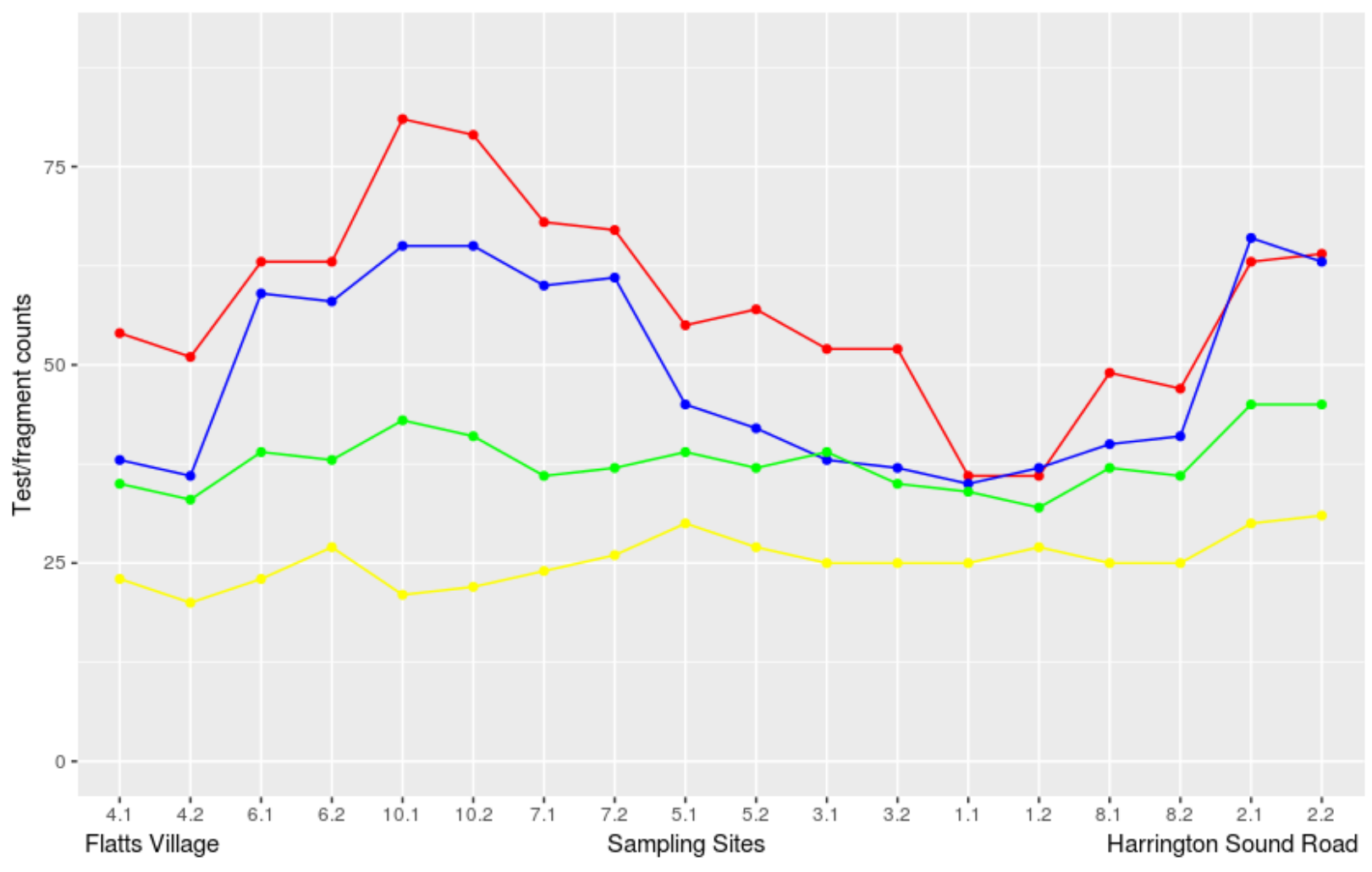

Legend

---- Coarse $(1 / 2+\mathrm{mm})$---- Medium $(1 / 4-1 / 2 \mathrm{~mm}) \quad$---- Fine $(1 / 8-1 / 4 \mathrm{~mm}) \quad$-- Very fine $(1 / 16-1 / 8 \mathrm{~mm})$

Figure 8. Test count distributions of Quinqueloculina \& Triloculina spp. at each sampling location in different test-size intervals (Coarse, Medium, Fine, Very fine). 
Homotrema rubrum fragment distribution among sample sites

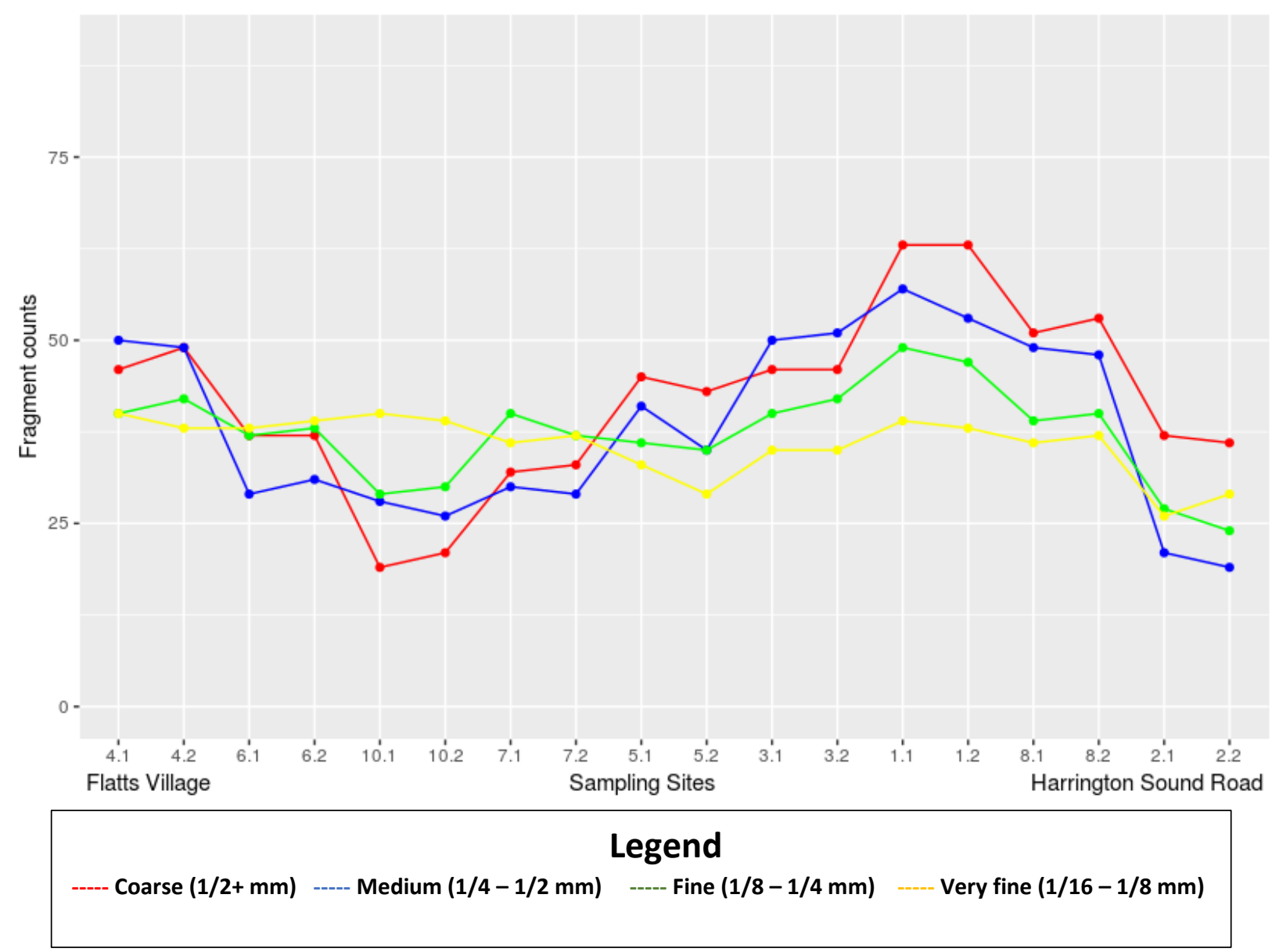

Figure 9. Fragment count distributions of Homotrema rubrum at each sampling location in different fragment-size intervals (Coarse, Medium, Fine, Very fine). 
Elphidium spp. distribution among sample sites

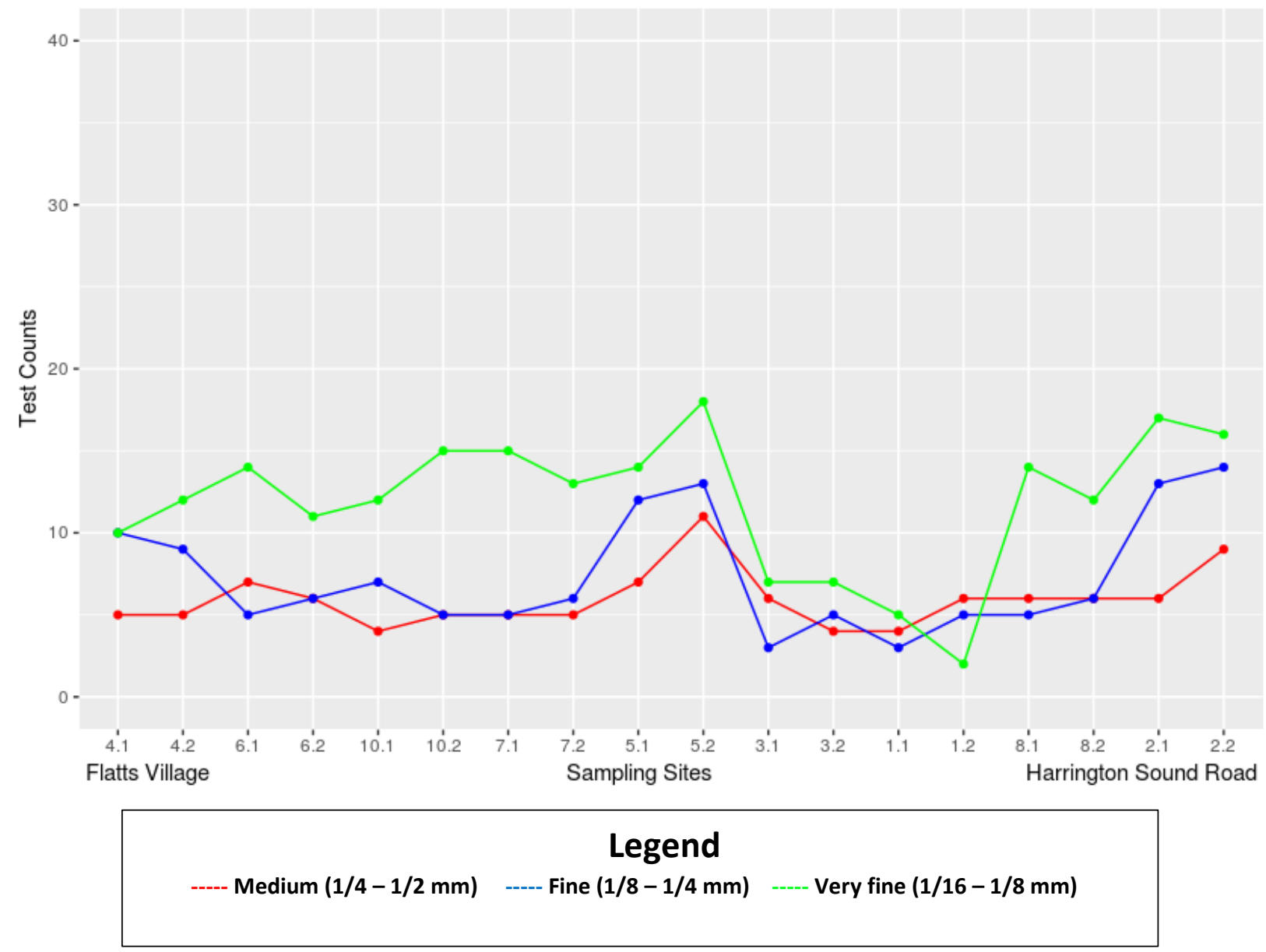

Figure 10. Test count distributions of Elphidium spp. at each sampling locations in different test-size intervals (Medium, Fine, Very fine). 
Ammonia beccarii distribution among sample sites

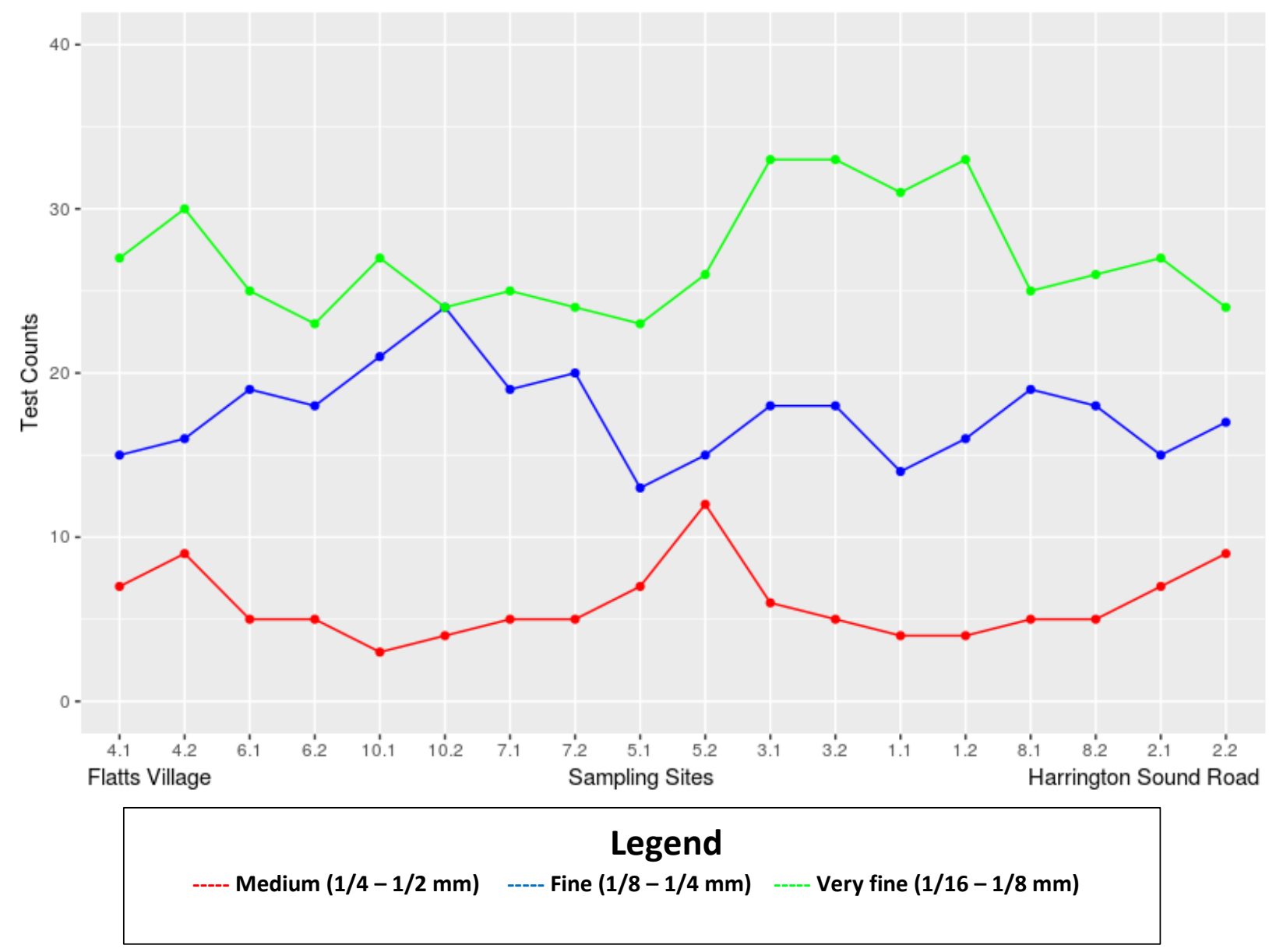

Figure 11. Test count distributions of Ammonia beccarii spp. at each sampling locations in different test-size intervals (Medium, Fine, Very fine). 
A

Coarse test-size fraction of Quinqueloculina \& Triloculina spp. vs Depth

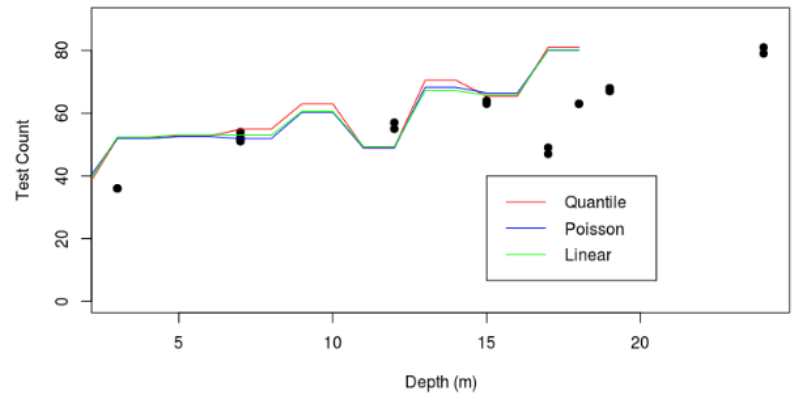

C

Fine test-size fraction of Quinqueloculina \& Triloculina spp. vs Depth

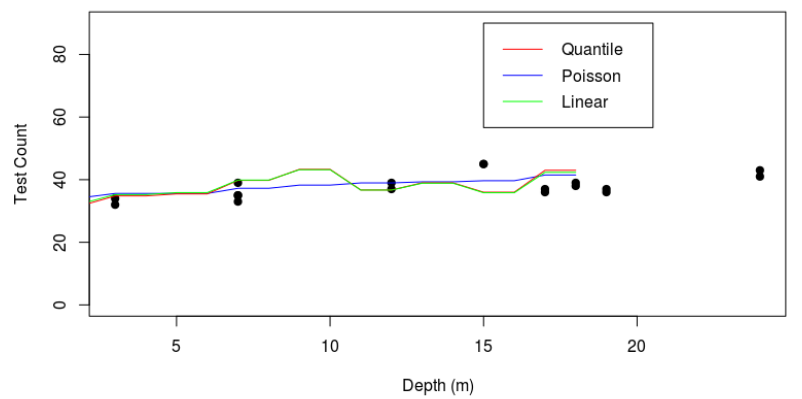

B

Medium test-size fraction of Quinqueloculina \& Triloculina spp. vs Depth

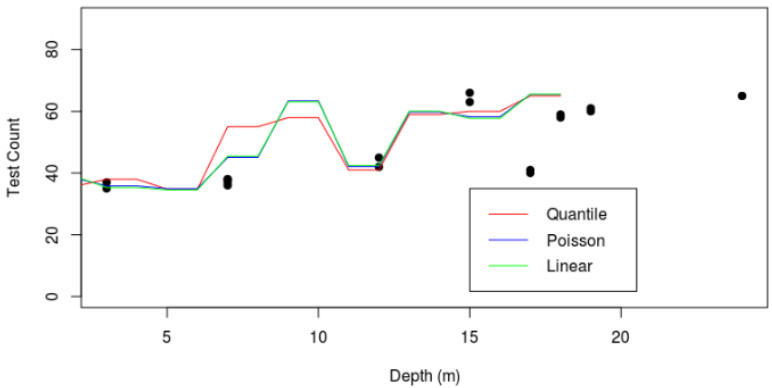

D

Very fine test-size fraction of Quinqueloculina \& Triloculina spp. vs Depth

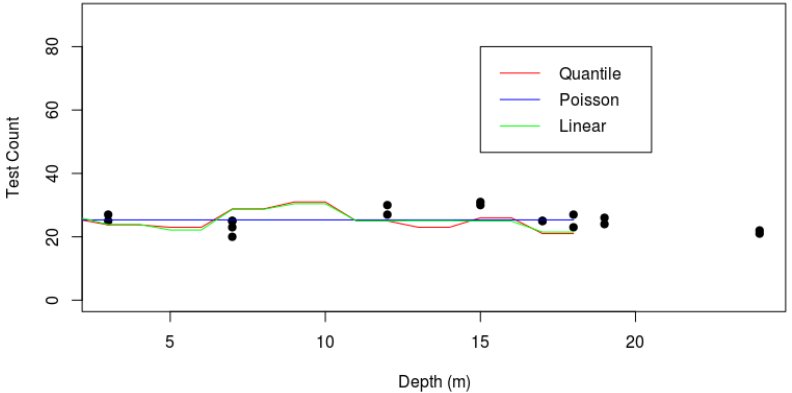

Figure 12. Predictive Models of Quinqueloculina \& Triloculina spp. test counts vs environmental parameters under each test-size interval in scatterplots. (A) Coarse test-size interval; (B) Medium test-size interval; (C) Fine test-size interval; (D) Very fine test-size interval. 
A

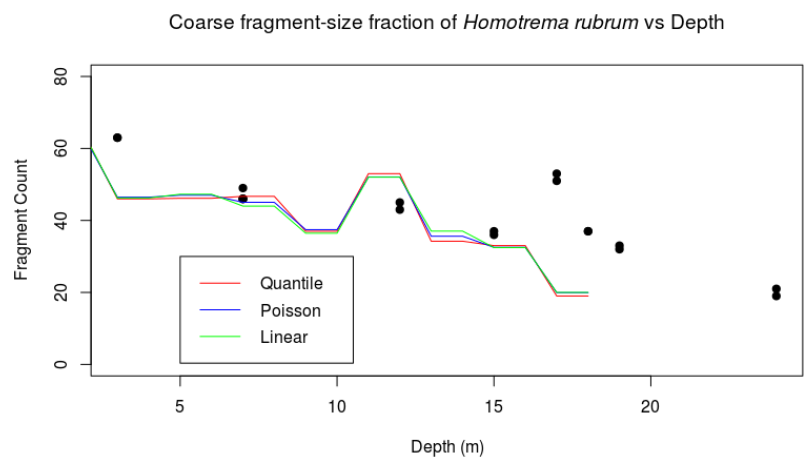

C

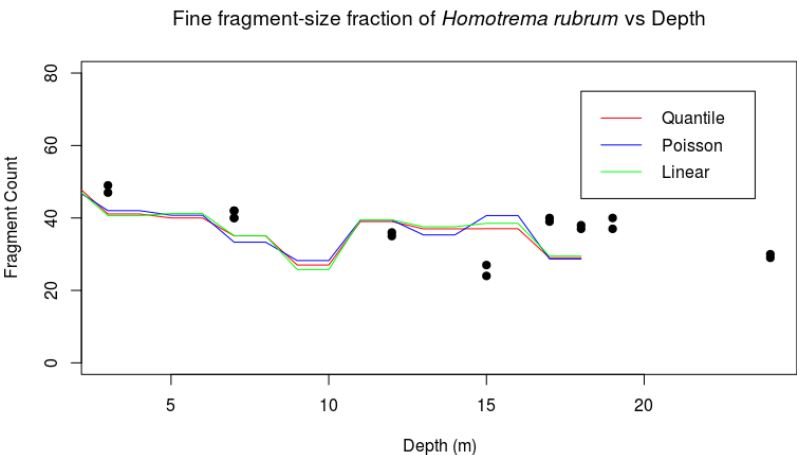

B

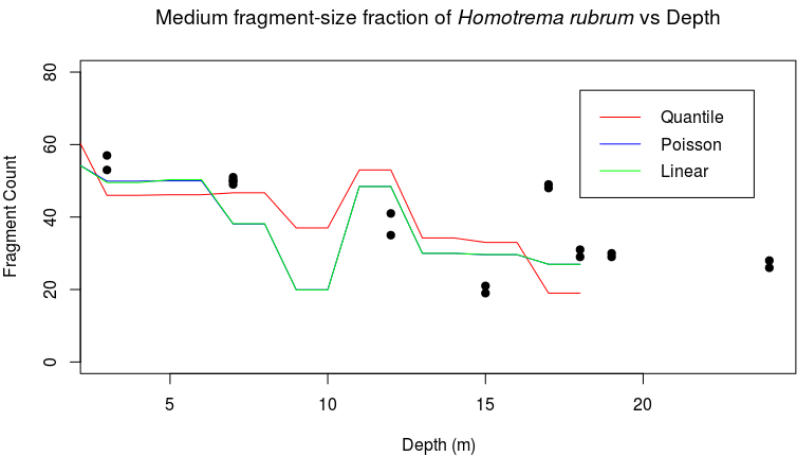

D

Very fine fragment-size fraction of Homotrema rubrum vs Depth

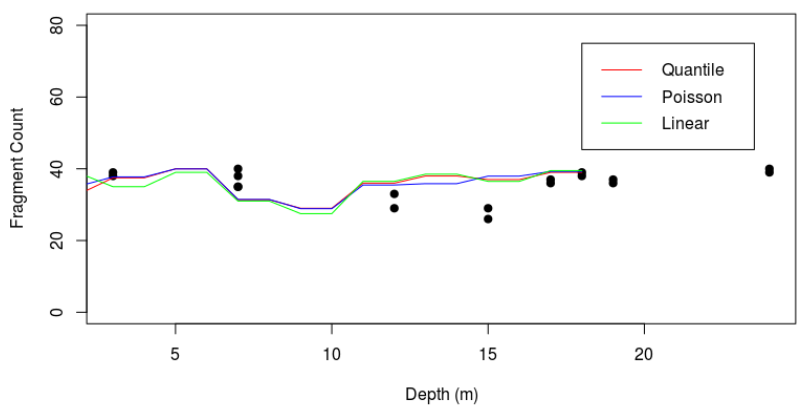

Figure 13. Predictive Models of Homotrema rubrum fragment counts vs environmental parameters at each fragment-size interval in scatterplots. (A) Coarse fragment-size interval; (B) Medium fragment-size interval; (C) Fine fragment-size interval; (D) Very fine fragment-size interval. 
A

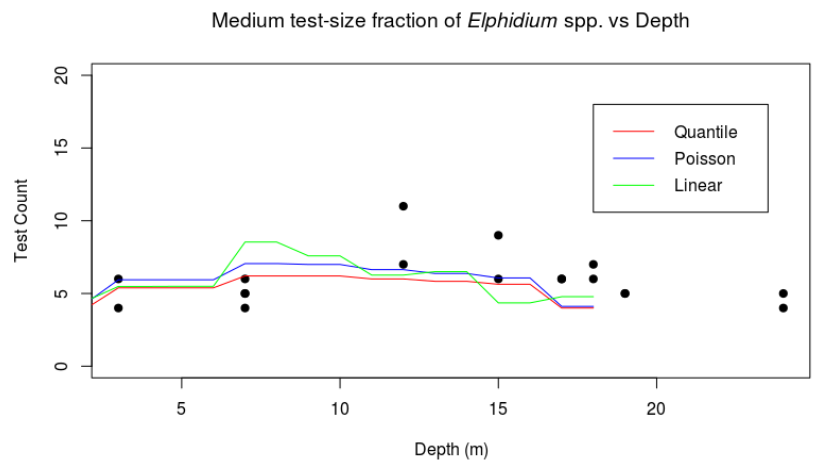

C

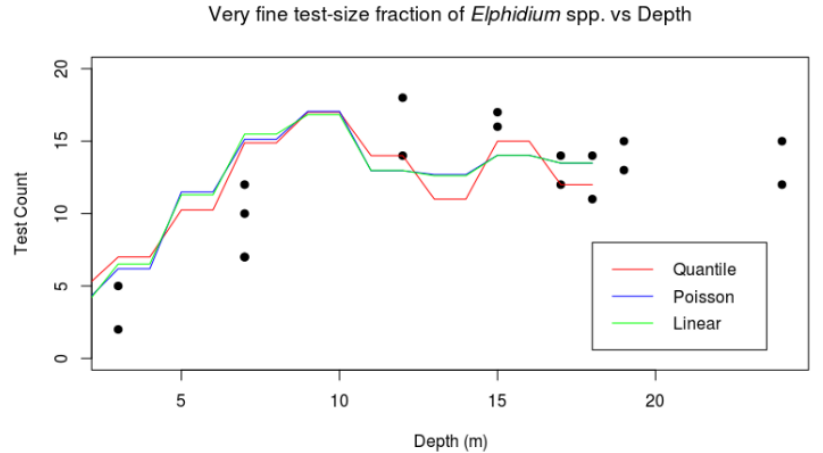

B

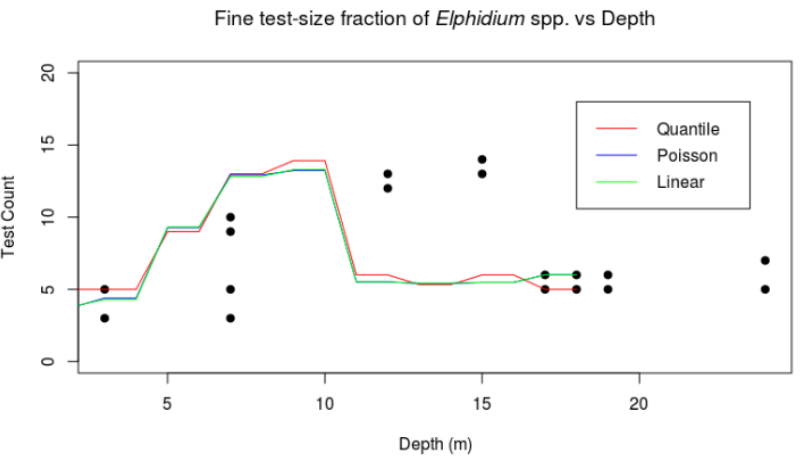

Figure 14. Predictive Models of Elphidium spp. test counts vs environmental parameters at each test-size interval in scatterplots. (A) Medium test-size interval; (B) Fine test-size interval; (C) Very fine test-size interval. 
A

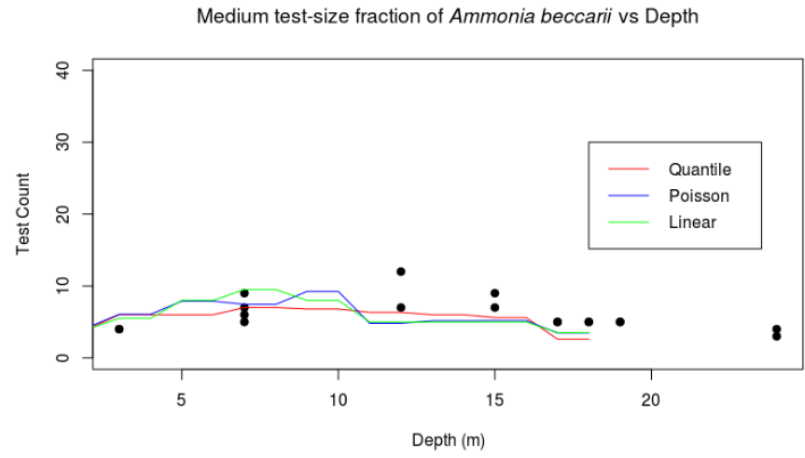

C

Very fine test-size fraction of Ammonia beccarii vs Depth

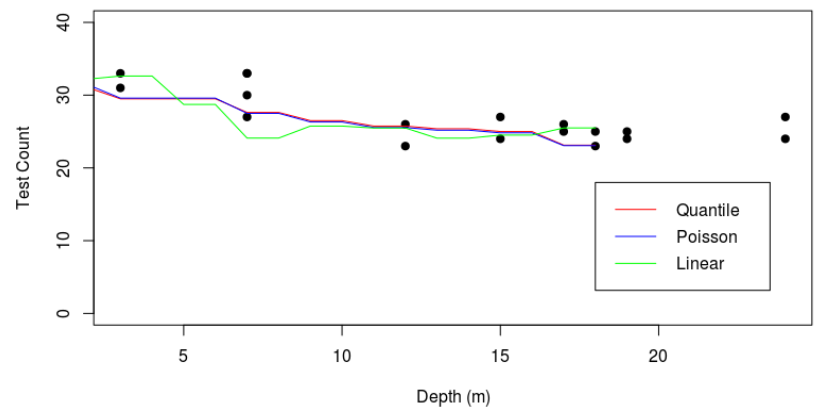

B

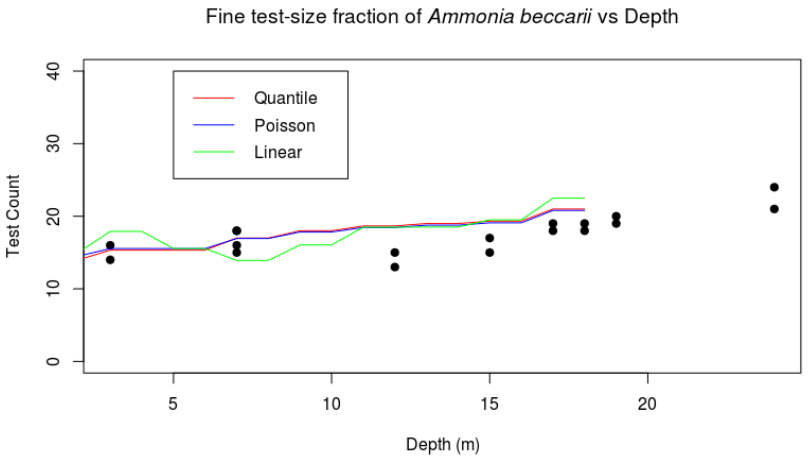

Figure 15. Predictive Models of Ammonia beccarii test counts vs environmental parameters at each test-size interval in scatterplots. (A) Medium test-size interval; (B) Fine test-size interval; (C) Very fine test-size interval. 


\section{Stressplot}

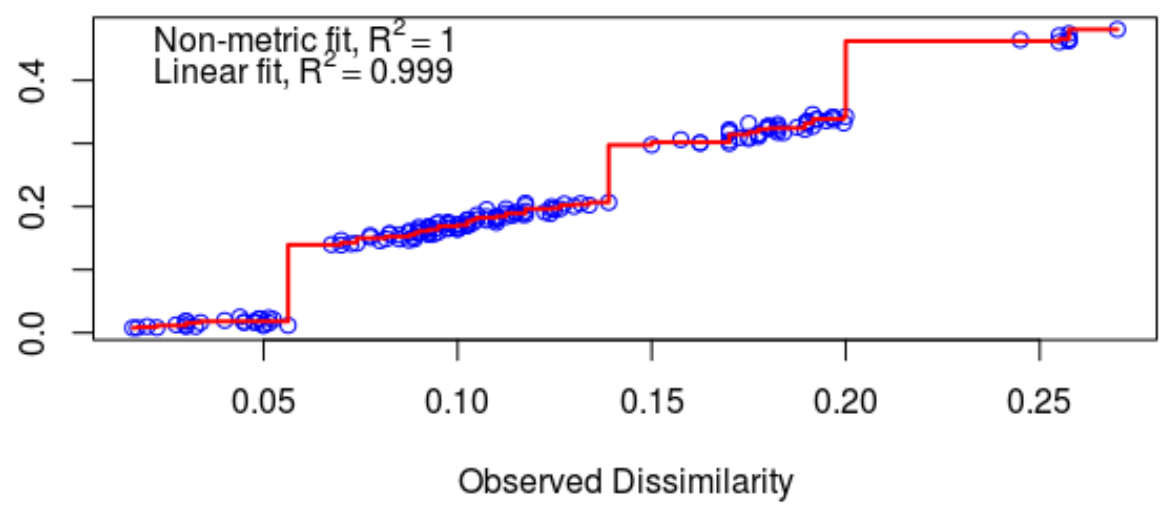

Figure 16. Stress Plot of nMDS, determining compatibility of two dimensions after trial convergence. 


\section{Ordinal Diagram}

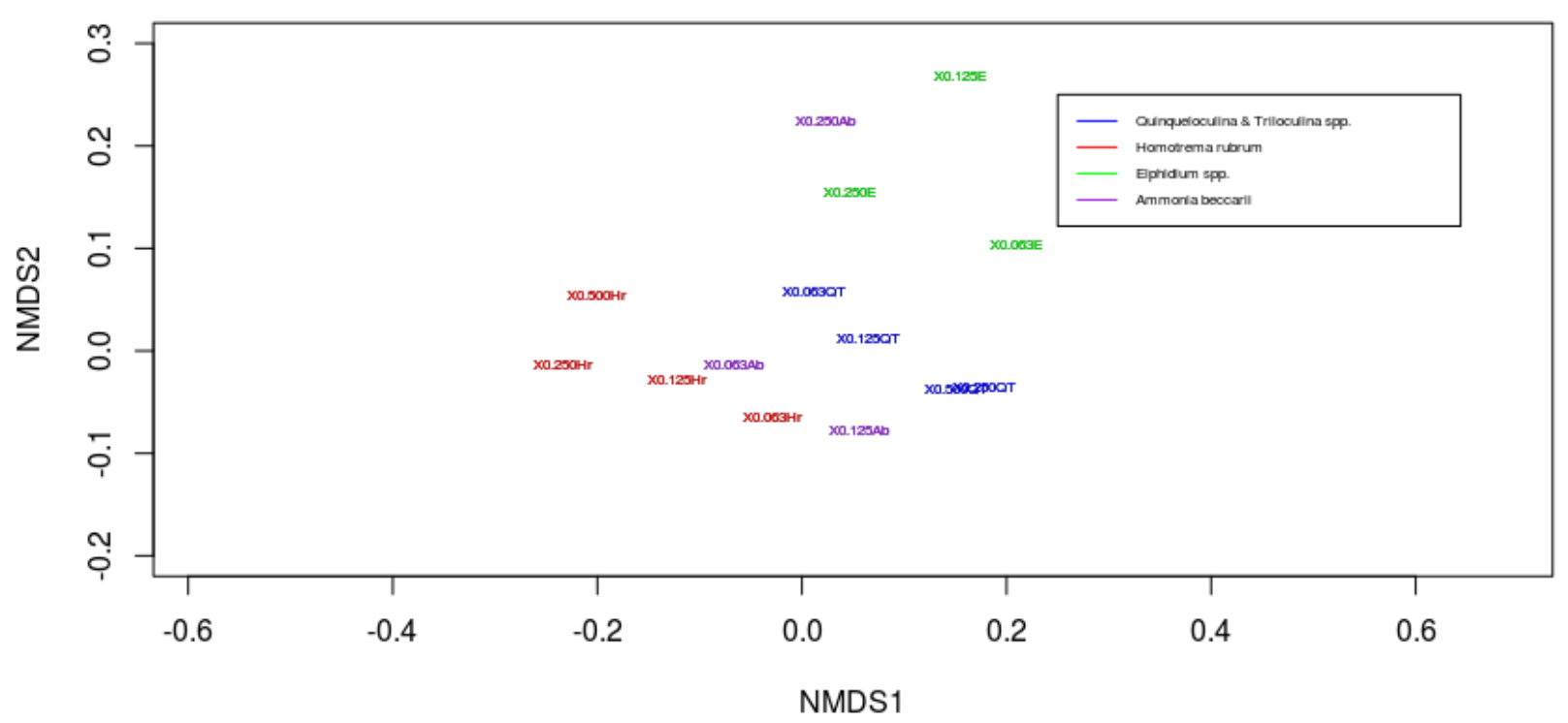

Figure 17. Ordinal plot of taxa groups scaled by Bray Distance and taxa are color-coded. 
A

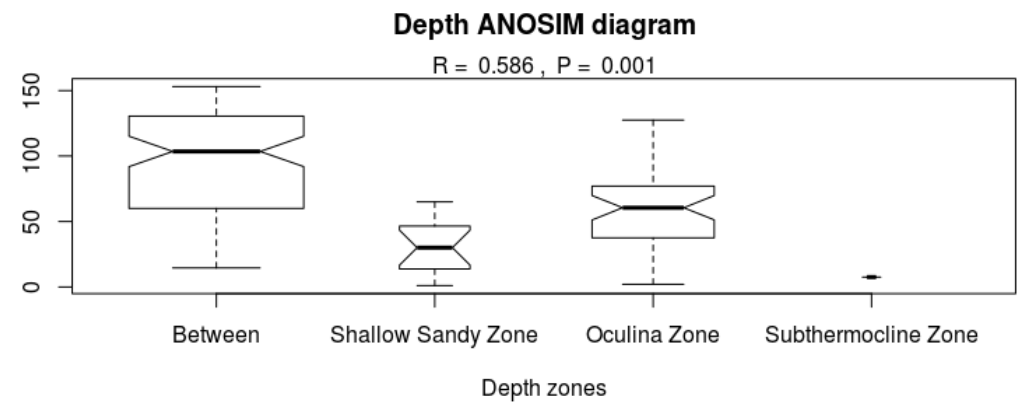

B

Temperature ANOSIM diagram

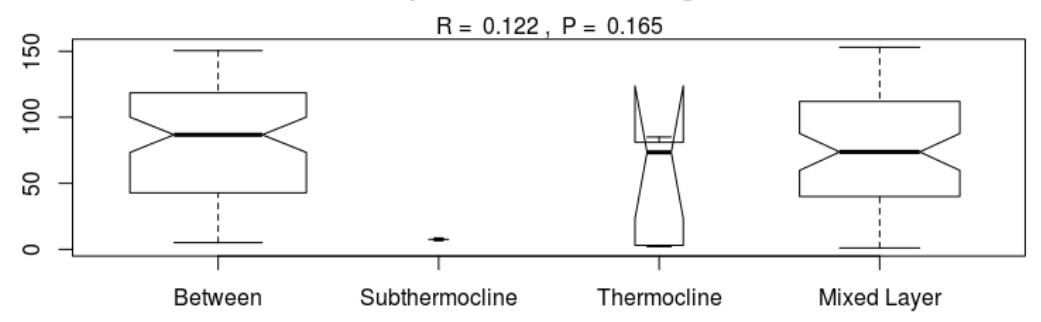

Temperature zones

C

Salinity ANOSIM diagram

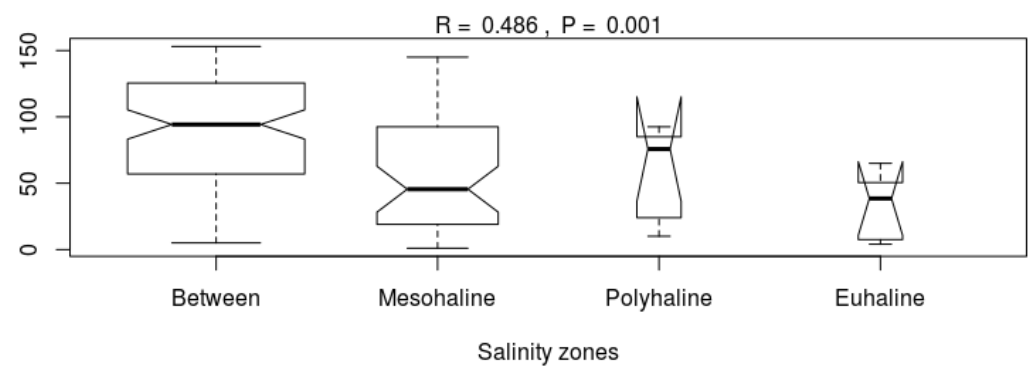

D

RDO ANOSIM diagram

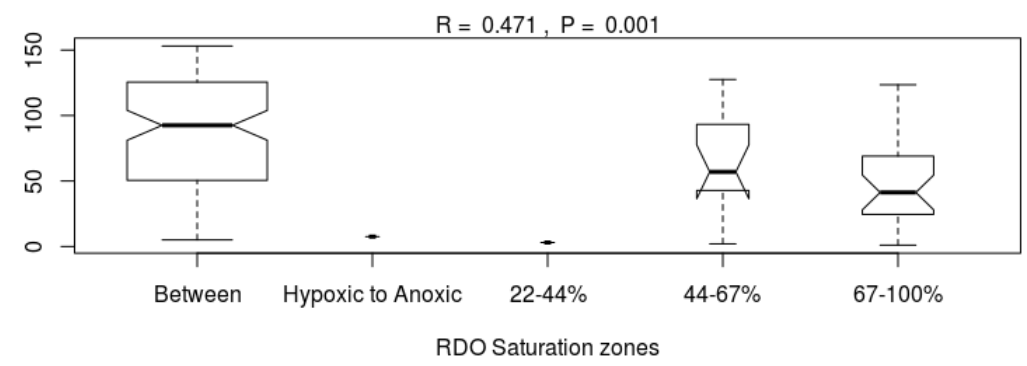

$\mathbf{E}$

pH ANOSIM diagram

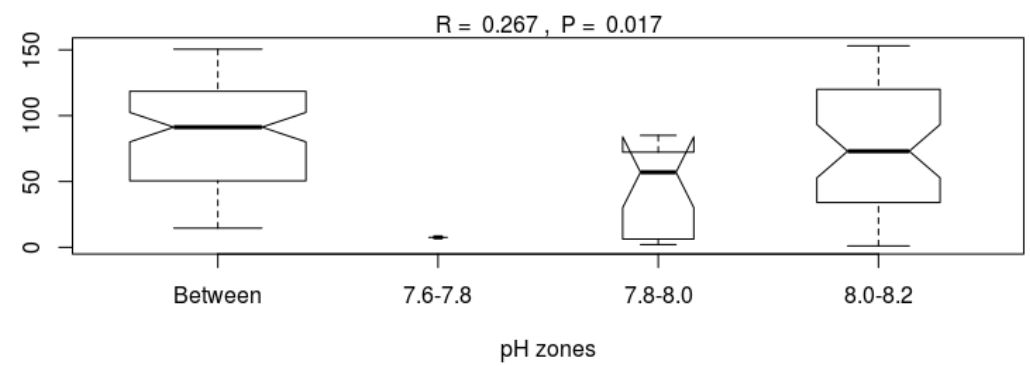

Figure 18. ANOSIM Diagrams of environmental parameters. (A) Depth; (B) Temperature; (C) Salinity; (D)RDO; (E) pH. 


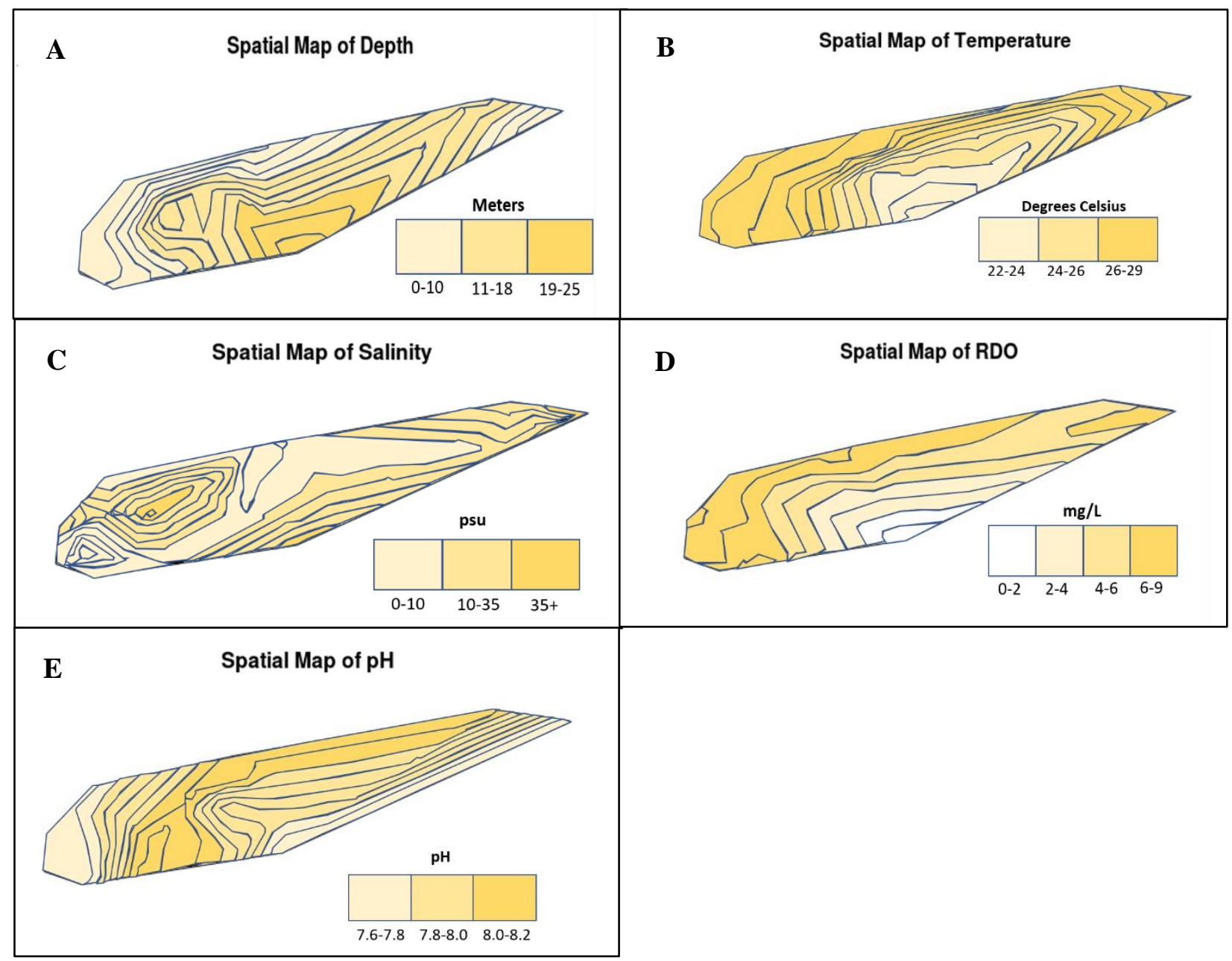

Figure 19. Spatial maps of environmental parameters produced by Additive Quantile Regression Smoothing (AQRS) algorithms. (A) Depth; (B) Temperature; (C) Salinity; (D) Rugged Dissolved Oxygen; (E) pH. 


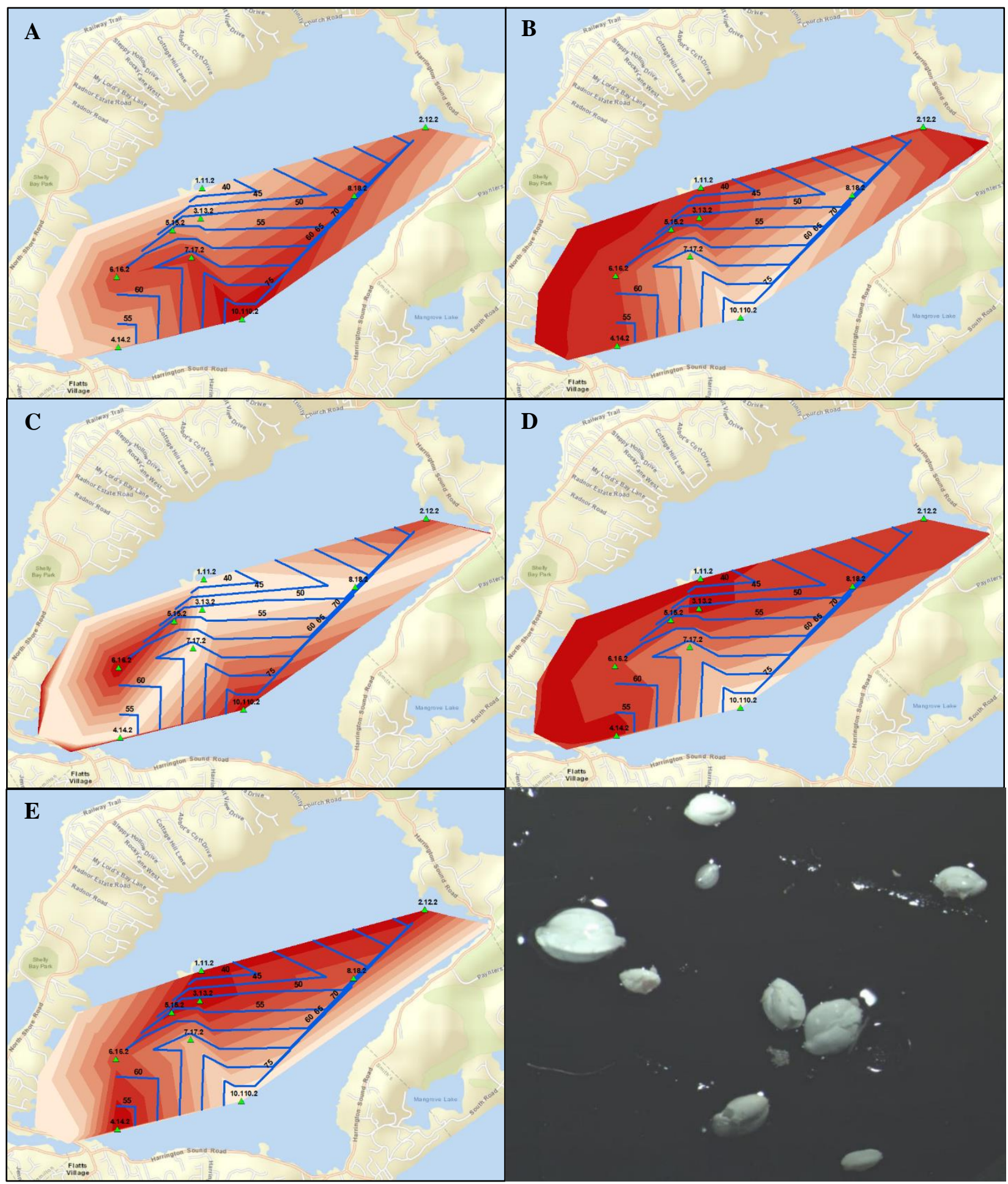

Figure 20. Contour maps of coarse test-size fraction of Quinqueloculina \& Triloculina spp. overlain on environmental parameter spatial maps, produced by ArcMap's Triangulated Irregular Networks (TIN) function. (A) Depth; (B) Temperature; (C) Salinity; (D) Rugged Dissolved Oxygen; (E) pH. 


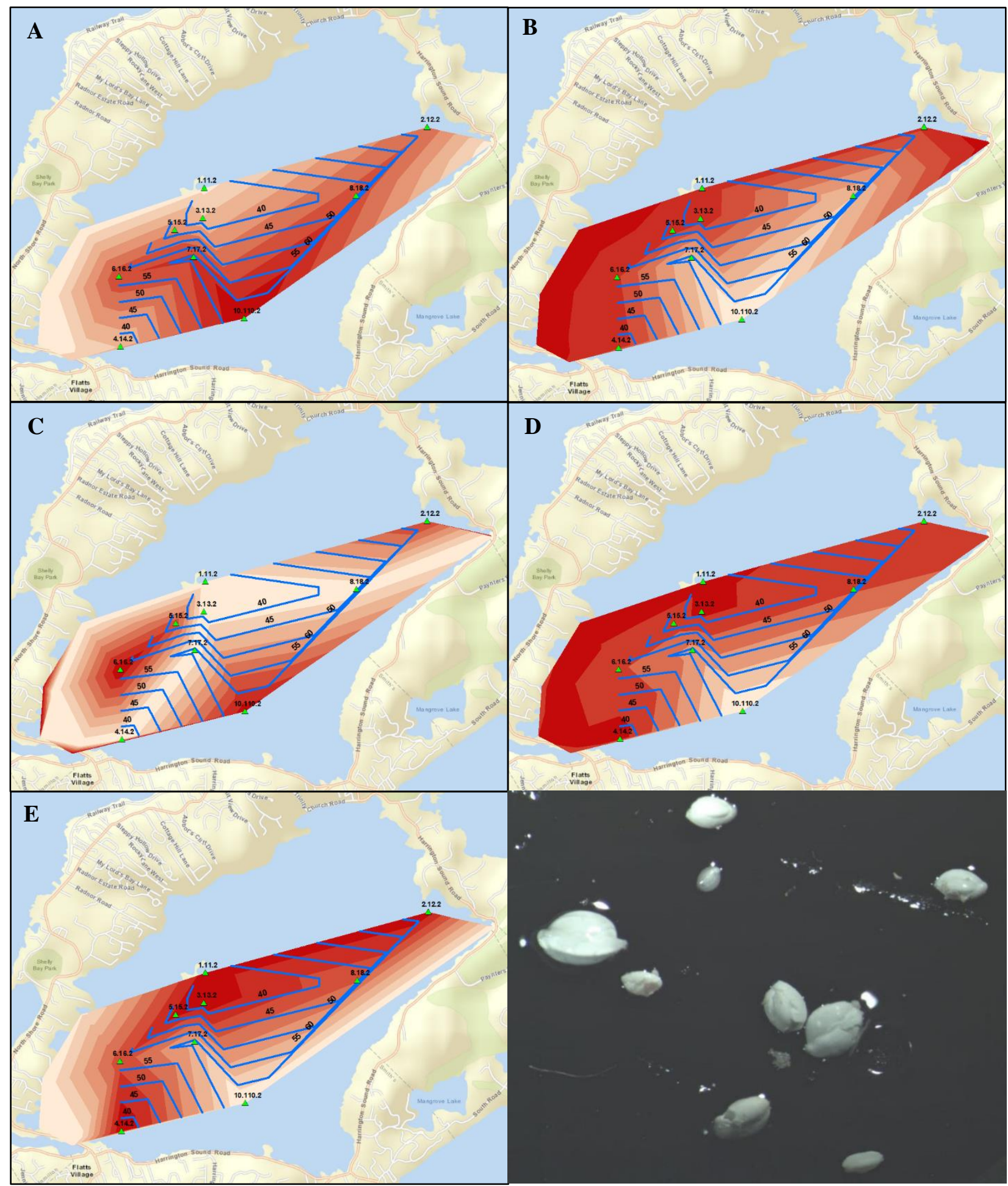

Figure 21. Contour maps of medium test-size fraction of Quinqueloculina \& Triloculina spp. overlain on environmental parameter spatial maps, produced by ArcMap's Triangulated Irregular Networks (TIN) function. (A) Depth; (B) Temperature; (C) Salinity; (D) Rugged Dissolved Oxygen; (E) pH. 


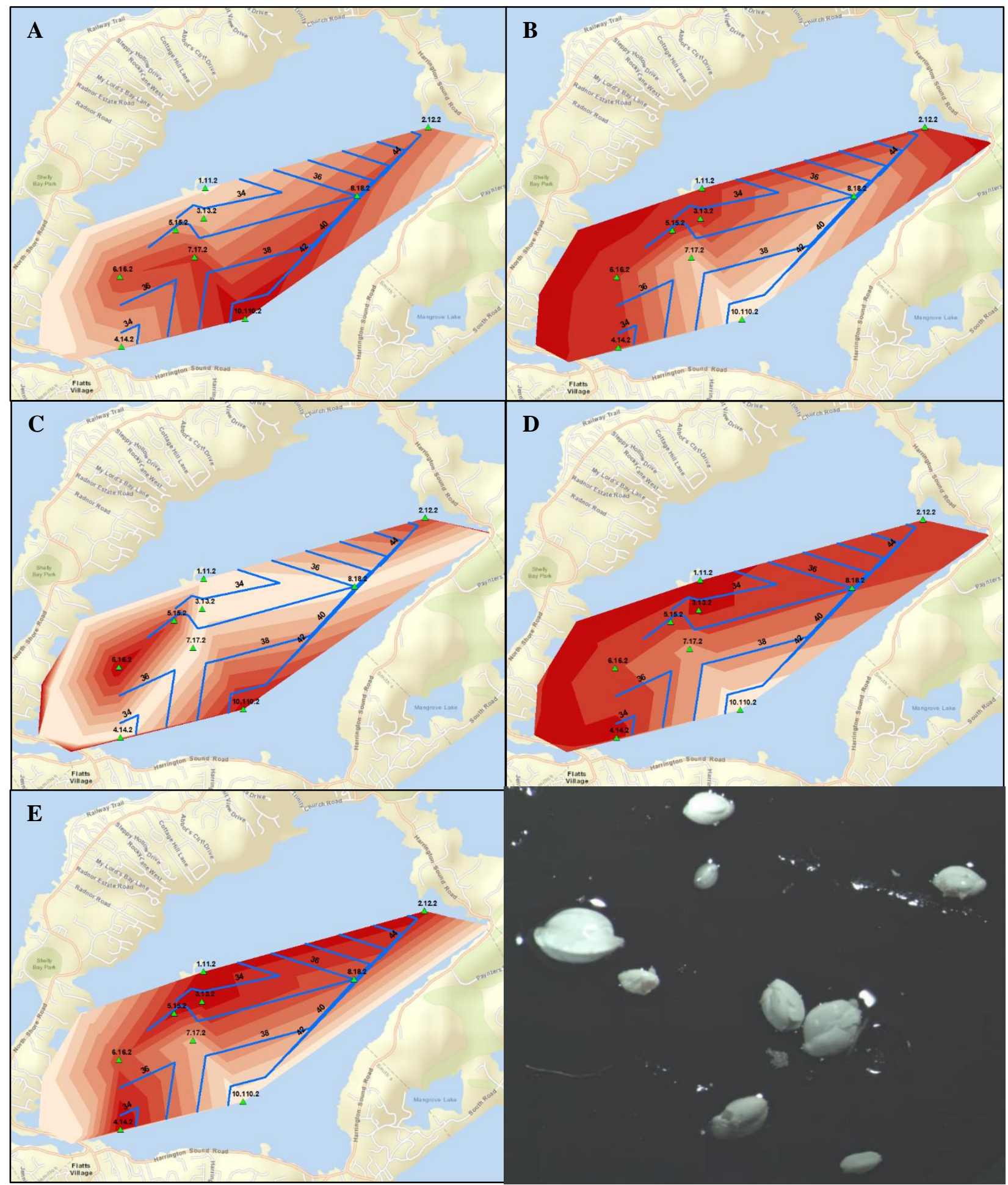

Figure 22. Contour maps of fine test-size fraction of Quinqueloculina \& Triloculina spp. overlain on environmental parameter spatial maps, produced by ArcMap's Triangulated Irregular Networks (TIN) function. (A) Depth; (B) Temperature; (C) Salinity; (D) Rugged Dissolved Oxygen; (E) pH. 


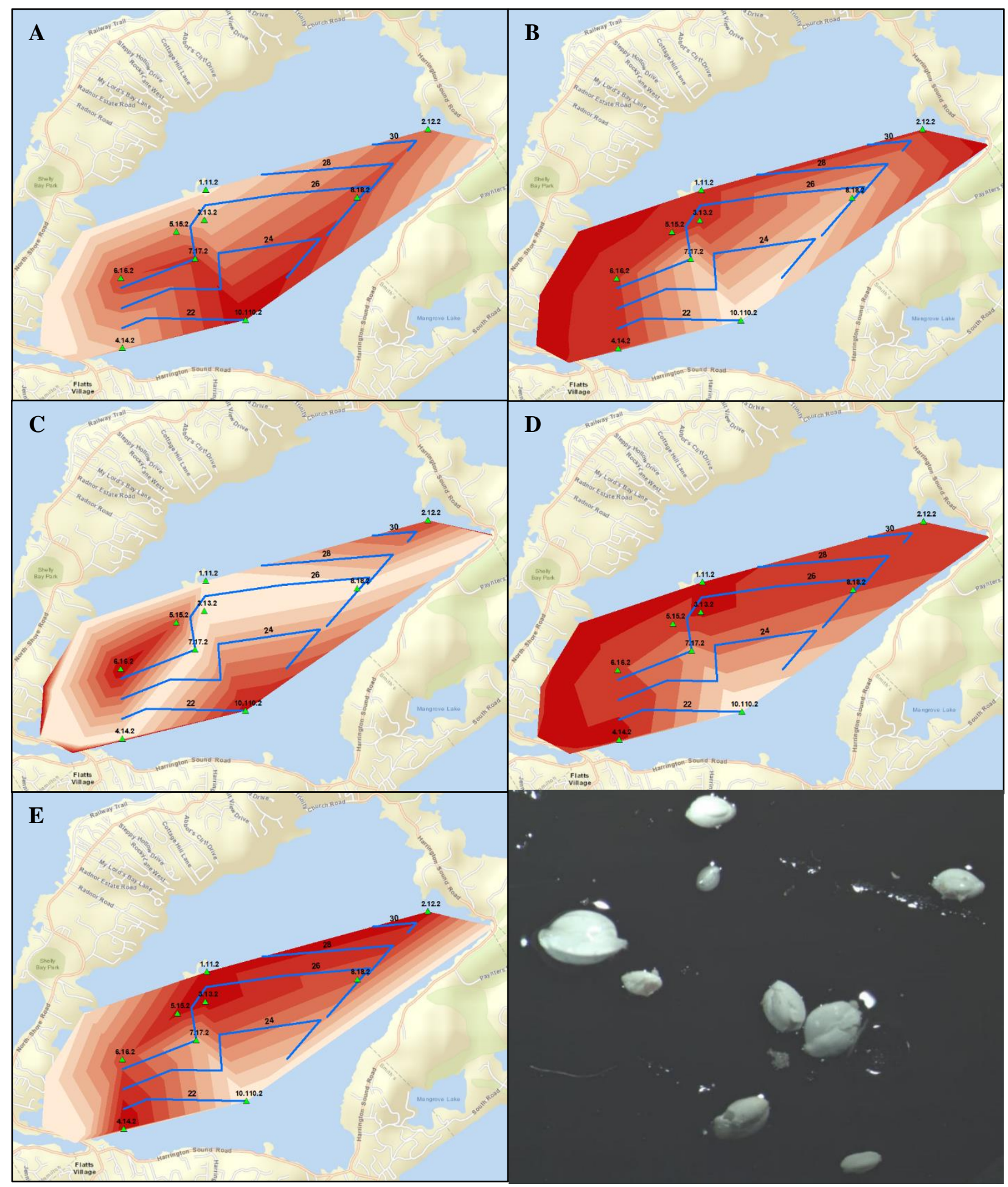

Figure 23. Contour maps of very fine test-size fraction of Quinqueloculina \& Triloculina spp. overlain on environmental parameter spatial maps, produced by ArcMap's Triangulated Irregular Networks (TIN) function. (A) Depth; (B) Temperature; (C) Salinity; (D) Rugged Dissolved Oxygen; (E) pH. 


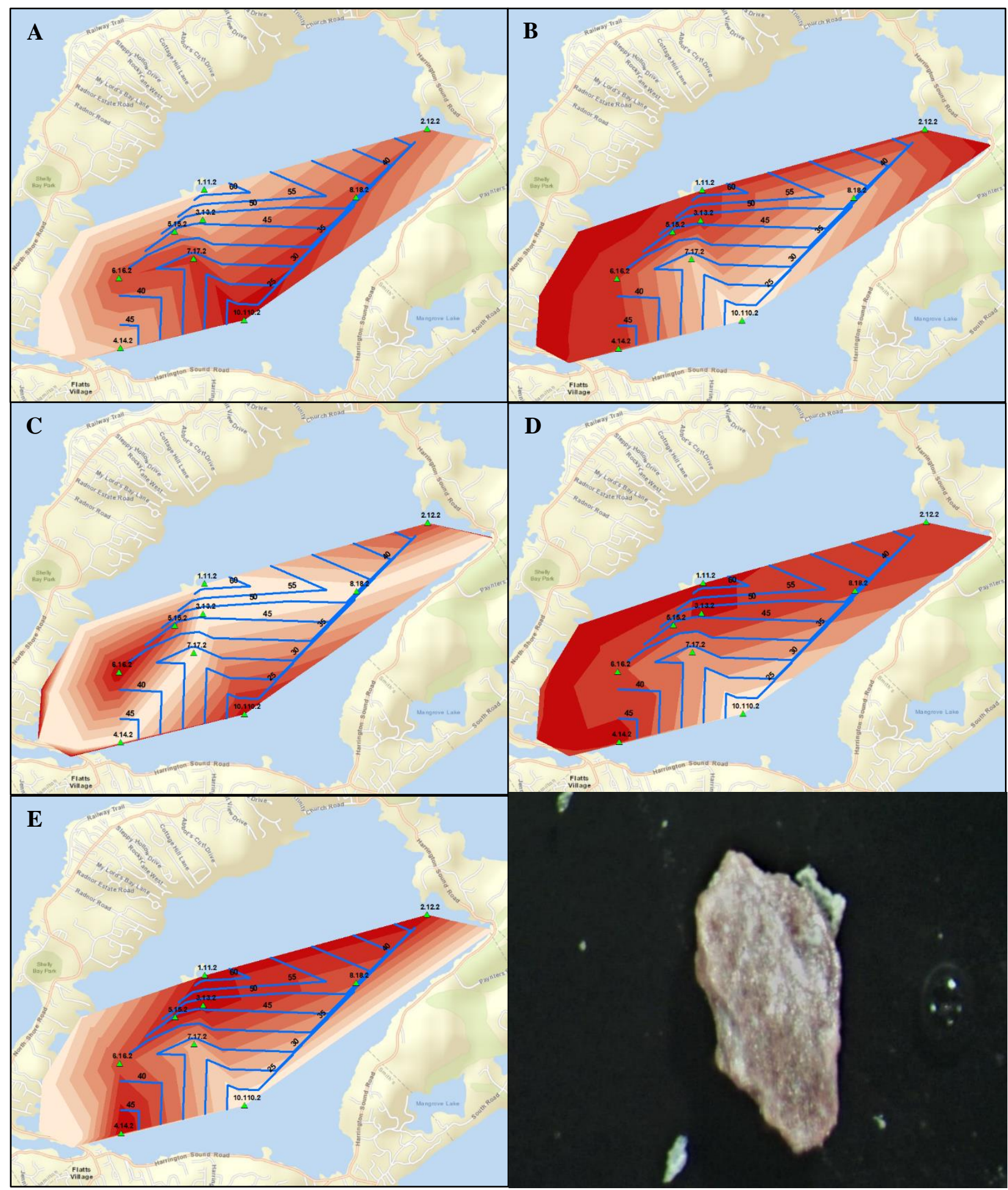

Figure 24. Contour maps of coarse fragment-size fraction of Homotrema rubrum fragments overlain on environmental parameter spatial maps, produced by ArcMap's Triangulated Irregular Networks (TIN) function. (A) Depth; (B) Temperature; (C) Salinity; (D) Rugged Dissolved Oxygen; (E) pH. 


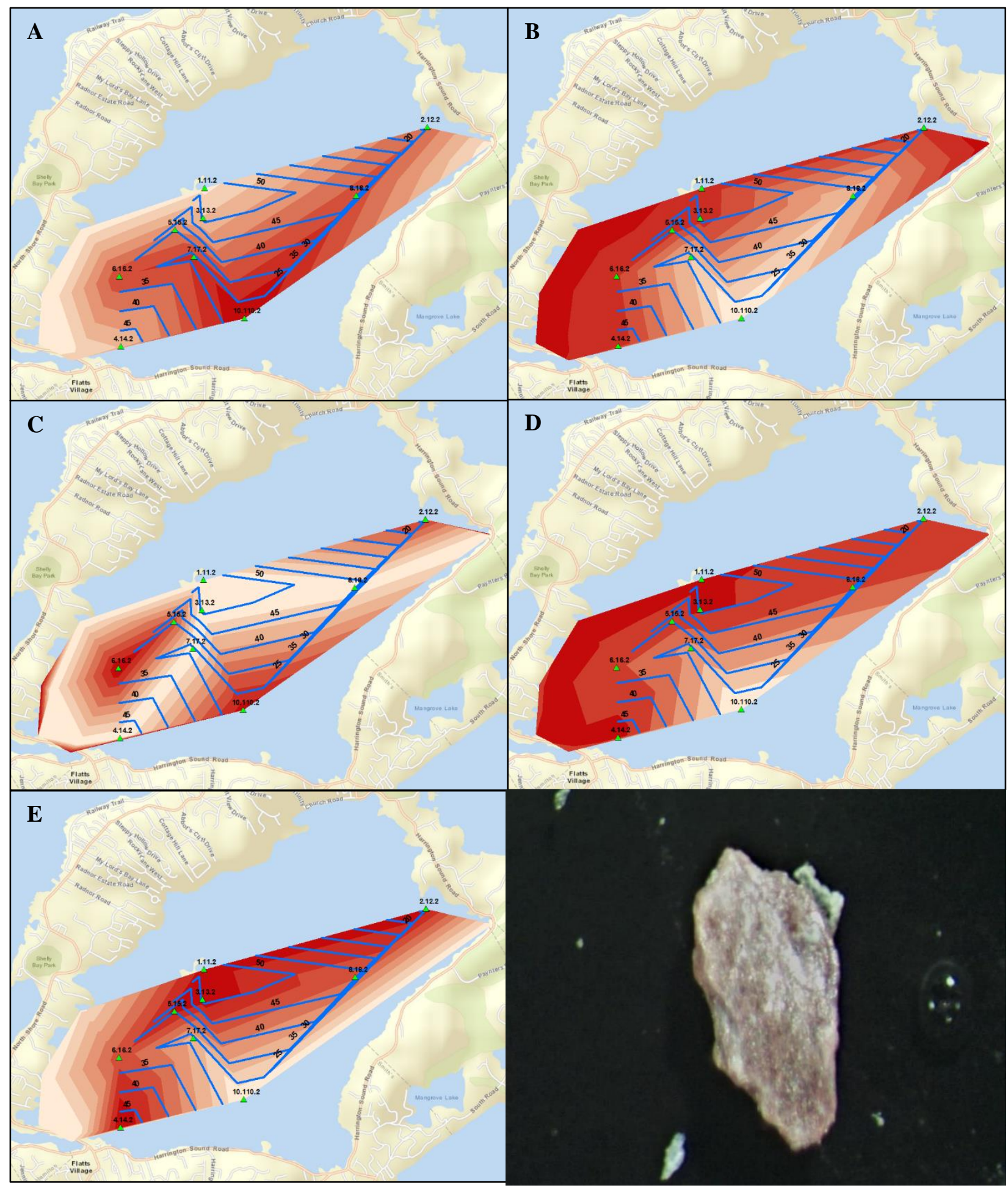

Figure 25. Contour maps of medium fragment-size fraction of Homotrema rubrum fragments overlain on spatial maps of environmental parameter, produced by ArcMap's Triangulated Irregular Networks (TIN) function. (A) Depth; (B) Temperature; (C) Salinity; (D) Rugged Dissolved Oxygen; (E) pH. 


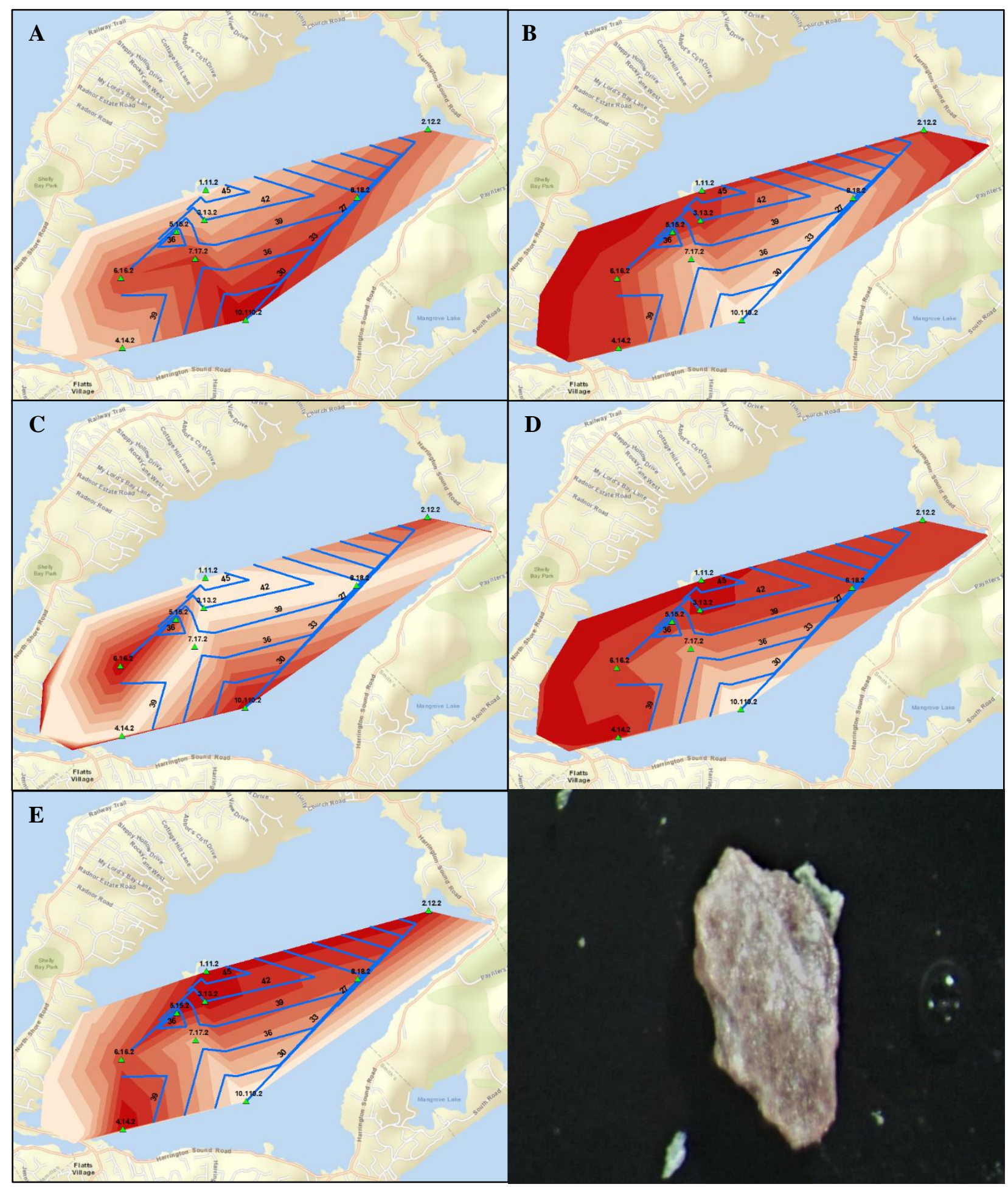

Figure 26. Contour maps of fine fragment-size fraction of Homotrema rubrum fragments overlain on spatial maps of environmental parameter, produced by ArcMap's Triangulated Irregular Networks (TIN) function. (A) Depth; (B) Temperature; (C) Salinity; (D) Rugged Dissolved Oxygen; (E) pH. 


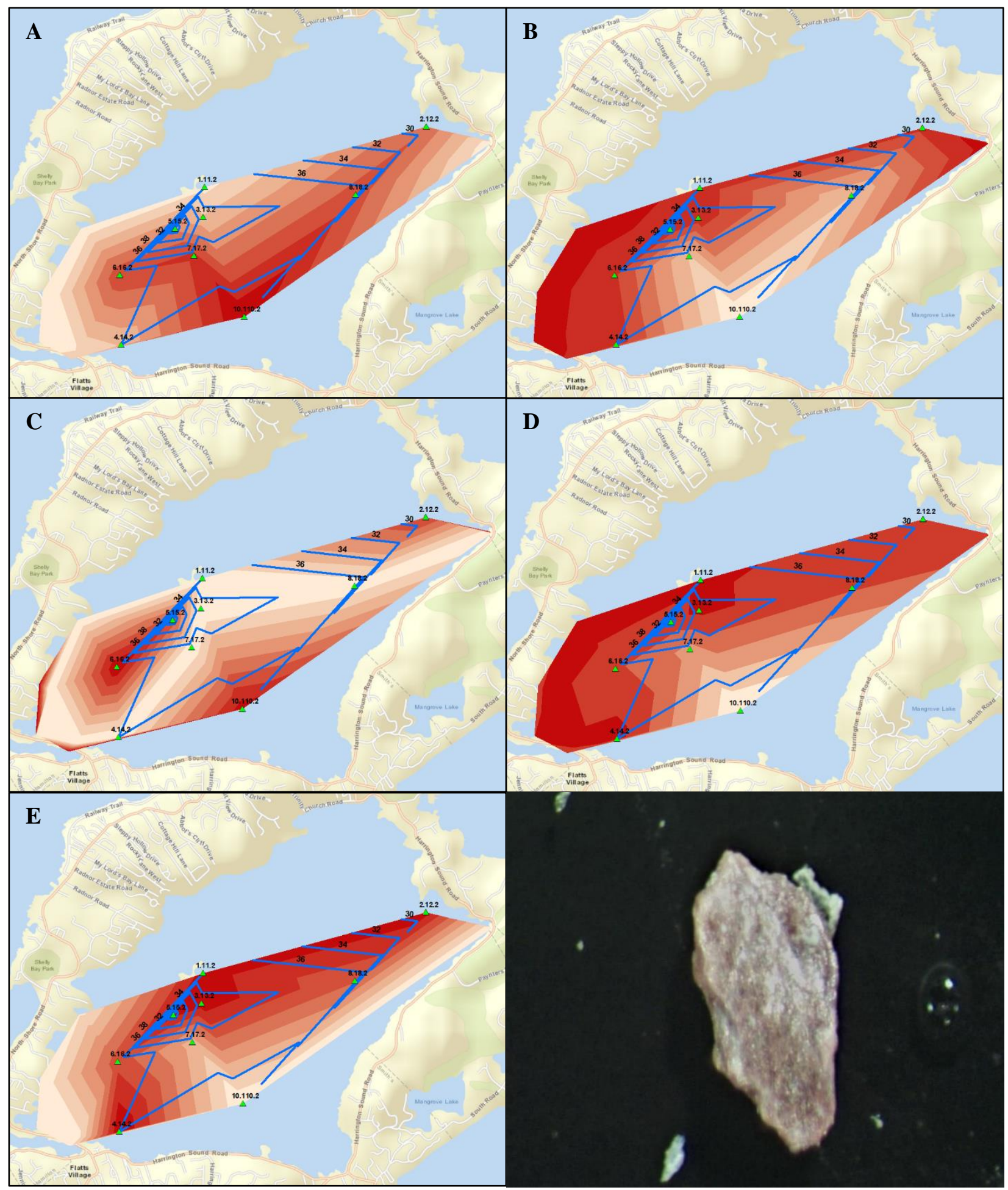

Figure 27. Contour maps of very fine fragment-size fraction of Homotrema rubrum fragments overlain on spatial maps of environmental parameter, produced by ArcMap's Triangulated Irregular Networks (TIN) function. (A) Depth; (B) Temperature; (C) Salinity; (D) Rugged Dissolved Oxygen; (E) pH. 


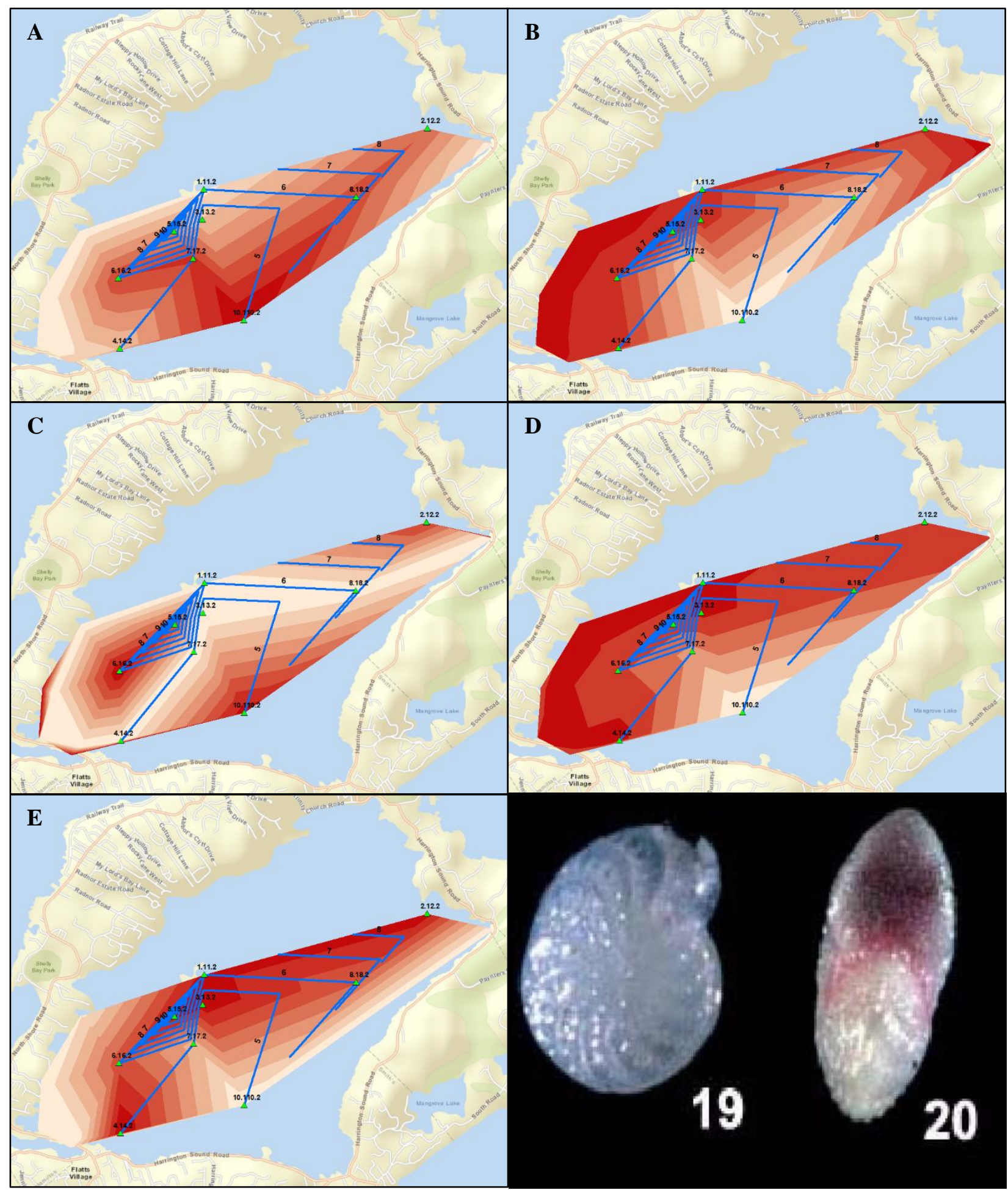

Figure 28. Contour maps of medium test-size fraction of Elphidium spp. overlain on spatial maps of environmental parameter, produced by ArcMap's Triangulated Irregular Networks (TIN) function. (A) Depth; (B) Temperature; (C) Salinity; (D) Rugged Dissolved Oxygen; (E) pH. 


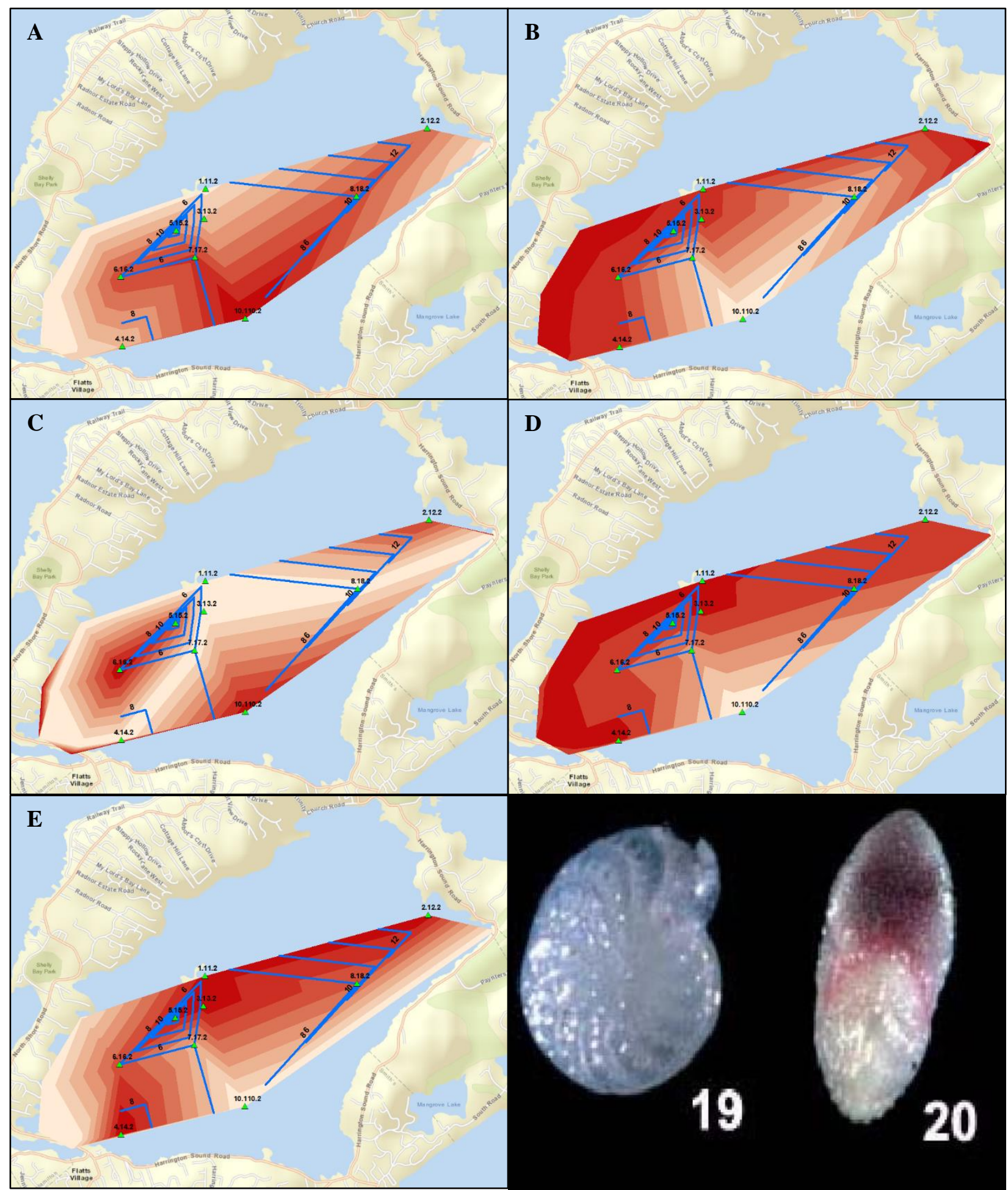

Figure 29. Contour maps of fine test-size fraction Elphidium spp. overlain on spatial maps of environmental parameter, produced by ArcMap's Triangulated Irregular Networks (TIN) function. (A) Depth; (B) Temperature; (C) Salinity; (D) Rugged Dissolved Oxygen; (E) pH. 


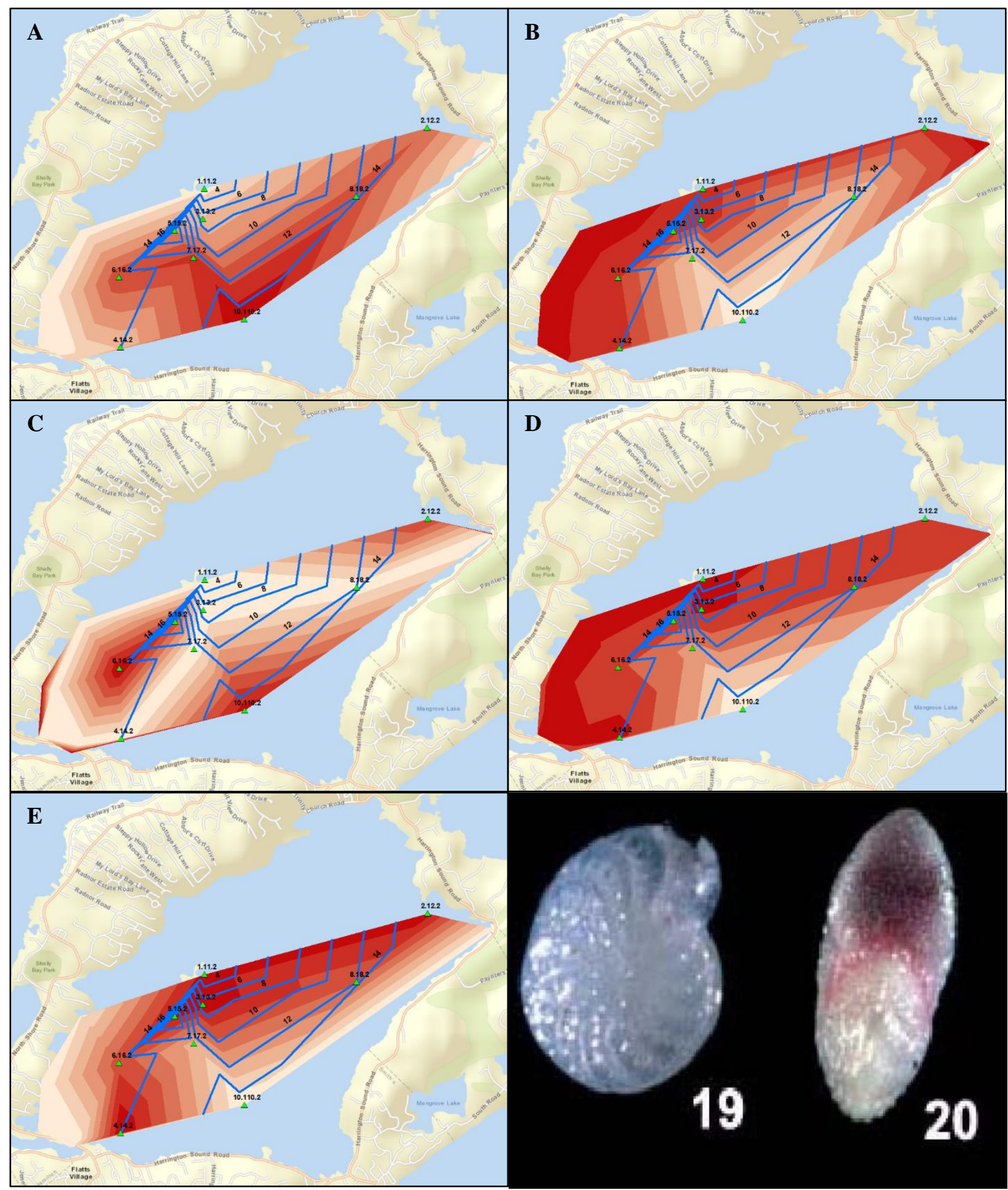

Figure 30. Contour maps of very fine test-size fraction Elphidium spp. overlain on spatial maps of environmental parameter, produced by ArcMap's Triangulated Irregular Networks (TIN) function. (A) Depth; (B) Temperature; (C) Salinity; (D) Rugged Dissolved Oxygen; (E) pH. 


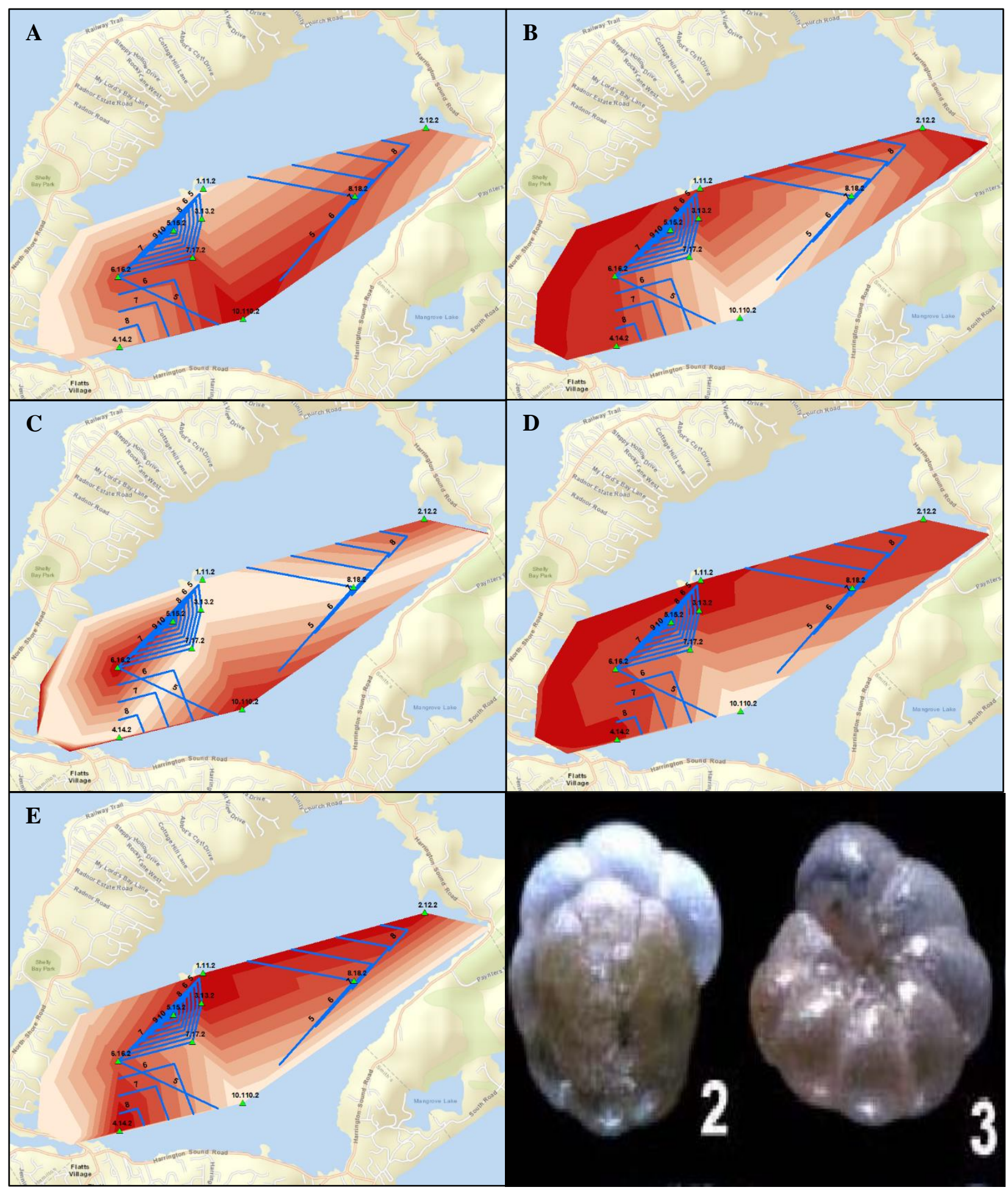

Figure 31. Contour maps of medium test-size fraction Ammonia beccarii overlain on spatial maps of environmental parameter, produced by ArcMap's Triangulated Irregular Networks (TIN) function. (A) Depth; (B) Temperature; (C) Salinity; (D) Rugged Dissolved Oxygen; (E) pH. 


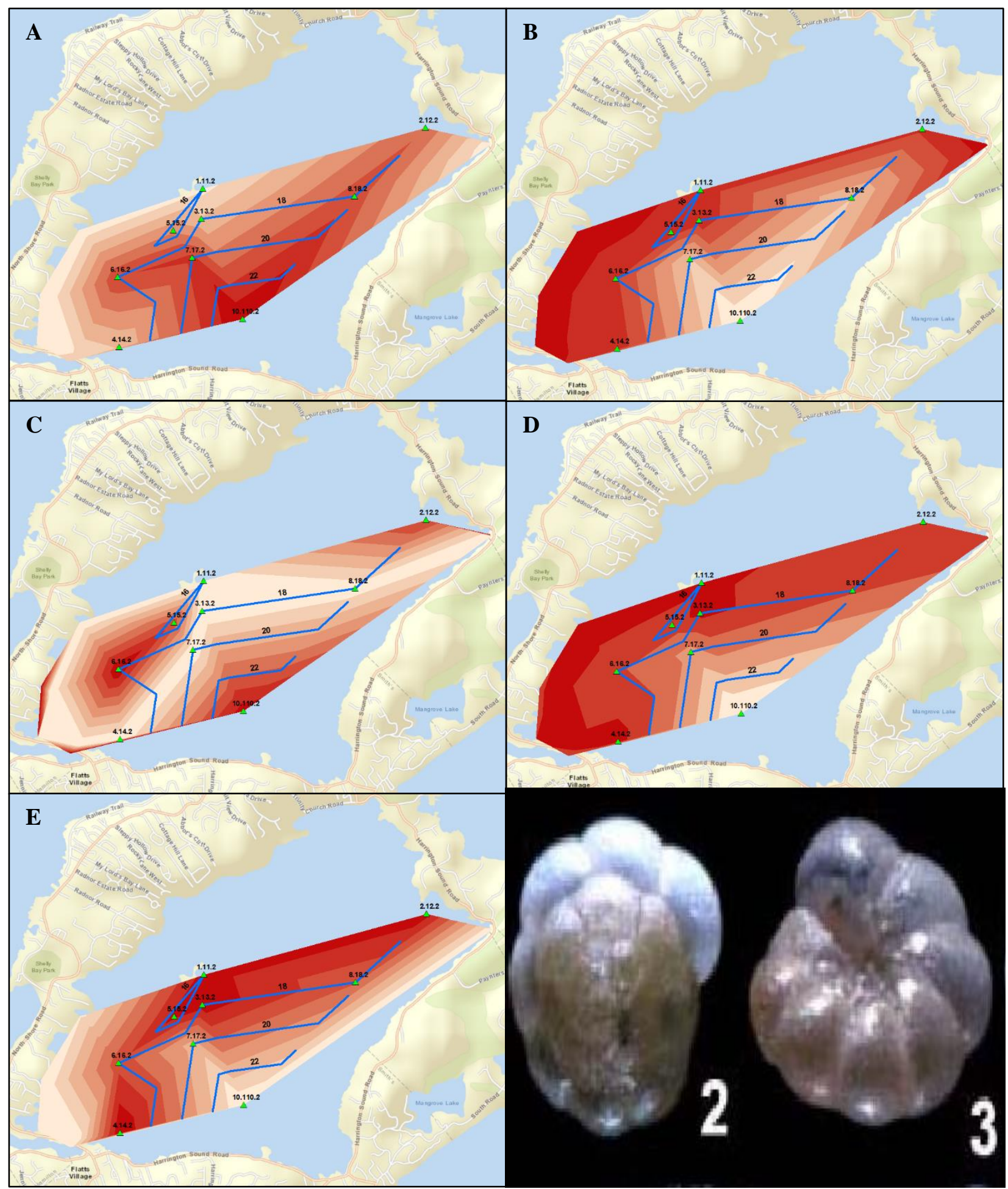

Figure 32. Contour maps of fine test-size fraction Ammonia beccarii overlain on spatial maps of environmental parameter, produced by ArcMap's Triangulated Irregular Networks (TIN) function. (A) Depth; (B) Temperature; (C) Salinity; (D) Rugged Dissolved Oxygen; (E) pH. 


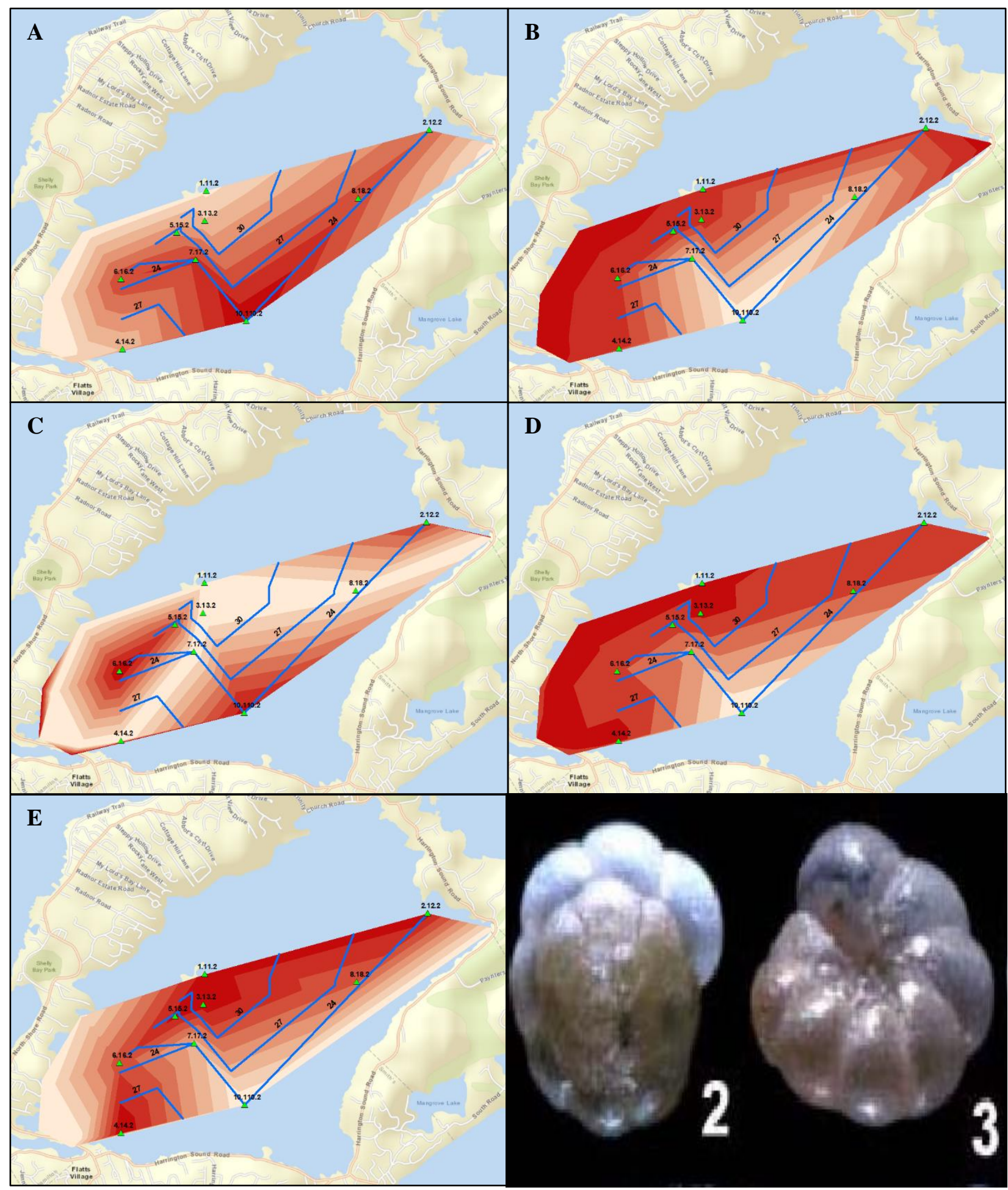

Figure 33. Contour maps of very fine test-size fraction Ammonia beccarii overlain on spatial maps of environmental parameter, produced by ArcMap's Triangulated Irregular Networks (TIN) function. (A) Depth; (B) Temperature; (C) Salinity; (D) Rugged Dissolved Oxygen; (E) pH. 
Table 1. Shapiro-Wilk Normality test results for all taxa under each test/fragment-size interval. Green cells indicate failure to reject $\mathrm{Ho}_{\mathrm{o}}$ while pink cells indicate rejection of $\mathbf{H}_{0}$.

\begin{tabular}{|c|c|c|c|c|}
\hline Taxa & $\begin{array}{l}\text { Test/Fragment- } \\
\text { size interval }\end{array}$ & W-Statistic & p-value & Conclusion \\
\hline $\begin{array}{l}\text { Quinqueloculina \& } \\
\text { Triloculina spp. }\end{array}$ & Coarse & 0.962 & 0.632 & Fails to reject $\mathbf{H}_{0}$ \\
\hline $\begin{array}{l}\text { Quinqueloculina \& } \\
\text { Triloculina spp. }\end{array}$ & Medium & 0.822 & 0.003 & Rejects $\mathrm{H}_{\mathrm{o}}$ \\
\hline $\begin{array}{l}\text { Quinqueloculina \& } \\
\text { Triloculina spp. }\end{array}$ & Fine & 0.943 & 0.331 & Fails to reject $\mathrm{H}_{\mathrm{o}}$ \\
\hline $\begin{array}{l}\text { Quinqueloculina \& } \\
\text { Triloculina spp. }\end{array}$ & Very Fine & 0.959 & 0.583 & Fails to reject $\mathbf{H}_{o}$ \\
\hline Homotrema rubrum & Coarse & 0.961 & 0.619 & Fails to reject $\mathrm{H}_{\mathbf{0}}$ \\
\hline Homotrema rubrum & Medium & 0.902 & 0.061 & Fails to reject $\mathrm{H}_{0}$ \\
\hline Homotrema rubrum & Fine & 0.950 & 0.433 & Fails to reject $H_{0}$ \\
\hline Homotrema rubrum & Very Fine & 0.849 & 0.008 & Rejects $\mathrm{H}_{0}$ \\
\hline Elphidium spp. & Medium & 0.830 & 0.004 & Rejects $\mathrm{H}_{0}$ \\
\hline Elphidium spp. & Fine & 0.853 & 0.010 & Rejects $\mathrm{H}_{0}$ \\
\hline Elphidium spp. & Very Fine & 0.935 & 0.239 & Fails to reject $\mathrm{H}_{0}$ \\
\hline Ammonia beccarii & Medium & 0.860 & 0.012 & Rejects $\mathrm{H}_{0}$ \\
\hline Ammonia beccarii & Fine & 0.963 & 0.661 & Fails to reject $\mathbf{H}_{0}$ \\
\hline Ammonia beccarii & Very Fine & 0.860 & 0.012 & Rejects $\mathrm{H}_{\mathrm{o}}$ \\
\hline \multicolumn{2}{|c|}{$\begin{array}{l}\text { Ho: The population is normally distributed } \\
\text { (Gaussian) }\end{array}$} & \multicolumn{3}{|c|}{$\begin{array}{l}\text { Ha: The population is not normally distributed } \\
\text { (Quantile/Non-parametric) }\end{array}$} \\
\hline
\end{tabular}


Table 2. Predictive Models of Quinqueloculina \& Triloculina spp. test counts vs environmental parameters under each test-size interval, including color-coded inclusion-exclusion and formula.

\begin{tabular}{|c|c|c|c|c|c|c|c|c|}
\hline Taxa & Regression & $\begin{array}{l}\text { Test-size } \\
\text { Interval }\end{array}$ & Depth & Temperature & Salinity & RDO & pH & Formula \\
\hline $\begin{array}{l}\text { QT } \\
\text { spp. }\end{array}$ & Quantile & Coarse & & & & & & $\begin{array}{l}\text { Species Count } \sim 37.87 \text { (Depth) } \\
+32.93 \text { (Temperature) + - } \\
0.618(\text { Salinity) +- } \\
\text { 1.22081(Depth:Temperature) + } \\
-891.55291\end{array}$ \\
\hline $\begin{array}{l}\text { QT } \\
\text { spp. }\end{array}$ & Poisson & Coarse & & & & & & $\begin{array}{l}\text { Species Count } \sim 0.688(\text { Depth }) \\
+0.606(\text { Temperature })+- \\
0.014 \text { (Salinity) +- } \\
0.022(\text { Depth:Temperature }+- \\
13.44\end{array}$ \\
\hline $\begin{array}{l}\text { QT } \\
\text { spp. }\end{array}$ & Linear & Coarse & & & & & & $\begin{array}{l}\text { Species Count } \sim 38.83(\text { Depth }) \\
+33.08(\text { Temperature }+- \\
0.684(\text { Salinity })+- \\
1.257(\text { Depth:Temperature })+- \\
894.16\end{array}$ \\
\hline $\begin{array}{l}\text { QT } \\
\text { spp. }\end{array}$ & Quantile & Medium & & & & & & $\begin{array}{l}\text { Species Count } \sim-4.51 \text { (Depth) } \\
+6.21(\text { Temperature })+- \\
24.56(\mathrm{RDO})+ \\
0.75(\text { Depth:RDO })+30.57\end{array}$ \\
\hline $\begin{array}{l}\text { QT } \\
\text { spp. }\end{array}$ & Poisson & Medium & & & & & & $\begin{array}{l}\text { Species Count } \sim-4.84(\text { Depth }) \\
+-3.17(\text { Temperature }+ \\
0.56(\mathrm{RDO})+0.19(\text { Depth: } \\
\text { Temperature })+- \\
0.08(\text { Depth:RDO })+2.36(\mathrm{pH}) \\
+69.05\end{array}$ \\
\hline $\begin{array}{l}\text { QT } \\
\text { spp. }\end{array}$ & Linear & Medium & & & & & & $\begin{array}{l}\text { Species Count } \sim- \\
\text { 250.87(Depth) +- } \\
164.87(\text { Temperature })+ \\
32.32(\mathrm{RDO})+125.13(\mathrm{pH})+ \\
\text { 9.99(Depth:Temperature })+- \\
\text { 4.22(Depth:RDO) + 3403.62 }\end{array}$ \\
\hline $\begin{array}{l}\text { QT } \\
\text { spp. }\end{array}$ & Quantile & Fine & & & & & & $\begin{array}{l}\text { Species Count } \sim 0.57(\text { Depth })+ \\
-3.85(\mathrm{RDO})+66.65(\mathrm{pH})+- \\
482.25\end{array}$ \\
\hline $\begin{array}{l}\text { QT } \\
\text { spp. }\end{array}$ & Poisson & Fine & & & & & & $\begin{array}{l}\text { Species Count } \sim 0.01(\text { Depth })+ \\
3.51\end{array}$ \\
\hline $\begin{array}{l}\text { QT } \\
\text { spp. }\end{array}$ & Linear & Fine & & & & & & $\begin{array}{l}\text { Species Count } \sim 0.51(\text { Depth })+ \\
-3.71(\mathrm{RDO})+65.04(\mathrm{pH})+- \\
469.40\end{array}$ \\
\hline $\begin{array}{l}\text { QT } \\
\text { spp. }\end{array}$ & Quantile & Very Fine & & & & & & $\begin{array}{l}\text { Species Count } \sim-28.76(\text { Depth }) \\
+-17.99(\text { Temperature })+- \\
9.80(\text { RDO })+71.00(\mathrm{pH})+ \\
1.01(\text { Depth:Temperature })+ \\
22.29\end{array}$ \\
\hline $\begin{array}{l}\text { QT } \\
\text { spp. }\end{array}$ & Poisson & Very Fine & & & & & & Species Count $\sim 3.23$ \\
\hline $\begin{array}{l}\text { QT } \\
\text { spp. }\end{array}$ & Linear & Very Fine & & & & & & $\begin{array}{l}\text { Species Count -69.56(Depth) } \\
+-40.53(\text { Temperature })+- \\
9.51(\text { RDO })+ \\
2.47(\text { Depth:Temperature })+ \\
1242.89\end{array}$ \\
\hline
\end{tabular}


Table 3. Predictive Models of Homotrema rubrum fragment counts vs environmental parameters under each fragment-size interval, including color-coded inclusion-exclusion and formula.

\begin{tabular}{|c|c|c|c|c|c|c|c|c|}
\hline Taxa & Regression & $\begin{array}{l}\text { Fragment- } \\
\text { size Interval }\end{array}$ & Depth & Temperature & Salinity & RDO & pH & Formula \\
\hline $\mathrm{Hr}$ & Quantile & Coarse & & & & & & $\begin{array}{l}\text { Dead Fragment Count - } \\
3.95(\text { Depth })+-15.86 \text { (Temperature) } \\
+5.98(\text { RDO })+-0.98 \text { (Salinity) }+ \\
458.31\end{array}$ \\
\hline $\mathrm{Hr}$ & Poisson & Coarse & & & & & & $\begin{array}{l}\text { Dead Fragment Count } \sim \\
0.07 \text { (Depth) }+-0.30(\text { Temperature) } \\
+0.17(\text { RDO })+-0.02 \text { (Salinity) }+ \\
11.21\end{array}$ \\
\hline $\mathrm{Hr}$ & Linear & Coarse & & & & & & $\begin{array}{l}\text { Dead Fragment Count - } \\
32.40(\text { Depth })+- \\
36.53 \text { (Temperature })+21.62(\text { RDO }) \\
+-0.97 \text { (Salinity) }+ \\
1.27(\text { Depth:Temp })+- \\
0.997 \text { (Depth:RDO }+922.18\end{array}$ \\
\hline $\mathrm{Hr}$ & Quantile & Medium & & & & & & $\begin{array}{l}\text { Dead Fragment Count - } \\
5.39 \text { (Depth) }+-14.47 \text { (Temperature) } \\
+16.42(\mathrm{RDO})+1.07 \text { (Salinity) + } \\
180.079(\mathrm{pH})+1832.43\end{array}$ \\
\hline Hr. & Poisson & Medium & & & & & & $\begin{array}{l}\text { Dead Fragment Count } \\
6.72(\text { Depth })+4.31(\text { Temperature })+ \\
-0.57(\mathrm{RDO})+0.01(\text { Salinity })+- \\
6.93(\mathrm{pH})+-0.27(\text { Depth:Temp })+ \\
0.11(\text { Depth:RDO })+-56.21 \\
\end{array}$ \\
\hline $\mathrm{Hr}$ & Linear & Medium & & & & & & $\begin{array}{l}\text { Dead Fragment Count } ~ \\
164.2(\text { Depth })+ \\
101.7(\text { Temperature })+-6.56(\mathrm{RDO}) \\
+0.21(\text { Salinity })+-182.4(\mathrm{pH})+- \\
6.545(\text { Depth:Temp })+- \\
2.531 \text { (Depth:RDO) }+922.18\end{array}$ \\
\hline $\mathrm{Hr}$ & Quantile & Fine & & & & & & $\begin{array}{l}\text { Dead Fragment Count - } \\
1.62 \text { (Depth) }+-2.24 \text { (Temperature) } \\
+9.28(\mathrm{RDO})+0.30 \text { (Salinity) }+- \\
139.94(\mathrm{pH})+1183.13\end{array}$ \\
\hline $\mathrm{Hr}$ & Poisson & Fine & & & & & & $\begin{array}{l}\text { Dead Fragment Count - } \\
0.02(\text { Depth })+0.197(\text { RDO })+- \\
3.31(\mathrm{pH})+29.35\end{array}$ \\
\hline $\mathrm{Hr}$ & Linear & Fine & & & & & & $\begin{array}{l}\text { Dead Fragment Count } ~ \\
77.99(\text { Depth })+ \\
41.98(\text { Temperature })+14.34(\mathrm{RDO}) \\
+0.48(\text { Salinity })+-74.14(\mathrm{pH})+- \\
2.84(\text { Depth:Temp })+-652.26 \\
\end{array}$ \\
\hline $\mathrm{Hr}$ & Quantile & Very Fine & & & & & & $\begin{array}{l}\text { Dead Fragment Count } ~ \\
106.20(\text { Depth })+ \\
62.32 \text { (Temperature) }+14.18(\mathrm{RDO}) \\
+-3.76(\text { Depth:Temp })+-1836.75\end{array}$ \\
\hline $\mathrm{Hr}$ & Poisson & Very Fine & & & & & & $\begin{array}{l}\text { Dead Fragment Count } \sim \\
2.78(\text { Depth })+1.63 \text { Temperature })+ \\
0.34(\text { RDO })+-0.10(\text { Depth:Temp })+ \\
-45.02\end{array}$ \\
\hline $\mathrm{Hr}$ & Linear & Very Fine & & & & & & $\begin{array}{l}\text { Dead Fragment Count } \\
117.58 \text { (Depth) + } \\
57.44(\text { Temperature })+32.35(\mathrm{RDO}) \\
+0.55 \text { (Salinity) }+72.71(\mathrm{pH})+- \\
3.93(\text { Depth:Temp })+- \\
1.19 \text { (Depth:RDO) +-2442.06 }\end{array}$ \\
\hline
\end{tabular}


Table 4. Predictive Models of Elphidium spp. test counts vs environmental parameters under each fragmentsize interval, including color-coded inclusion-exclusion and formula.

\begin{tabular}{|c|c|c|c|c|c|c|c|c|}
\hline Taxa & Regression & $\begin{array}{l}\text { Test-size } \\
\text { Interval }\end{array}$ & Depth & Temperature & Salinity & RDO & pH & Formula \\
\hline $\mathrm{E}$ & Quantile & Medium & & & & & & $\begin{array}{l}\text { Species Count } \sim 0.55 \text { (Depth) }+ \\
-0.02\left(\text { Depth }^{2}\right)+2.53\end{array}$ \\
\hline $\mathrm{E}$ & Poisson & Medium & & & & & & $\begin{array}{l}\text { Species Count } \sim 0.12 \text { (Depth) }+ \\
-0.005\left(\text { Depth }^{2}\right)+1.15\end{array}$ \\
\hline $\mathrm{E}$ & Linear & Medium & & & & & & $\begin{array}{l}\text { Species Count } \sim 0.73 \text { (Depth) }+ \\
-0.05\left(\text { Depth }^{2}\right)+-1.22(\text { Temp })+ \\
0.15(\text { Salinity })+36.24\end{array}$ \\
\hline $\mathrm{E}$ & Quantile & Fine & & & & & & $\begin{array}{l}\text { Species Count } ~ 158.39 \text { (Depth) } \\
+85.58(\text { Temp })+0.43 \text { (Salinity) } \\
+280.19(\mathrm{pH})+- \\
\text { 5.67(Depth:Temp) }+-4723.78\end{array}$ \\
\hline $\mathrm{E}$ & Poisson & Fine & & & & & & $\begin{array}{l}\text { Species Count 29.832(Depth) } \\
+16.28(\text { Temp })+0.07 \text { (Salinity) } \\
+43.96(\mathrm{pH})+- \\
\text { 1.07(Depth:Temp) +-826.72 }\end{array}$ \\
\hline $\mathrm{E}$ & Linear & Fine & & & & & & $\begin{array}{l}\text { Species Count 200.10(Depth) } \\
+108.80(\text { Temp })+2.78(\mathrm{RDO}) \\
+0.52(\text { Salinity })+317.53(\mathrm{pH}) \\
+-7.15(\text { Depth:Temp })+- \\
5711.50\end{array}$ \\
\hline $\mathrm{E}$ & Quantile & Very Fine & & & & & & $\begin{array}{l}\text { Species Count } ~ 133.44 \text { (Depth) } \\
+71.45(\text { Temp })+0.30 \text { (Salinity) } \\
+235.73(\mathrm{pH})+- \\
4.75(\text { Depth:Temp) }+-3961.46\end{array}$ \\
\hline $\mathrm{E}$ & Poisson & Very Fine & & & & & & $\begin{array}{l}\text { Species Count } ~ 25.05(\text { Depth }) \\
+13.72(\text { Temp })+0.87(\mathrm{RDO})+ \\
0.05(\text { Salinity })+33.21(\mathrm{pH})+- \\
0.89(\text { Depth:Temp })+-667.37 \\
\end{array}$ \\
\hline $\mathrm{E}$ & Linear & Very Fine & & & & & & $\begin{array}{l}\text { Species Count 195.76(Depth) } \\
+106.53(\text { Temp })+4.54(\mathrm{RDO}) \\
+0.46(\text { Salinity })+285.43(\mathrm{pH}) \\
+-6.98(\text { Depth:Temp })+- \\
5399.57\end{array}$ \\
\hline
\end{tabular}


Table 5. Predictive Models of Ammonia beccarii test counts vs environmental parameters under each fragment-size interval, including color-coded inclusion-exclusion and formula.

\begin{tabular}{|c|c|c|c|c|c|c|c|c|}
\hline Taxa & Regression & $\begin{array}{l}\text { Test-size } \\
\text { Interval }\end{array}$ & Depth & Temperature & Salinity & RDO & pH & Formula \\
\hline $\mathrm{Ab}$ & Quantile & Medium & & & & & & $\begin{array}{l}\text { Species Count } \sim 0.83 \text { (Depth) }+ \\
-0.03\left(\text { Depth }^{2}\right)+1.80\end{array}$ \\
\hline $\mathrm{Ab}$ & Poisson & Medium & & & & & & $\begin{array}{l}\text { Species Count } \sim 9.36 \text { (Depth) }+ \\
5.29(\text { Temp })+17.15(\mathrm{pH})+- \\
0.33 \text { (Depth:Temp) }+-289.20\end{array}$ \\
\hline $\mathrm{Ab}$ & Linear & Medium & & & & & & $\begin{array}{l}\text { Species Count } ~ 110.00(\text { Depth }) \\
+59.79(\text { Temp })+3.308(\mathrm{RDO}) \\
+30.17(\text { Salinity })+138.20(\mathrm{pH}) \\
+-408.10(\text { Depth } \\
\text { 2) }+- \\
\text { 3.90(Depth:Temp }+-2855.00\end{array}$ \\
\hline $\mathrm{Ab}$ & Quantile & Fine & & & & & & $\begin{array}{l}\text { Species Count .33(Depth) }+ \\
13.00\end{array}$ \\
\hline $\mathrm{Ab}$ & Poisson & Fine & & & & & & $\begin{array}{l}\text { Species Count } \sim 0.02 \text { (Depth) }+ \\
2.62\end{array}$ \\
\hline $\mathrm{Ab}$ & Linear & Fine & & & & & & $\begin{array}{l}\text { Species Count - } \\
106.46(\text { Depth })+-57.06 \text { (Temp) } \\
+-3.09(\mathrm{RDO})+-0.43 \text { (Salinity) } \\
+-144.22(\mathrm{pH})+ \\
\text { 3.83(Depth:Temp) + 2845.08 }\end{array}$ \\
\hline$\overline{\mathrm{Ab}}$ & Quantile & Very Fine & & & & & & $\begin{array}{l}\text { Species Count } \sim-0.38 \text { (Depth) } \\
+3.49\end{array}$ \\
\hline $\mathrm{Ab}$ & Poisson & Very Fine & & & & & & $\begin{array}{l}\text { Species Count } \sim-0.01 \text { (Depth) } \\
+3.49\end{array}$ \\
\hline$\overline{A b}$ & Linear & Very Fine & & & & & & $\begin{array}{l}\text { Species Count - } \\
182.04(\text { Depth }+-98.40 \text { (Temp) } \\
+-8.02(\mathrm{RDO})+-0.64 \text { (Salinity) } \\
+-204.21(\mathrm{pH})+ \\
6.51(\text { Depth:Temp }+4569.37\end{array}$ \\
\hline
\end{tabular}


Table 6. Global Hypothesis ANOVA tests of coarse test/fragment-size fraction of QT | Hr | E | Ab

Environmental factors. $H_{0}$ : All factor levels have same effect on dead test and fragment counts; $H_{a}$ : At least one factor level has different effect on dead test and fragment counts. (A) Depth; (B) Temperature; (C)

A

Salinity; (D) RDO; (E) pH.

\begin{tabular}{|c|c|c|c|c|c|c|c|}
\hline $\begin{array}{l}\text { Test } \\
\text { Type: } \\
\text { Depth }\end{array}$ & $\begin{array}{l}\text { Test } \\
\text { Statistic }\end{array}$ & df1 & df2 & $\begin{array}{l}\text { p- } \\
\text { value }\end{array}$ & $\begin{array}{l}\text { Permutation p- } \\
\text { value }\end{array}$ & $\begin{array}{l}\text { Global } \\
\text { Hypothesis } \\
\text { significant? }\end{array}$ & $\begin{array}{l}\text { Subset } \\
\text { Algorithm } \\
\text { continue? }\end{array}$ \\
\hline ANOVA & 26.427 & 3.646 & 12.5148 & 0.00 & 0.00 & \multirow[t]{4}{*}{ Yes } & \multirow[t]{4}{*}{ Yes } \\
\hline LH Test & NA & NA & NA & NA & NA & & \\
\hline BNP Test & NA & NA & NA & NA & NA & & \\
\hline $\begin{array}{l}\text { Wilks } \\
\text { Lambda }\end{array}$ & NA & NA & NA & NA & $\begin{array}{l}\text { NA, rank is } \\
\text { singular }\end{array}$ & & \\
\hline
\end{tabular}

B

\begin{tabular}{|l|l|l|l|l|l|l|c|}
\hline $\begin{array}{l}\text { Test Type: } \\
\text { Temperature }\end{array}$ & $\begin{array}{l}\text { Test } \\
\text { Statistic }\end{array}$ & df1 & df2 & $\begin{array}{l}\text { p- } \\
\text { value }\end{array}$ & $\begin{array}{l}\text { Permutation p- } \\
\text { value }\end{array}$ & $\begin{array}{l}\text { Global } \\
\text { Hypothesis } \\
\text { significant? }\end{array}$ & $\begin{array}{l}\text { Subset } \\
\text { Algorithm } \\
\text { continue? }\end{array}$ \\
\hline ANOVA & 5.554 & 2.23 & 7.0467 & 0.034 & 0.068 & Yes & Yes \\
\hline LH Test & NA & NA & NA & NA & NA & \\
\hline BNP Test & NA & NA & NA & NA & NA & \\
\cline { 1 - 1 } & NA & NA & NA & NA & $\begin{array}{l}\text { NA, rank is } \\
\text { singular }\end{array}$ \\
Lambda & & & & & & \\
\hline
\end{tabular}

C

\begin{tabular}{|c|c|c|c|c|c|c|c|}
\hline $\begin{array}{l}\text { Test } \\
\text { Type: } \\
\text { Salinity }\end{array}$ & $\begin{array}{l}\text { Test } \\
\text { Statistic }\end{array}$ & df1 & df2 & $\begin{array}{l}\text { p- } \\
\text { value }\end{array}$ & $\begin{array}{l}\text { Permutation p- } \\
\text { value }\end{array}$ & $\begin{array}{l}\text { Global } \\
\text { Hypothesis } \\
\text { significant? }\end{array}$ & $\begin{array}{l}\text { Subset } \\
\text { Algorithm } \\
\text { continue? }\end{array}$ \\
\hline ANOVA & 7.383 & 2.621 & 15.1626 & 0.004 & 0.011 & \multirow[t]{4}{*}{ Yes } & \multirow[t]{4}{*}{ Yes } \\
\hline LH Test & NA & NA & NA & NA & NA & & \\
\hline BNP Test & NA & NA & NA & NA & NA & & \\
\hline $\begin{array}{l}\text { Wilks } \\
\text { Lambda }\end{array}$ & NA & NA & NA & NA & $\begin{array}{l}\text { NA, rank is } \\
\text { singular }\end{array}$ & & \\
\hline
\end{tabular}

D

\begin{tabular}{|c|c|c|c|c|c|c|c|}
\hline $\begin{array}{l}\text { Test } \\
\text { Type: } \\
\text { RDO }\end{array}$ & $\begin{array}{l}\text { Test } \\
\text { Statistic }\end{array}$ & df1 & df2 & $\begin{array}{l}\text { p- } \\
\text { value }\end{array}$ & $\begin{array}{l}\text { Permutation p- } \\
\text { value }\end{array}$ & $\begin{array}{l}\text { Global } \\
\text { Hypothesis } \\
\text { significant? }\end{array}$ & $\begin{array}{l}\text { Subset } \\
\text { Algorithm } \\
\text { continue? }\end{array}$ \\
\hline ANOVA & 17.696 & 4.382 & 9.975 & 0.00 & 0.001 & \multirow[t]{4}{*}{ Yes } & \multirow[t]{4}{*}{ Yes } \\
\hline LH Test & NA & NA & NA & NA & NA & & \\
\hline BNP Test & NA & NA & NA & NA & NA & & \\
\hline $\begin{array}{l}\text { Wilks } \\
\text { Lambda }\end{array}$ & NA & NA & $\mathrm{NA}$ & NA & $\begin{array}{l}\text { NA, rank is } \\
\text { singular }\end{array}$ & & \\
\hline
\end{tabular}

\section{E}

\begin{tabular}{|c|c|c|c|c|c|c|c|}
\hline $\begin{array}{l}\text { Test } \\
\text { Type: pH }\end{array}$ & $\begin{array}{l}\text { Test } \\
\text { Statistic }\end{array}$ & df1 & df2 & $\begin{array}{l}\text { p- } \\
\text { value }\end{array}$ & $\begin{array}{l}\text { Permutation p- } \\
\text { value }\end{array}$ & $\begin{array}{l}\text { Global } \\
\text { Hypothesis } \\
\text { significant? }\end{array}$ & $\begin{array}{l}\text { Subset } \\
\text { Algorithm } \\
\text { continue? }\end{array}$ \\
\hline ANOVA & 10.749 & 2.543 & 8.728 & 0.03 & 0.011 & \multirow[t]{4}{*}{ Yes } & \multirow[t]{4}{*}{ Yes } \\
\hline LH Test & NA & NA & NA & NA & NA & & \\
\hline BNP Test & NA & NA & $\mathrm{NA}$ & NA & NA & & \\
\hline $\begin{array}{l}\text { Wilks } \\
\text { Lambda }\end{array}$ & NA & NA & NA & NA & $\begin{array}{l}\text { NA, rank is } \\
\text { singular }\end{array}$ & & \\
\hline
\end{tabular}


Table 7. Subset Algorithm on test/fragment counts of coarse size-fraction of QT | Hr | E | Ab. $H_{0}$ : Counts for all taxa are equal; $\mathrm{H}_{\mathrm{a}}$ : At least one taxa group is not equal.

\begin{tabular}{|l|l|l|l|l|l|l|}
\hline $\begin{array}{l}\text { Environmental } \\
\text { Parameter }\end{array}$ & QT & Hr & E & Ab & Statistical Exceptions & Color key \\
\hline Depth & & & & & & *At least one taxa is not equal \\
\hline Temperature & & & & & Most statistically similar taxa \\
\hline Salinity & & & & & & $\begin{array}{l}\text { 1st order statistically } \\
\text { distinguishable taxa }\end{array}$ \\
\hline RDO & & & & & $\begin{array}{l}2^{\text {nd }} \text { order statistically } \\
\text { distinguishable taxa }\end{array}$ \\
\hline pH & & & & & & \\
\hline
\end{tabular}


Table 8. Subset Algorithm on depth zones of coarse size-fraction of QT $|\mathrm{Hr}| \mathrm{E} \mid \mathrm{Ab}$. $\mathrm{H}_{\mathbf{0}}$ : All depth zones contributed equally to significant differences in counts. $\mathrm{H}_{\mathrm{a}}$ : At least one depth zone contributed differently to significant differences in counts

\begin{tabular}{|l|l|}
\hline Depth Zones & Color Key \\
\hline Shallow Sandy Zone $(0-10 \mathrm{~m})$ & $\begin{array}{l}\text { 1st order statistically } \\
\text { distinguishable zone }\end{array}$ \\
\hline Oculina Zone $(10-18 \mathrm{~m})$ & $\begin{array}{l}\mathbf{3}^{\text {rd }} \text { order statistically } \\
\text { distinguishable zone }\end{array}$ \\
\hline Subthermocline Zone $(18-25 \mathrm{~m})$ & $\begin{array}{l}\mathbf{2}^{\text {nd }} \text { order statistically } \\
\text { distinguishable zone }\end{array}$ \\
\hline
\end{tabular}


Table 9. Subset Algorithm on temperature zones of coarse size-fraction of QT | Hr | E | Ab. $\mathrm{H}_{0}$ : All temperature zones contributed equally to significant differences in counts. $\mathrm{H}_{\mathrm{a}}$ : At least one temperature zone contributed differently to significant differences in counts.

\begin{tabular}{|l|l|}
\hline Temperature Zones & Color Key \\
\hline Mixed Layer (26-28 C) & $\begin{array}{l}\mathbf{1}^{\text {st }} \text { order statistically } \\
\text { distinguishable zone }\end{array}$ \\
\hline Thermocline Layer (24-26 C) & Most statistically similar zone \\
\hline Subthermocline Layer (22-24 C) & Most statistically similar zone \\
\hline
\end{tabular}


Table 10. Subset Algorithm on salinity zones of coarse size-fraction of QT | Hr | E | Ab. $\mathrm{H}_{0}$ : All salinity level zones contributed equally to significant differences in counts; $H_{a}$ : At least salinity level zone contributed differently to significant differences in counts.

\begin{tabular}{|l|l|}
\hline Salinity Zones & Color Key \\
\hline Mesohaline (2-10 psu) & $\begin{array}{l}\mathbf{1}^{\text {st }} \text { order statistically } \\
\text { distinguishable zone }\end{array}$ \\
\hline Polyhaline (10-35 psu) & Most statistically similar zone \\
\hline Euhaline (35+ psu) & Most statistically similar zone \\
\hline
\end{tabular}


Table 11. Subset Algorithm on dissolved oxygen zones of coarse size-fraction of QT | Hr | E | Ab. $\mathbf{H}_{0}$ : All dissolved oxygen level zones contributed equally to significant differences in counts; $\mathrm{H}_{\mathrm{a}}$ : At least one dissolved oxygen level zone contributed differently to significant differences in counts

\begin{tabular}{|l|l|}
\hline RDO Zones & Color Key \\
\hline $0-2 \mathrm{mg} / \mathrm{L}$ & Most statistically similar zone \\
\hline $2-4 \mathrm{mg} / \mathrm{L}$ & Most statistically similar zone \\
\hline $4-6 \mathrm{mg} / \mathrm{L}$ & $\mathbf{2}^{\text {nd }}$ order statistically similar zone \\
\hline $6-9 \mathrm{mg} / \mathrm{L}$ & $\mathbf{2}^{\text {nd }}$ order statistically similar zone \\
\hline
\end{tabular}


Table 12. Subset Algorithm on $\mathbf{p H}$ zones coarse size-fraction of QT $|\mathrm{Hr}| \mathrm{E} \mid \mathrm{Ab}$. $\mathrm{H}_{\mathrm{o}}$ : All pH intervals contributed equally to significant differences in counts; $\mathrm{H}_{\mathrm{a}}$ : At least one $\mathrm{pH}$ interval contributed differently to significant differences in counts

\begin{tabular}{|l|l|}
\hline pH Zones & Color Key \\
\hline $8.0-8.2$ & Most statistically similar zone \\
\hline $7.8-8.0$ & Most statistically similar zone \\
\hline $7.6-7.8$ & $\begin{array}{l}\mathbf{1}^{\text {st }} \text { order statistically } \\
\text { distinguishable zone }\end{array}$ \\
\hline
\end{tabular}


Table 13. Relative probabilities results on environmental factors of coarse size-fraction of QT $|\mathrm{Hr}| \mathrm{E} \mid \mathrm{Ab}$. (A) Depth; (B) Temperature; (C) Salinity; (D) RDO; (E) pH.

A

\begin{tabular}{|c|c|c|c|}
\hline 1/2+ mm QT spp. & 1/2+ mm Hr & 1/2+ mm E spp. & 1/2+ mm Ab \\
\hline $\begin{array}{l}0.944, \\
\text { Subthermocline Zone }\end{array}$ & $\begin{array}{l}\text { 0.759, Shallow Sandy } \\
\text { Zone }\end{array}$ & $\begin{array}{l}\text { 0.722, Shallow Sandy } \\
\text { Zone }\end{array}$ & 0.500 , any depth zone \\
\hline
\end{tabular}

B

\begin{tabular}{|l|l|l|l|}
\hline $\mathbf{1 / 2 +} \mathbf{m m}$ QT spp. & $\mathbf{1 / 2 +} \mathbf{~ m m ~ H r}$ & $\mathbf{1 / 2 +} \mathbf{~ m m}$ E spp. & $\mathbf{1 / 2 +} \mathbf{~ m m ~ A b}$ \\
\hline $\begin{array}{l}\text { 0.944, Subthermocline } \\
\text { Layer }\end{array}$ & $\begin{array}{l}\text { 0.574, Mixed Layer } \\
\text { 0.556, Mixed Layer }\end{array}$ & $\begin{array}{l}\text { 0.500, any temperature } \\
\text { zone }\end{array}$ \\
\hline
\end{tabular}

\begin{tabular}{|c|c|c|c|}
\hline 1/2+ mm QT spp. & $1 / 2+\mathrm{mm} \mathrm{Hr}$ & 1/2+ mm E spp. & 1/2+ mm Ab \\
\hline 0.944, Euhaline Zone & $\begin{array}{l}\text { 0.656, Mesohaline } \\
\text { Zone }\end{array}$ & 0.589, Mesohaline Zone & 0.500 , any salinity zone \\
\hline
\end{tabular}

D

\begin{tabular}{|l|l|l|l|}
\hline 1/2+ mm QT spp. & $\mathbf{1 / 2 +} \mathbf{~ m m ~ H r}$ & $\mathbf{1} / \mathbf{2 +} \mathbf{~ m m}$ E $\mathbf{~ s p p}$. & $\mathbf{1 / 2 +} \mathbf{~ m m ~ A b}$ \\
\hline $0.944,0-2 \mathrm{mg} / \mathrm{L} ; 0.833,2-4 \mathrm{mg} / \mathrm{L}$ & $0.694,6-9 \mathrm{mg} / \mathrm{L}$ & $0.639,6-9 \mathrm{mg} / \mathrm{L}$ & 0.500, any RDO zone \\
\hline
\end{tabular}
E

\begin{tabular}{|c|c|c|c|}
\hline 1/2+ mm QT spp. & 1/2+ mm Hr & 1/2+ mm E spp. & 1/2+ mm Ab \\
\hline $0.944,7.6-7.8 ; 0.546,7.8-8.0$ & $0.617,8.0-8.2$ & $0.589,8.0-8.2$ & 0.500, any $\mathrm{pH}$ zone \\
\hline
\end{tabular}


Table 14. Global Hypothesis ANOVA tests of medium test/fragment-size fraction of QT | Hr | E | Ab

Environmental factors. $H_{0}$ : All factor levels have same effect on dead test and fragment counts; $H_{a}$ : At least one factor level has different effect on dead test and fragment counts. (A) Depth; (B) Temperature; (C)

A

Salinity; (D) RDO; (E) pH.

\begin{tabular}{|c|c|c|c|c|c|c|c|}
\hline Test Type & $\begin{array}{l}\text { Test } \\
\text { Statistic }\end{array}$ & df1 & df 2 & $\begin{array}{l}\text { p- } \\
\text { value }\end{array}$ & $\begin{array}{l}\text { Permutation p- } \\
\text { value }\end{array}$ & $\begin{array}{l}\text { Global } \\
\text { Hypothesis } \\
\text { significant? }\end{array}$ & $\begin{array}{l}\text { Subset } \\
\text { Algorithm } \\
\text { continue? }\end{array}$ \\
\hline ANOVA & 17.587 & 4.314 & 14.8069 & 0.000 & 0.000 & \multirow[t]{4}{*}{ Yes } & \multirow[t]{4}{*}{ Yes } \\
\hline LH Test & 7.245 & 8.000 & 15.000 & 0.001 & 0.000 & & \\
\hline BNP Test & 5.127 & 9.434 & 28.9577 & 0.000 & 0.000 & & \\
\hline $\begin{array}{l}\text { Wilks } \\
\text { Lambda }\end{array}$ & 6.186 & 8.000 & 24.000 & 0.000 & 0.000 & & \\
\hline
\end{tabular}

B

\begin{tabular}{|c|c|c|c|c|c|c|c|}
\hline Test Type & $\begin{array}{l}\text { Test } \\
\text { Statistic }\end{array}$ & df1 & df2 & $\begin{array}{l}\text { p- } \\
\text { value }\end{array}$ & $\begin{array}{l}\text { Permutation p- } \\
\text { value }\end{array}$ & $\begin{array}{l}\text { Global } \\
\text { Hypothesis } \\
\text { significant? }\end{array}$ & $\begin{array}{l}\text { Subset } \\
\text { Algorithm } \\
\text { continue? }\end{array}$ \\
\hline ANOVA & 8.561 & 4.585 & 14.4861 & 0.001 & 0.003 & \multirow[t]{4}{*}{ Yes } & \multirow[t]{4}{*}{ Yes } \\
\hline LH Test & 2.2802 & 8.000 & 15.000 & 0.041 & 0.045 & & \\
\hline BNP Test & 1.559 & 9.434 & 28.9577 & 0.172 & 0.147 & & \\
\hline $\begin{array}{l}\text { Wilks } \\
\text { Lambda }\end{array}$ & 2.172 & 8.000 & 24.000 & 0.068 & $\begin{array}{l}0.071, \text { rank is } \\
\text { singular }\end{array}$ & & \\
\hline
\end{tabular}

C

\begin{tabular}{|l|l|l|l|l|l|l|c|}
\hline Test Type & $\begin{array}{l}\text { Test } \\
\text { Statistic }\end{array}$ & df1 & df2 & $\begin{array}{l}\text { p- } \\
\text { value }\end{array}$ & $\begin{array}{l}\text { Permutation p- } \\
\text { value }\end{array}$ & $\begin{array}{l}\text { Global } \\
\text { Hypothesis } \\
\text { significant? }\end{array}$ & $\begin{array}{l}\text { Subset } \\
\text { Algorithm } \\
\text { continue? }\end{array}$ \\
\hline ANOVA & 7.187 & 3.875 & 22.4220 & 0.001 & 0.000 & Yes & Yes \\
\hline LH Test & 4.496 & 8.000 & 15.000 & 0.006 & 0.005 & \\
\hline BNP Test & 4.594 & 9.434 & 28.9577 & 0.001 & 0.000 & & \\
\cline { 1 - 1 } $\begin{array}{l}\text { Wilks } \\
\text { Lambda }\end{array}$ & 4.582 & 8.000 & 24.000 & 0.002 & 0.002 & & \\
\hline
\end{tabular}

D

\begin{tabular}{|c|c|c|c|c|c|c|c|}
\hline Test Type & $\begin{array}{l}\text { Test } \\
\text { Statistic }\end{array}$ & df1 & df2 & $\begin{array}{l}\text { p- } \\
\text { value }\end{array}$ & $\begin{array}{l}\text { Permutation p- } \\
\text { value }\end{array}$ & $\begin{array}{l}\text { Global } \\
\text { Hypothesis } \\
\text { significant? }\end{array}$ & $\begin{array}{l}\text { Subset } \\
\text { Algorithm } \\
\text { continue? }\end{array}$ \\
\hline ANOVA & 11.158 & 5.445 & 12.3955 & 0.000 & 0.000 & \multirow[t]{4}{*}{ Yes } & \multirow[t]{4}{*}{ Yes } \\
\hline LH Test & 9.173 & 12.000 & 15.3963 & 0.000 & 0.000 & & \\
\hline BNP Test & 2.433 & 15.448 & 47.4161 & 0.01 & 0.006 & & \\
\hline $\begin{array}{l}\text { Wilks } \\
\text { Lambda }\end{array}$ & 4.904 & 12.000 & 29.3948 & 0.000 & 0.000 & & \\
\hline
\end{tabular}

E

\begin{tabular}{|c|c|c|c|c|c|c|c|}
\hline Test Type & $\begin{array}{l}\text { Test } \\
\text { Statistic }\end{array}$ & df1 & df 2 & $\begin{array}{l}\text { p- } \\
\text { value }\end{array}$ & $\begin{array}{l}\text { Permutation p- } \\
\text { value }\end{array}$ & $\begin{array}{l}\text { Global } \\
\text { Hypothesis } \\
\text { significant? }\end{array}$ & $\begin{array}{l}\text { Subset } \\
\text { Algorithm } \\
\text { continue? }\end{array}$ \\
\hline ANOVA & 9.658 & 4.095 & 14.0552 & 0.001 & 0.001 & \multirow[t]{4}{*}{ Yes } & \multirow[t]{4}{*}{ Yes } \\
\hline LH Test & 4.349 & 8.000 & 15.000 & 0.007 & 0.012 & & \\
\hline BNP Test & 2.190 & 9.434 & 28.9577 & 0.051 & 0.042 & & \\
\hline $\begin{array}{l}\text { Wilks } \\
\text { Lambda }\end{array}$ & 3.219 & 8.000 & 24.000 & 0.012 & 0.019 & & \\
\hline
\end{tabular}


Table 15. Subset Algorithm on test/fragment counts of medium size-fraction of QT | Hr | E | Ab. $\mathrm{H}_{0}$ : Counts for all taxa are equal; $H_{a}$ : At least one taxa group is not equal.

\begin{tabular}{|l|l|l|l|l|l|l|}
\hline $\begin{array}{l}\text { Environmental } \\
\text { Parameter }\end{array}$ & QT & Hr & E & Ab & $\begin{array}{l}\text { Statistical } \\
\text { Exceptions }\end{array}$ & Color key \\
\hline Depth & & & & & & \\
\hline Temperature & & & & & & Most statistically similar taxa \\
\hline Salinity & & & & & & $\begin{array}{l}\text { 1st order statistically } \\
\text { distinguishable taxa }\end{array}$ \\
\hline RDO & & & & & & $\begin{array}{l}\mathbf{2}^{\text {nd }} \text { order statistically } \\
\text { distinguishable taxa }\end{array}$ \\
\hline pH & & & Indistinguishable* & & & \\
\hline
\end{tabular}


Table 16. Subset Algorithm on depth zones of medium size-fraction of QT | Hr | E | Ab. $\mathrm{H}_{0}$ : All depth zones contributed equally to significant differences in counts. $\mathrm{H}_{\mathrm{a}}$ : At least one depth zone contributed differently to significant differences in counts

\begin{tabular}{|l|l|}
\hline Depth Zones & Color Key \\
\hline Shallow Sandy Zone $(0-10 \mathrm{~m})$ & $\begin{array}{l}\text { 1st order statistically } \\
\text { distinguishable zone }\end{array}$ \\
\hline Oculina Zone $(10-18 \mathrm{~m})$ & Most statistically similar zone \\
\hline Subthermocline Zone $(18-25 \mathrm{~m})$ & Most statistically similar zone \\
\hline
\end{tabular}


Table 17. Subset Algorithm on temperature zones of medium size-fraction of QT | Hr | E | Ab. $\mathrm{H}_{0}$ : All temperature zones contributed equally to significant differences in counts. $\mathrm{H}_{\mathrm{a}}$ : At least one temperature zone contributed differently to significant differences in counts.

\begin{tabular}{|l|l|}
\hline Temperature Zones & Color Key \\
\hline Mixed Layer (26-28 C) & Most statistically similar zone \\
\hline Thermocline Layer (24-26 C) & Most statistically similar zone \\
\hline Subthermocline Layer (22-24 C) & $\begin{array}{l}\text { 1st order statistically } \\
\text { distinguishable zone }\end{array}$ \\
\hline
\end{tabular}


Table 18. Subset Algorithm on salinity zones of medium size-fraction of QT | Hr | E | Ab. $\mathrm{H}_{0}$ : All salinity level zones contributed equally to significant differences in counts; $H_{a}$ : At least salinity level zone contributed differently to significant differences in counts.

\begin{tabular}{|l|l|}
\hline Salinity Zones & Color Key \\
\hline Mesohaline (2-10 psu) & $\begin{array}{l}\text { 1st order statistically } \\
\text { distinguishable zone }\end{array}$ \\
\hline Polyhaline (10-35 psu) & Most statistically similar zone \\
\hline Euhaline (35+ psu) & Most statistically similar zone \\
\hline
\end{tabular}


Table 19. Subset Algorithm on dissolved oxygen zones of medium size-fraction of QT | Hr | E | Ab. $\mathrm{H}_{0}$ : All dissolved oxygen level zones contributed equally to significant differences in counts; $H_{a}$ : At least one dissolved oxygen level zone contributed differently to significant differences in counts.

\begin{tabular}{|l|l|}
\hline RDO Zones & Color Key \\
\hline $0-2 \mathrm{mg} / \mathrm{L}$ & Most statistically similar zone \\
\hline $2-4 \mathrm{mg} / \mathrm{L}$ & Most statistically similar zone \\
\hline $4-6 \mathrm{mg} / \mathrm{L}$ & $\begin{array}{l}\text { 1st order statistically } \\
\text { distinguishable zone }\end{array}$ \\
\hline $6-9 \mathrm{mg} / \mathrm{L}$ & $\begin{array}{l}\text { 2nd order statistically } \\
\text { distinguishable zone }\end{array}$ \\
\hline
\end{tabular}


Table 20. Subset Algorithm on pH zones of medium size-fraction of QT | Hr | E | Ab. All pH intervals contributed equally to significant differences in counts; $\mathrm{H}_{\mathrm{a}}$ : At least one $\mathrm{pH}$ interval contributed differently to significant differences in counts.

\begin{tabular}{|l|l|}
\hline pH Zones & Color Key \\
\hline $8.0-8.2$ & $\begin{array}{l}\text { 3rd order statistically } \\
\text { distinguishable zone }\end{array}$ \\
\hline $7.8-8.0$ & $\begin{array}{l}\text { 2nd order statistically } \\
\text { distinguishable zone }\end{array}$ \\
\hline $7.6-7.8$ & $\begin{array}{l}\text { 1st order statistically } \\
\text { distinguishable zone }\end{array}$ \\
\hline
\end{tabular}


Table 21. Relative probabilities results on environmental factors of medium size-fraction of QT | Hr | E | Ab. (A) Depth; (B) Temperature; (C) Salinity; (D) RDO; (E) pH.

A

\begin{tabular}{|l|l|l|l|}
\hline 1/4 - 1/2 mm QT spp. & $\mathbf{1 / 4}-\mathbf{1 / 2} \mathbf{~ m m ~ H r}$ & $\mathbf{1 / 4}-\mathbf{1 / 2} \mathbf{~ m m ~ E ~ s p p . ~}$ & $\mathbf{1 / 4}-\mathbf{1 / 2} \mathbf{~ m m ~ A b}$ \\
\hline $\begin{array}{l}\text { 0.889, Subthermocline } \\
\text { Zone; 0.622, Oculina } \\
\text { Zone }\end{array}$ & $\begin{array}{l}\text { 0.829, Shallow } \\
\text { Sandy Zone }\end{array}$ & 0.661, Oculina Zone & $\begin{array}{l}0.586, \text { Oculina } \\
\text { Zone }\end{array}$ \\
\hline
\end{tabular}

B

\begin{tabular}{|l|l|l|l|}
\hline $\mathbf{1 / 4}-\mathbf{1 / 2} \mathbf{~ m m}$ QT spp. & $\mathbf{1 / 4}-\mathbf{1 / 2} \mathbf{~ m m ~ H r}$ & $\mathbf{1 / 4}-\mathbf{1 / 2} \mathbf{~ m m ~ E ~ s p p . ~}$ & $\mathbf{1 / 4}-\mathbf{1 / 2} \mathbf{~ m m ~ A b}$ \\
\hline $\begin{array}{l}\text { 0.889, Subthermocline } \\
\begin{array}{l}\text { Layer; 0.556, } \\
\text { Thermocline Layer }\end{array}\end{array}$ & 0.565, Mixed Layer & 0.565, Mixed Layer & $\begin{array}{l}0.586, \text { Thermocline } \\
\text { Layer }\end{array}$ \\
\hline
\end{tabular}

C

\begin{tabular}{|l|l|l|l|}
\hline $\mathbf{1 / 4}-\mathbf{1 / 2} \mathbf{m m}$ QT spp. & $\mathbf{1 / 4}-\mathbf{1 / 2} \mathbf{~ m m ~ H r}$ & $\mathbf{1 / 4}-\mathbf{1 / 2} \mathbf{~ m m ~ E ~ s p p . ~}$ & $\mathbf{1 / 4}-\mathbf{1 / 2} \mathbf{~ m m ~ A b}$ \\
\hline 0.750, Euhaline Zone; & 0.686, Mesohaline & 0.833, Polyhaline & 0.840, Polyhaline \\
0.694, Polyhaline Zone & Zone & Zone & Zone \\
\hline
\end{tabular}

D

\begin{tabular}{|l|l|l|l|}
\hline 1/4 - 1/2 mm QT spp. & $\mathbf{1 / 4}-\mathbf{1 / 2} \mathbf{~ m m ~ H r}$ & $\mathbf{1 / 4}-\mathbf{1 / 2} \mathbf{~ m m ~ E ~} \mathbf{~ s p p .}$ & $\mathbf{1 / 4}-\mathbf{1 / 2} \mathbf{~ m m ~ A b}$ \\
\hline $0.889,0-2 \mathrm{mg} / \mathrm{L} ; 0.722$, & $0.747,6-9 \mathrm{mg} / \mathrm{L}$ & $0.699,4-6 \mathrm{mg} / \mathrm{L}$ & $0.587,6-9 \mathrm{mg} / \mathrm{L} ;$ \\
$\begin{array}{l}2-4 \mathrm{mg} / \mathrm{L} ; 0.630,4-6 \\
\mathrm{mg} / \mathrm{L}\end{array}$ & & & $0.551,4-6 \mathrm{mg} / \mathrm{L}$ \\
\hline
\end{tabular}

E

\begin{tabular}{|l|l|l|l|}
\hline 1/4 - 1/2 mm QT spp. & $\mathbf{1 / 4}-\mathbf{1 / 2} \mathbf{~ m m ~ H r}$ & $\mathbf{1 / 4}-\mathbf{1 / 2} \mathbf{~ m m ~ E ~ s p p . ~}$ & $\mathbf{1 / 4}-\mathbf{1 / 2} \mathbf{~ m m ~ A b}$ \\
\hline $0.889,7.6-7.8 ; 0.574$, & $0.608,8.0-8.2$ & $0.586,7.8-8.0 ; 0.533$, & $0.633,8.0-8.2$ \\
$7.8-8.0$ & & $8.0-8.2$ & \\
\hline
\end{tabular}


Table 22. Global Hypothesis ANOVA tests of fine test/fragment-size fraction of QT | Hr | E | Ab

Environmental factors. $H_{0}$ : All factor levels have same effect on dead test and fragment counts; $H_{a}$ : At least one factor level has different effect on dead test and fragment counts. (A) Depth; (B) Temperature; (C)

A

Salinity; (D) RDO; (E) pH

\begin{tabular}{|l|l|l|l|l|l|l|c|}
\hline Test Type & $\begin{array}{l}\text { Test } \\
\text { Statistic }\end{array}$ & df1 & $\mathbf{d f 2}$ & $\begin{array}{l}\text { p- } \\
\text { value }\end{array}$ & $\begin{array}{l}\text { Permutation p- } \\
\text { value }\end{array}$ & $\begin{array}{l}\text { Global } \\
\text { Hypothesis } \\
\text { significant? }\end{array}$ & $\begin{array}{l}\text { Subset } \\
\text { Algorithm } \\
\text { continue? }\end{array}$ \\
\hline ANOVA & 9.995 & 3.962 & 13.5975 & 0.001 & 0.001 & Yes & Yes \\
\hline LH Test & 6.764 & 8.000 & 15.000 & 0.001 & 0.001 & \\
\cline { 1 - 2 } & 2.690 & 9.434 & 28.9577 & 0.020 & 0.012 & \\
\cline { 1 - 4 } $\begin{array}{l}\text { Wilks } \\
\text { Lambda }\end{array}$ & 4.516 & 8.000 & 24.000 & 0.002 & 0.002 & & \\
\hline
\end{tabular}

B

\begin{tabular}{|l|l|l|l|l|l|l|c|}
\hline Test Type & $\begin{array}{l}\text { Test } \\
\text { Statistic }\end{array}$ & df1 & df2 & $\begin{array}{l}\text { p- } \\
\text { value }\end{array}$ & $\begin{array}{l}\text { Permutation p- } \\
\text { value }\end{array}$ & $\begin{array}{l}\text { Global } \\
\text { Hypothesis } \\
\text { significant? }\end{array}$ & $\begin{array}{l}\text { Subset } \\
\text { Algorithm } \\
\text { continue? }\end{array}$ \\
\hline ANOVA & 7.253 & 3.758 & 11.722 & 0.004 & 0.010 & Yes & Yes \\
\hline LH Test & 2.971 & 8.000 & 15.000 & 0.033 & 0.028 & & \\
\hline BNP Test & 2.079 & 9.434 & 28.9577 & 0.063 & 0.045 & & \\
\cline { 1 - 1 } $\begin{array}{l}\text { Wilks } \\
\text { Lambda }\end{array}$ & 2.536 & 8.000 & 24.000 & 0.037 & 0.032 & & \\
\hline
\end{tabular}

C

\begin{tabular}{|l|l|l|l|l|l|l|c|}
\hline Test Type & $\begin{array}{l}\text { Test } \\
\text { Statistic }\end{array}$ & df1 & $\mathbf{d f 2}$ & $\begin{array}{l}\text { p- } \\
\text { value }\end{array}$ & $\begin{array}{l}\text { Permutation p- } \\
\text { value }\end{array}$ & $\begin{array}{l}\text { Global } \\
\text { Hypothesis } \\
\text { significant? }\end{array}$ & $\begin{array}{l}\text { Subset } \\
\text { Algorithm } \\
\text { continue? }\end{array}$ \\
\hline ANOVA & 13.240 & 4.041 & 23.3825 & 0.000 & 0.000 & Yes & Yes \\
\hline LH Test & 18.143 & 8.000 & 15.000 & 0.000 & 0.000 & & \\
\hline BNP Test & 6.175 & 9.434 & 28.9577 & 0.000 & 0.000 & & \\
\cline { 1 - 1 } $\begin{array}{l}\text { Wilks } \\
\text { Lambda }\end{array}$ & 11.057 & 8.000 & 24.000 & 0.000 & 0.000 & & \\
\hline
\end{tabular}

D

\begin{tabular}{|c|c|c|c|c|c|c|c|}
\hline Test Type & $\begin{array}{l}\text { Test } \\
\text { Statistic }\end{array}$ & df1 & df2 & $\begin{array}{l}\text { p- } \\
\text { value }\end{array}$ & $\begin{array}{l}\text { Permutation p- } \\
\text { value }\end{array}$ & $\begin{array}{l}\text { Global } \\
\text { Hypothesis } \\
\text { significant? }\end{array}$ & $\begin{array}{l}\text { Subset } \\
\text { Algorithm } \\
\text { continue? }\end{array}$ \\
\hline ANOVA & 4.931 & 5.096 & 11.6013 & 0.012 & 0.016 & \multirow[t]{4}{*}{ Yes } & \multirow[t]{4}{*}{ Yes } \\
\hline LH Test & 3.969 & 12.000 & 15.3963 & 0.006 & 0.006 & & \\
\hline BNP Test & 1.701 & 15.448 & 47.4161 & 0.082 & 0.056 & & \\
\hline $\begin{array}{l}\text { Wilks } \\
\text { Lambda }\end{array}$ & 2.678 & 12.000 & 29.3948 & 0.015 & 0.013 & & \\
\hline
\end{tabular}

E

\begin{tabular}{|c|c|c|c|c|c|c|c|}
\hline Test Type & $\begin{array}{l}\text { Test } \\
\text { Statistic }\end{array}$ & df1 & df2 & $\begin{array}{l}\text { p- } \\
\text { value }\end{array}$ & $\begin{array}{l}\text { Permutation p- } \\
\text { value }\end{array}$ & $\begin{array}{l}\text { Global } \\
\text { Hypothesis } \\
\text { significant? }\end{array}$ & $\begin{array}{l}\text { Subset } \\
\text { Algorithm } \\
\text { continue? }\end{array}$ \\
\hline ANOVA & 7.128 & 3.664 & 12,5756 & 0.004 & 0.009 & \multirow[t]{4}{*}{ Yes } & \multirow[t]{4}{*}{ Yes } \\
\hline LH Test & NA & NA & NA & NA & NA & & \\
\hline BNP Test & NA & NA & NA & NA & NA & & \\
\hline $\begin{array}{l}\text { Wilks } \\
\text { Lambda }\end{array}$ & NA & NA & NA & NA & NA & & \\
\hline
\end{tabular}




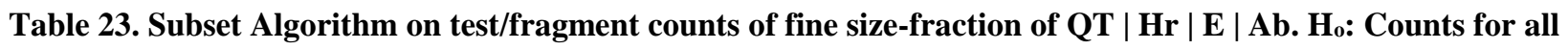
taxa are equal; $H_{a}$ : At least one taxa group is not equal.

\begin{tabular}{|c|c|c|c|c|c|c|}
\hline $\begin{array}{l}\text { Environmental } \\
\text { Parameter }\end{array}$ & QT & $\mathbf{H r}$ & $\mathbf{E}$ & $\mathbf{A b}$ & Statistical Exceptions & Color key \\
\hline \multicolumn{7}{|l|}{ Depth } \\
\hline Temperature & & & & & *At least one taxa is not equal & $\begin{array}{l}\text { Most statistically similar } \\
\text { taxa }\end{array}$ \\
\hline Salinity & & & & & & $\begin{array}{l}\text { 1st order statistically } \\
\text { distinguishable taxa }\end{array}$ \\
\hline RDO & & & & & & $\begin{array}{l}2^{\text {nd }} \text { order statistically } \\
\text { distinguishable taxa }\end{array}$ \\
\hline $\mathrm{pH}$ & & & & & & $\begin{array}{l}3^{\text {rd }} \text { order statistically } \\
\text { distinguishable taxa }\end{array}$ \\
\hline
\end{tabular}


Table 24. Subset Algorithm on depth zones of fine size-fraction of QT | Hr | E | Ab. $\mathrm{H}_{0}$ : All depth zones contributed equally to significant differences in counts. $\mathrm{H}_{\mathrm{a}}$ : At least one depth zone contributed differently to significant differences in counts

\begin{tabular}{|l|l|}
\hline Depth Zones & Color Key \\
\hline Shallow Sandy Zone $(0-10 \mathrm{~m})$ & $\begin{array}{l}\text { 1st order statistically } \\
\text { distinguishable zone }\end{array}$ \\
\hline Oculina Zone $(10-18 \mathrm{~m})$ & $\begin{array}{l}\mathbf{2}^{\text {nd }} \text { order statistically } \\
\text { distinguishable zone }\end{array}$ \\
\hline Subthermocline Zone $(18-25 \mathrm{~m})$ & *Indistinguishable \\
\hline
\end{tabular}


Table 25. Subset Algorithm on temperature zones of fine size-fraction of QT | Hr | E | Ab. Ho: All temperature zones contributed equally to significant differences in counts. $H_{a}$ : At least one temperature zone contributed differently to significant differences in counts.

\begin{tabular}{|l|l|}
\hline Temperature Zones & Color Key \\
\hline Mixed Layer (26-28 C) & \\
\hline Thermocline Layer (24-26 C) & *At least one zone is not equal \\
\hline Subthermocline Layer (22-24 C) & \\
\hline
\end{tabular}


Table 26 Subset Algorithm on salinity zones of fine size-fraction of QT | $\mathrm{Hr}|\mathrm{E}| \mathrm{Ab}$. $\mathrm{H}_{0}$ : All salinity level zones contributed equally to significant differences in counts; $H_{a}$ : At least salinity level zone contributed differently to significant differences in counts.

\begin{tabular}{|l|l|}
\hline Salinity Zones & Color Key \\
\hline Mesohaline (2-10 psu) & Most statistically similar zone \\
\hline Polyhaline (10-35 psu) & $\begin{array}{l}\text { 1st order statistically } \\
\text { distinguishable zone }\end{array}$ \\
\hline Euhaline (35+ psu) & Most statistically similar zone \\
\hline
\end{tabular}


Table 27. Subset Algorithm on dissolved oxygen zones of fine size-fraction of QT | Hr | E | Ab. $\mathrm{H}_{0}$ : All dissolved oxygen level zones contributed equally to significant differences in counts; $\mathrm{H}_{\mathrm{a}}$ : At least one dissolved oxygen level zone contributed differently to significant differences in counts.

\begin{tabular}{|l|l|}
\hline RDO Zones & \\
\hline $0-2 \mathrm{mg} / \mathrm{L}$ & \\
\hline $2-4 \mathrm{mg} / \mathrm{L}$ & \\
\hline $4-6 \mathrm{mg} / \mathrm{L}$ & *At least one zone is not equal \\
\hline $6-9 \mathrm{mg} / \mathrm{L}$ & \\
\hline
\end{tabular}


Table 28. Subset Algorithm on pH zones of fine size-fraction of QT | Hr | E | Ab. $\mathrm{H}_{0}$ : All pH intervals contributed equally to significant differences in counts; $\mathrm{H}_{\mathrm{a}}$ : At least one $\mathrm{pH}$ interval contributed differently to significant differences in counts.

\begin{tabular}{|l|l|}
\hline pH Zones & Color Key \\
\hline $8.0-8.2$ & $\begin{array}{l}\text { 1st order statistically } \\
\text { distinguishable zone } \\
\text { Most statistically similar zone }\end{array}$ \\
\hline $7.8-8.0$ & Most statistically similar zone \\
\hline $7.6-7.8$ & \\
\hline
\end{tabular}


Table 29. Relative probabilities results on environmental factors of fine size-fraction of QT | Hr |E | Ab. (A) Depth; (B) Temperature; (C) Salinity; (D) RDO; (E) pH.

A

\begin{tabular}{|l|l|l|l|}
\hline 1/8 - 1/4 mm QT spp. & $\mathbf{1 / 8}-\mathbf{1 / 4} \mathbf{~ m m}$ Hr & $\mathbf{1 / 8}-\mathbf{1 / 4} \mathbf{~ m m}$ E spp. & $\mathbf{1 / 8}-\mathbf{1 / 4} \mathbf{~ m m ~ A b}$ \\
\hline 0.833, Subthermocline & 0.815, Shallow & 0.597, Oculina Zone & 0.944, \\
Zone; 0.594, Oculina & Sandy Zone & & Subthermocline \\
Zone & & & $\begin{array}{l}\text { Zone; 0.506, } \\
\text { Oculina Zone }\end{array}$ \\
\hline
\end{tabular}

B

\begin{tabular}{|c|c|c|c|}
\hline 1/8 - 1/4 mm QT spp. & $1 / 8-1 / 4 \mathrm{~mm} \mathrm{Hr}$ & 1/8 - 1/4 mm E spp. & $1 / 8-1 / 4 \mathrm{~mm} \mathrm{Ab}$ \\
\hline $\begin{array}{l}0.833, \text { Subthermocline } \\
\text { Layer }\end{array}$ & $\begin{array}{l}0.563, \text { Thermocline } \\
\text { Layer; } 0.535, \\
\text { Mixed Layer }\end{array}$ & 0.539, Mixed Layer & $\begin{array}{l}0.944, \\
\text { Subthermocline } \\
\text { Layer; } 0.729 \text {, } \\
\text { Thermocline } \\
\text { Layer }\end{array}$ \\
\hline
\end{tabular}

C

\begin{tabular}{|l|l|l|l|}
\hline $\mathbf{1 / 8}-\mathbf{1 / 4} \mathbf{~ m m}$ QT spp. & $\mathbf{1 / 8}-\mathbf{1 / 4} \mathbf{~ m m ~ H r}$ & $\mathbf{1 / 8}-\mathbf{1 / 4} \mathbf{~ m m ~ E}$ spp. & $\mathbf{1 / 8}-\mathbf{1 / 4} \mathbf{~ m m ~ A b}$ \\
\hline 0.764, Polyhaline Zone; & 0.714, Mesohaline & 0.889, Polyhaline & 0.799, Euhaline \\
0.736, Euhaline Zone & Zone & Zone & Zone \\
\hline
\end{tabular}

D

\begin{tabular}{|l|l|l|l|}
\hline $\mathbf{1 / 8}-\mathbf{1 / 4} \mathbf{~ m m}$ QT spp. & $\mathbf{1 / 8}-\mathbf{1 / 4} \mathbf{~ m m ~ H r}$ & $\mathbf{1 / 8}-\mathbf{1 / 4} \mathbf{~ m m ~ E} \mathbf{~ s p p . ~}$ & $\mathbf{1 / 8}-\mathbf{1 / 4} \mathbf{~ m m ~} \mathbf{A b}$ \\
\hline $0.833,0-2 \mathrm{mg} / \mathrm{L} ; 0.662$, & $0.681,6-9 \mathrm{mg} / \mathrm{L} ;$ & $0.579,4-6 \mathrm{mg} / \mathrm{L}$ & $0.944,0-2 \mathrm{mg} / \mathrm{L} ;$ \\
$4-6 \mathrm{mg} / \mathrm{L}$ & $0.528,2-4 \mathrm{mg} / \mathrm{L}$ & & $0.806,2-4 \mathrm{mg} / \mathrm{L} ;$ \\
& & & $0.537,4-6 \mathrm{mg} / \mathrm{L}$ \\
\hline
\end{tabular}

E

\begin{tabular}{|l|l|l|l|}
\hline 1/8 - 1/4 mm QT spp. & $\mathbf{1 / 8}-\mathbf{1 / 4} \mathbf{~ m m ~ H r}$ & $\mathbf{1 / 8}-\mathbf{1 / 4} \mathbf{~ m m}$ E spp. & $\mathbf{1 / 8}-\mathbf{1 / 4} \mathbf{~ m m ~ A b}$ \\
\hline $0.833,7.6-7.8$ & $0.556,8.0-8.2 ;$ & $0.567,8.0-8.2$ & $0.944,7.6-7.8 ;$ \\
& $0.519,7.8-8.0$ & & $0.704,7.8-8.0$ \\
\hline
\end{tabular}


Table 30. Global Hypothesis ANOVA tests of very fine test/fragment-size fraction of QT | Hr | E | Ab

Environmental factors. $H_{0}$ : All factor levels have same effect on dead test and fragment counts; $H_{a}$ : At least one factor level has different effect on dead test and fragment counts. (A) Depth; (B) Temperature; (C)

A

Salinity; (D) RDO; (E) pH

\begin{tabular}{|c|c|c|c|c|c|c|c|}
\hline Test Type & $\begin{array}{l}\text { Test } \\
\text { Statistic }\end{array}$ & df1 & df 2 & $\begin{array}{l}\text { p- } \\
\text { value }\end{array}$ & $\begin{array}{l}\text { Permutation p- } \\
\text { value }\end{array}$ & $\begin{array}{l}\text { Global } \\
\text { Hypothesis } \\
\text { significant? }\end{array}$ & $\begin{array}{l}\text { Subset } \\
\text { Algorithm } \\
\text { continue? }\end{array}$ \\
\hline ANOVA & 5.980 & 3.910 & 13.4216 & 0.006 & 0.017 & \multirow[t]{4}{*}{ Yes } & \multirow[t]{4}{*}{ Yes } \\
\hline LH Test & 6.952 & 8.000 & 15.000 & 0.001 & 0.001 & & \\
\hline BNP Test & 4.930 & 9.434 & 28.9577 & 0.000 & 0.001 & & \\
\hline $\begin{array}{l}\text { Wilks } \\
\text { Lambda }\end{array}$ & 5.943 & 8.000 & 24.000 & 0.000 & 0.001 & & \\
\hline
\end{tabular}

B

\begin{tabular}{|l|l|l|l|l|l|c|c|}
\hline Test Type & $\begin{array}{l}\text { Test } \\
\text { Statistic }\end{array}$ & df1 & df2 & $\begin{array}{l}\text { p- } \\
\text { value }\end{array}$ & $\begin{array}{l}\text { Permutation p- } \\
\text { value }\end{array}$ & $\begin{array}{l}\text { Global } \\
\text { Hypothesis } \\
\text { significant? }\end{array}$ & $\begin{array}{l}\text { Subset } \\
\text { Algorithm } \\
\text { continue? }\end{array}$ \\
\hline ANOVA & NA & NA & NA & NA & NA & No & No \\
\hline LH Test & NA & NA & NA & NA & NA & & \\
\hline BNP Test & NA & NA & NA & NA & NA & & \\
\hline $\begin{array}{l}\text { Wilks } \\
\text { Lambda }\end{array}$ & NA & NA & NA & NA & NA & & \\
\hline
\end{tabular}

C

\begin{tabular}{|l|l|l|l|l|l|l|c|}
\hline Test Type & $\begin{array}{l}\text { Test } \\
\text { Statistic }\end{array}$ & df1 & df2 & $\begin{array}{l}\text { p- } \\
\text { value }\end{array}$ & $\begin{array}{l}\text { Permutation p- } \\
\text { value }\end{array}$ & $\begin{array}{l}\text { Global } \\
\text { Hypothesis } \\
\text { significant? }\end{array}$ & $\begin{array}{l}\text { Subset } \\
\text { Algorithm } \\
\text { continue? }\end{array}$ \\
\hline ANOVA & 8.891 & 5.070 & 29.3361 & 0.000 & 0.000 & Yes & Yes \\
\hline LH Test & 7.201 & 8.000 & 15.000 & 0.001 & 0.001 & \\
\hline BNP Test & 5.150 & 9.434 & 28.9577 & 0.000 & 0.001 & & \\
\cline { 1 - 1 } $\begin{array}{l}\text { Wilks } \\
\text { Lambda }\end{array}$ & 6.180 & 8.000 & 24.000 & 0.000 & 0.001 & & \\
\hline
\end{tabular}

D

\begin{tabular}{|l|l|l|l|l|l|c|c|}
\hline Test Type & $\begin{array}{l}\text { Test } \\
\text { Statistic }\end{array}$ & df1 & df2 & $\begin{array}{l}\text { p- } \\
\text { value }\end{array}$ & $\begin{array}{l}\text { Permutation p- } \\
\text { value }\end{array}$ & $\begin{array}{l}\text { Global } \\
\text { Hypothesis } \\
\text { significant? }\end{array}$ & $\begin{array}{l}\text { Subset } \\
\text { Algorithm } \\
\text { continue? }\end{array}$ \\
\hline ANOVA & NA & NA & NA & NA & NA & No & No \\
\hline LH Test & NA & NA & NA & NA & NA & & \\
\hline BNP Test & NA & NA & NA & NA & NA & & \\
\cline { 1 - 1 } & $\begin{array}{l}\text { Wilks } \\
\text { Lambda }\end{array}$ & NA & NA & NA & NA & NA & \\
\hline
\end{tabular}

E

\begin{tabular}{|l|l|l|l|l|l|l|c|}
\hline Test Type & $\begin{array}{l}\text { Test } \\
\text { Statistic }\end{array}$ & df1 & df2 & $\begin{array}{l}\text { p- } \\
\text { value }\end{array}$ & $\begin{array}{l}\text { Permutation p- } \\
\text { value }\end{array}$ & $\begin{array}{l}\text { Global } \\
\text { Hypothesis } \\
\text { significant? }\end{array}$ & $\begin{array}{l}\text { Subset } \\
\text { Algorithm } \\
\text { continue? }\end{array}$ \\
\hline ANOVA & 3.109 & 3.262 & 11.1942 & 0.067 & 0.089 & Yes & Yes \\
\hline LH Test & 2.637 & 8.000 & 15.000 & 0.050 & 0.053 & & \\
\hline BNP Test & 2.283 & 9.434 & 28.9577 & 0.043 & 0.036 & & \\
\cline { 1 - 1 } $\begin{array}{l}\text { Wilks } \\
\text { Lambda }\end{array}$ & 2.481 & 8.000 & 24.000 & 0.041 & 0.045 & & \\
\hline
\end{tabular}


Table 31. Subset Algorithm on test/fragment counts of very fine size-fraction of QT $|\mathrm{Hr}| \mathrm{E} \mid \mathrm{Ab}$. $\mathrm{H}_{0}$ : Counts for all taxa are equal; $H_{a}$ : At least one taxa group is not equal.

\begin{tabular}{|l|l|l|l|l|l|l|}
\hline $\begin{array}{l}\text { Environmental } \\
\text { Parameter }\end{array}$ & QT & Hr & E & Ab & Statistical Exceptions & Color key \\
\hline Depth & & & & & & \\
\hline Temperature & & & & & *Not significant & Most statistically similar taxa \\
\hline Salinity & & & & & & $\begin{array}{l}\text { 1st order statistically } \\
\text { distinguishable taxa }\end{array}$ \\
\hline RDO & & & & & *Not significant & $\begin{array}{l}2^{\text {nd }} \text { order statistically } \\
\text { distinguishable taxa }\end{array}$ \\
\hline pH & & & & & *At least one taxa is not equal & \\
\hline
\end{tabular}


Table 32. Subset Algorithm on depth zones of very fine size-fraction of QT | Hr | E | Ab. $\mathrm{H}_{0}$ : All depth zones contributed equally to significant differences in counts. $H_{a}$ : At least one depth zone contributed differently to significant differences in counts

\begin{tabular}{|l|l|}
\hline Depth Zones & Color Key \\
\hline Shallow Sandy Zone $(0-10 \mathrm{~m})$ & $\begin{array}{l}\text { 1st order statistically } \\
\text { distinguishable zone }\end{array}$ \\
\hline Oculina Zone $(10-18 \mathrm{~m})$ & $\begin{array}{l}\text { 2md order statistically } \\
\text { distinguishable zone }\end{array}$ \\
\hline Subthermocline Zone $(18-25 \mathrm{~m})$ & *Indistinguishable \\
\hline
\end{tabular}


Table 33. Subset Algorithm on temperature zones of very fine size-fraction of QT | Hr | E | Ab. $\mathrm{H}_{0}$ : All temperature zones contributed equally to significant differences in counts. $\mathrm{H}_{\mathrm{a}}$ : At least one temperature zone contributed differently to significant differences in counts.

\begin{tabular}{|l|l|}
\hline Temperature Zones & \\
\hline Mixed Layer (26-28 C) & \\
\hline Thermocline Layer (24-26 C) & *Not Significant \\
\hline Subthermocline Layer (22-24 C) & \\
\hline
\end{tabular}


Table 34. Subset Algorithm on salinity zones of very fine size-fraction of QT | Hr | E | Ab. $\mathrm{H}_{0}$ : All salinity level zones contributed equally to significant differences in counts; $H_{a}$ : At least salinity level zone contributed differently to significant differences in counts.

\begin{tabular}{|l|l|}
\hline Salinity Zones & Color Key \\
\hline Mesohaline (2-10 psu) & $\begin{array}{l}\text { 1st order statistically } \\
\text { distinguishable zone }\end{array}$ \\
\hline Polyhaline (10-35 psu) & $\begin{array}{l}\text { distinguishable zone } \\
\text { disticaly }\end{array}$ \\
\hline Euhaline (35+ psu) & *Indistinguishable \\
\hline
\end{tabular}


Table 35. Subset Algorithm on dissolved oxygen zones very fine size-fraction of QT | Hr | E | Ab. $\mathrm{H}_{0}$ : All dissolved oxygen level zones contributed equally to significant differences in counts; $H_{a}$ : At least one dissolved oxygen level zone contributed differently to significant differences in counts.

\begin{tabular}{|l|l|}
\hline RDO Zones & \\
\hline $0-2 \mathrm{mg} / \mathrm{L}$ & \\
\hline $2-4 \mathrm{mg} / \mathrm{L}$ & \\
\hline $4-6 \mathrm{mg} / \mathrm{L}$ & *Not Significant \\
\hline $6-9 \mathrm{mg} / \mathrm{L}$ & \\
\hline
\end{tabular}


Table 36. Subset Algorithm on $\mathrm{pH}$ zones of very fine size-fraction of QT $|\mathrm{Hr}| \mathrm{E} \mid \mathrm{Ab}$. $\mathrm{H}_{\mathbf{0}}$ : All pH intervals contributed equally to significant differences in counts; $\mathrm{H}_{\mathrm{a}}$ : At least one $\mathrm{pH}$ interval contributed differently to significant differences in counts.

\begin{tabular}{|l|l|}
\hline pH Zones & \\
\hline $8.0-8.2$ & \\
\hline $7.8-8.0$ & *At least one zone is not equal \\
\hline $7.6-7.8$ & \\
\hline
\end{tabular}


Table 37. Relative probabilities results on environmental factors of 0 very fine size-fraction of QT | Hr | E | Ab. (A) Depth; (B) Salinity; (C) pH.

A

\begin{tabular}{|l|l|l|l|}
\hline 1/16 - 1/8 mm QT spp. & $\mathbf{1 / 1 6}-\mathbf{1 / 8} \mathbf{~ m m ~ H r}$ & $\mathbf{1 / 1 6}-\mathbf{1 / 8} \mathbf{~ m m}$ E spp. & $\mathbf{1 / 1 6}-\mathbf{1 / 8} \mathbf{~ m m}$ Ab \\
\hline 0.636, Oculina Zone & 0.875, & 0.669, Oculina Zone; & 0.824, Shallow \\
& Subthermocline & 0.597, Subthermocline & Sandy Zone \\
& $\begin{array}{l}\text { Zone; 0.597, } \\
\text { Shallow Sandy } \\
\text { Zone }\end{array}$ & & \\
\hline
\end{tabular}

B

\begin{tabular}{|l|l|l|l|}
\hline $\mathbf{1 / 1 6}-\mathbf{1 / 8} \mathbf{~ m m}$ QT spp. & $\mathbf{1 / 1 6}-\mathbf{1 / 8} \mathbf{~ m m} \mathbf{H r}$ & $\mathbf{1 / 1 6}-\mathbf{1 / 8} \mathbf{~ m m}$ E spp. & $\mathbf{1 / 1 6}-\mathbf{1 / 8} \mathbf{~ m m}$ Ab \\
\hline 0.875, Polyhaline Zone & 0.799, Euhaline & 0.847, Polyhaline & 0.636, Mesohaline \\
& $\begin{array}{l}\text { Zone; } 0.536, \\
\text { Polyhaline Zone }\end{array}$ & $\begin{array}{l}\text { Zone; 0.535, Euhaline } \\
\text { Zone }\end{array}$ & Zone \\
\hline
\end{tabular}

\begin{tabular}{|l|l|l|l|}
\hline $\mathbf{1 / 1 6}-\mathbf{1 / 8} \mathbf{~ m m}$ QT spp. & $\mathbf{1 / 1 6}-\mathbf{1 / 8} \mathbf{~ m m} \mathbf{~ H r}$ & $\mathbf{1 / 1 6}-\mathbf{1 / 8} \mathbf{~ m m}$ E spp. & $\mathbf{1 / 1 6}-\mathbf{1 / 8} \mathbf{~ m m} \mathbf{A b}$ \\
\hline $0.592,8.0-8.2$ & $0.875,7.6-7.8 ;$ & $0.597,7.6-7.8 ; 0.551$, & $0.633,8.0-8.2$ \\
& $0.537,7.8-8.0$ & $7.8-8.0$ & \\
\hline
\end{tabular}


Table 38. Stress-value calculation after nMDS procedure.

\begin{tabular}{|l|c|l|l|}
\hline Distance & Dimensions & Stress & Stress Type 1 \\
\hline $\begin{array}{l}\text { Bray } \\
\text { Distance }\end{array}$ & 2 & 0.017 & $\begin{array}{l}\text { Two convergent } \\
\text { solutions after 20 } \\
\text { trials }\end{array}$ \\
\hline
\end{tabular}

